\title{
Continuous Wavelet \\ Transformation on Homogeneous Spaces
}

\author{
Dissertation \\ zur Erlangung des mathematisch-naturwissenschaftlichen Doktorgrades \\ "Doctor rerum naturalium" \\ der Georg-August-Universität zu Göttingen \\ im Promotionsprogramm \\ "Mathematical Sciences (Ph.D)" \\ der Georg-August University School of Science (GAUSS) \\ vorgelegt von \\ Burkhard Blobel \\ aus Freiberg/Sa.
}

Göttingen, 2020 


\section{Betreuungsausschuss:}

Prof. Dr. Dorothea Bahns,

Mathematisches Institut, Universität Göttingen

Prof. Dr. Thomas Schick,

Mathematisches Institut, Universität Göttingen

Mitglieder der Prüfungskommission:

Referent:

Prof. Dr. Dorothea Bahns,

Mathematisches Institut, Universität Göttingen

Korreferent:

Prof. Dr. Thomas Schick,

Mathematisches Institut, Universität Göttingen

\section{Weitere Mitglieder der Prüfungskommission:}

Jun.-Prof. Dr. Madeleine Jotz Lean

Mathematisches Institut, Universität Göttingen

Prof. Dr. Ralf Meyer,

Mathematisches Institut, Universität Göttingen

Prof. Dr. Gerlind Plonka-Hoch,

Institut für Numerische und Angewandte Mathematik, Universität Göttingen

Prof. Dr. Dominic Schuhmacher

Institut für Mathematische Stochastik, Universität Göttingen

Tag der mündlichen Prüfung: 11.12.2020 


\section{Contents}

$\begin{array}{ll}\text { Notation and convention } & \mathbf{v}\end{array}$

\begin{tabular}{lll}
\hline 1 & Introduction & 1
\end{tabular}

\begin{tabular}{llr}
\hline 2 & Basics & 7
\end{tabular}

2.1 Locally compact groups . . . . . . . . . . . . . . . . . . . . . 7

2.1 .1 Topological groups $\ldots \ldots \ldots \ldots$. . . . . . . . . . . . 7

2.1 .2 Homogeneous spaces of locally compact groups . . . . . . . . . 9

$2.1 .3 \quad$ Measures on locally compact spaces . . . . . . . . . . . . 10

2.1 .4 Haar measure . . . . . . . . . . . . . . . . . . . . . . . 13

2.1 .5 Function spaces $\ldots \ldots \ldots \ldots \ldots$

$2.1 .6 \quad$ Quasi-invariant measures on the quotient space . . . . . . . . . 17

$2.1 .7 \quad$ Standard Borel spaces . . . . . . . . . . . . . . . . . 22

2.2 Representation theory $\ldots \ldots \ldots \ldots \ldots$. . . . . . . . . . . 27

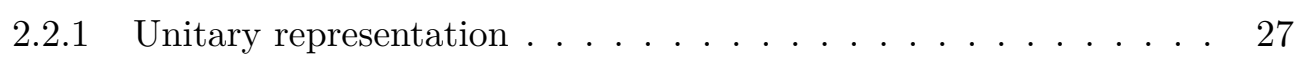

2.2 .2 Induced representations $\ldots \ldots \ldots \ldots$

$2.2 .3 \quad$ Properties of induced representations. . . . . . . . . . . . . . 34

2.3 The dual and the quasi-dual of a locally compact group . . . . . . . . 35

$2.3 .1 \quad$ Von Neumann algebras $\ldots \ldots \ldots \ldots$

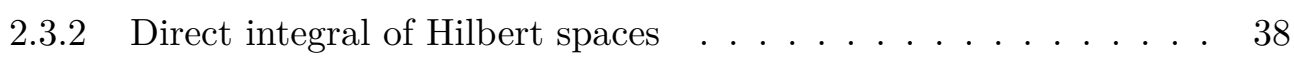

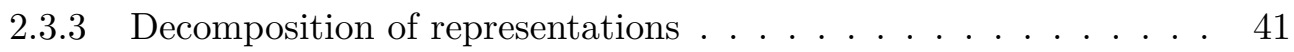

$2.3 .4 \quad$ Plancherel decomposition . . . . . . . . . . . . . . . . . . 44

2.3 .5 Remarks on the Plancherel Theorem . . . . . . . . . . . . . . 47

2.4 Contractions of Lie algebras, Lie groups, and representation . . . . . . . 48

\begin{tabular}{|lll}
3 & Continuous wavelet transformations and admissibility conditions & 51
\end{tabular}

3.1 Continuous wavelet transformations and generalizations . . . . . . . . . 51

3.1 .1 Classical continuous wavelet transformation . . . . . . . . . . . . 51

$3.1 .2 \quad$ Shearlets and semidirect products . . . . . . . . . . . . 55

3.1.3 Windowed Fourier transformation and coherent state transformation . . . . . . . . . . . . . . . . 57

3.1 .4 Coherent states over coadjoint orbits . . . . . . . . . . . . . . 59

3.1 .5 Continuous wavelet transformations on manifolds . . . . . . . . 60 
3.1 .6 Continuous diffusion wavelet transformations . . . . . . . . . . 63

3.2 Group-theoretical approach to continuous wavelet transformations . . . 64

$3.2 .1 \quad$ Introduction and definitions . . . . . . . . . . . . . 65

3.2 .2 Square-integrability condition . . . . . . . . . . . . . 67

$\begin{array}{|lll|}4 & \text { New approaches to continuous wavelet transformations } & \mathbf{7 5}\end{array}$

4.1 Relation between representations $\ldots \ldots \ldots$. . . . . . . . . 76

4.2 Continuous wavelet transformations on unimodular groups. . . . . . . . 80

4.3 Continuous wavelet transformations on homogeneous spaces . . . . . . . 81

$4.3 .1 \quad$ Semidirect products $\ldots \ldots \ldots \ldots \ldots$. . . . . . . . . . . 82

$4.3 .2 \quad$ Examples $\ldots \ldots \ldots \ldots \ldots$

$4.3 .3 \quad$ Group extensions . . . . . . . . . . . . . . . . . . . . . . . . . . 97

4.4 Continuous wavelet transformations on manifolds . . . . . . . . . . . . . 101

$\begin{array}{lll}5 & \text { Outlook and discussion } & 105\end{array}$

\begin{tabular}{|ll|}
\hline A Polar decomposition of direct integral operators & 109
\end{tabular}

\begin{tabular}{|l|l|}
\hline B Fourier transformation of tempered distributions & 113
\end{tabular} 


\section{Notation and convention}

\section{Notation}

$\begin{array}{ll}\operatorname{im} \varphi & \text { image of a map } \varphi \\ \operatorname{ker} \varphi & \text { kernel of a homomorphism } \varphi \\ A^{t} & \text { transpose of a matrix } A \\ A^{-t} & \text { inverse transposed matrix } \\ \operatorname{char}_{E} & \text { characteristic function of a measurable set } E \\ \text { ess sup } E & \text { essential supremum of a measurable set, } \\ & \text { ess sup } E=\text { inf }\{b \in \mathbb{R} \mid\{x \in E \mid x>b\} \text { is a null set }\} \\ C_{c}(X) & \text { space of compactly supported continuous functions } \\ C_{0}(X) & \text { space of continuous functions vanishing at infinity } \\ C_{b}(X) & \text { space of bounded continuous functions } \\ \|\cdot\|_{\infty} & \text { uniform norm (supremum norm) } \\ \mathbb{1} \in \mathcal{H} & \text { identity operator on a Hilbert space } \mathcal{H} \\ \mathcal{B}\left(V_{1} ; V_{2}\right) & \text { space of bounded linear operators from a Banach space } V_{1} \\ & \text { to a Banach space } V_{2} \\ \mathcal{B}(\mathcal{H})=\mathcal{B}(\mathcal{H} ; \mathcal{H}) & \text { space of bounded linear operators on a Hilbert space } \mathcal{H} \\ & \text { (defined via functional calculus) } \\ \mathcal{B}_{1}(\mathcal{H}) & \text { space of trace-class operators on a Hilbert space } \mathcal{H} \\ \mathcal{B} \mathcal{B}_{2}(\mathcal{H}) & \text { space of Hilbert-Schmidt operators on a Hilbert space } \mathcal{H} \\ \mathcal{U}(\mathcal{H}) & \text { absolute value of an operator } T: \mathcal{H} \rightarrow \mathcal{H}, \\ \|T\|_{1} & \text { trace norm of an operator } T: \mathcal{H} \rightarrow \mathcal{H} \\ \|T\|_{2} & \end{array}$


$\Delta_{G} \quad$ modular function of a locally compact group $G$

$\lambda_{G} \quad$ left regular representation of a locally compact group $G$

$\mu_{G} \quad$ Plancherel measure of a locally compact group $G$

$\mathbb{N}_{0}=\{0,1,2, \cdots\} \quad$ natural numbers including 0

$\mathbb{N}=\{1,2,3, \cdots\} \quad$ natural numbers without 0

$\overline{\mathbb{N}}=\mathbb{N} \cup\{\infty\} \quad$ countable cardinalities without 0

\section{Convention}

$$
\begin{array}{ll}
\int_{\mathbb{R}^{n}} e^{2 \pi i\langle k, x\rangle} f(x) \mathrm{d} x & \begin{array}{l}
\text { Fourier transform of a function } f \in L^{1}\left(\mathbb{R}^{n}\right), \\
\end{array} \\
& \text { Plancherel transform of a function } f \in L^{1}\left(\mathbb{R}^{n}\right) \cap L^{2}\left(\mathbb{R}^{n}\right) \\
\langle\cdot, \cdot\rangle & \text { (complex) scalar product, } \\
& \text { antilinear in the first argument, linear in the second argument } \\
\int_{G} f(a) \mathrm{d} a & \text { integration against the left Haar measure } \\
& \text { of a locally compact group } G \\
H \leq G & H \text { is closed subgroup of a locally compact group } G
\end{array}
$$

\section{Terminology}

- neighborhood of a point: a subset of a topological space containing the point in its interior (not necessarily open)

- action: left action

- representation: strongly continuous unitary representation 


\section{CHAPTER 1}

\section{Introduction}

Wavelet transformation is a tool coming from data analysis. Roughly speaking, it has its origin in analyzing seismic measurements in geophysics and goes back to Goupillaud, Grossmann, and Morlet. In [38], they discussed the problem of reconstructing and resolving underground structures in order to find reservoirs of oil and gas. The data used for that purpose consists of a superposition of seismic waves, which are backscattered by the different layers in the ground and are measured over time. Since information about the thicknesses and impedances of different layers are encoded in frequencies and timings, it is important to keep track of both. The straightforward approach uses windowed or short-time Fourier analysis, which means that the measured signal is decomposed into "elementary wavelets" of the form

$$
\psi_{b, a}(t)=e^{i t / a} \psi(t-b)
$$

where $\psi$ is a window function, which is typically chosen to be a Gaussian of width $T$,

$$
\psi(t)=\frac{1}{\sqrt{2 \pi T^{2}}} e^{-\frac{t^{2}}{2 T^{2}}} .
$$

This method goes back to Gabor [36]. Goupillaud, Grossmann, and Morlet demonstrate that because of the fixed width of the window, the timing resolution for high frequencies drops and results in a loss of information. Instead, they propose to use a family of waves which scale not only in frequency but also in timing. They decompose the signal into wavelets of the form

$$
\phi_{b, a}(t)=|a|^{-\frac{1}{2}} \phi\left(a^{-1}(t-b)\right)
$$

where $\phi$ can be chosen, for example, as

$$
\phi(t)=\sqrt{2} e^{-\frac{t^{2}}{T^{2}}}-e^{-\frac{t^{2}}{2 T^{2}}} .
$$

The different shapes are illustrated in Fig. 1.1. With this approach Goupillaud, Grossmann, and Morlet managed to improve the quality and resolution of the results.

To evaluate the measured signal $f$, one has to transform it into a function $V_{\phi} f(b, a)$ 


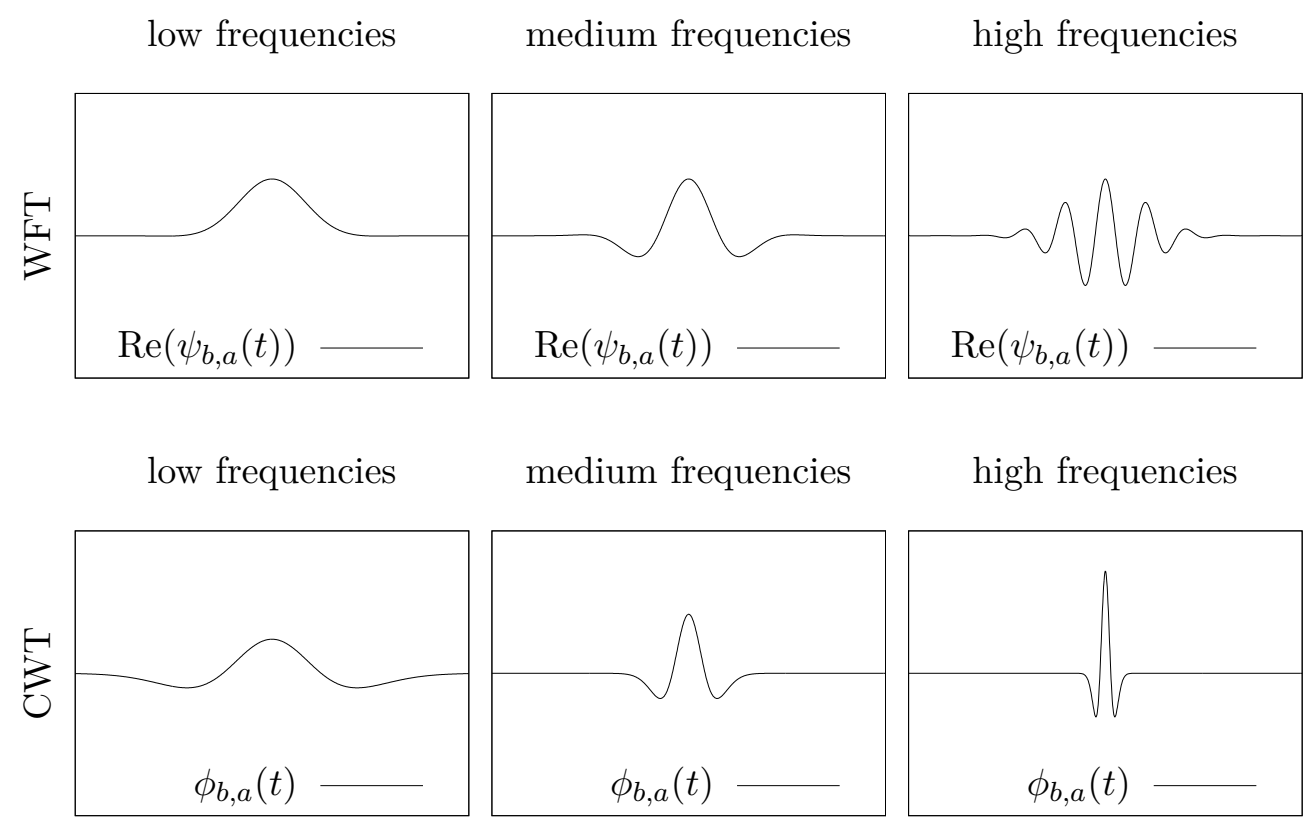

Figure 1.1: Comparison of wavelets used for windowed Fourier transformation (WFT) and continuous wavelet transformation (CWT) at low $(a=3)$, medium $(a=1)$, and high frequencies $(a=1 / 3) . \psi_{b, a}$ is defined in eq. (1.1) and eq. (1.2) with $T=2 . \phi_{b, a}$ is defined in eq. 1.3 and eq. (1.4) with $T=1$.

such that

$$
f(t)=\int_{\mathbb{R}_{\neq 0}} \int_{\mathbb{R}} V_{\phi} f(b, a) \cdot \phi_{b, a}(t) \frac{1}{a^{2}} \mathrm{~d} b \mathrm{~d} a
$$

stating that $f$ is a superposition of the functions $\left\{\phi_{b, a}\right\}$. In order to do that they noted that for suitable $\phi$ the operator $V_{\phi}: L^{2}(\mathbb{R}) \rightarrow L^{2}\left(\mathbb{R} \times \mathbb{R}_{\neq 0}, \frac{1}{a^{2}} \mathrm{~d} b \mathrm{~d} a\right)$,

$$
V_{\phi} f(b, a)=\int_{\mathbb{R}} \overline{\phi_{b, a}(t)} f(t) \mathrm{d} t
$$

is an isometry. Hence, its adjoint operator $V_{\phi}^{*}: L^{2}\left(\mathbb{R} \times \mathbb{R}_{\neq 0}, \frac{1}{a^{2}} \mathrm{~d} b \mathrm{~d} a\right) \rightarrow L^{2}(\mathbb{R})$,

$$
V_{\phi}^{*} F(t)=\int_{\mathbb{R}_{\neq 0}} \int_{\mathbb{R}} F(b, a) \cdot \phi_{b, a}(t) \frac{1}{a^{2}} \mathrm{~d} b \mathrm{~d} a,
$$

has the desired property

$$
f=V_{\phi}^{*} V_{\phi} f
$$

and $V_{\phi}$ defined in eq. (1.6) fulfills the relation in eq. (1.5). Goupillaud, Grossmann, and Morlet called the operator $V_{\phi}$ the voice transformation. Today, it is known as (classical) continuous wavelet transformation.

In [39, Grossmann, Morlet, and Paul showed that it is no coincidence that $V_{\phi}$ is 
an isometry. They stated that the map $\pi(b, a): \phi \mapsto \phi_{b, a}$ is an irreducible unitary representation of the affine group of the real line

$$
G_{\text {aff }}(\mathbb{R})=\mathbb{R} \rtimes \mathbb{R}_{\neq 0}=\left\{(b, a) \mid a \in \mathbb{R}_{\neq 0}, b \in \mathbb{R}\right\}
$$

with the group law $(b, a) \cdot\left(b^{\prime}, a^{\prime}\right)=\left(a b^{\prime}+b, a a^{\prime}\right)$. Because of its action $(b, a) \cdot x=a x+b$, for $x \in \mathbb{R}, G_{\text {aff }}(\mathbb{R})$ is also known as the $a x+b$-group. It turns out that the operator $V_{\phi}$ is an intertwiner $L^{2}(\mathbb{R}) \rightarrow L^{2}\left(G_{\text {aff }}(\mathbb{R})\right)$ between $\pi$ and the left regular representation of $G_{\text {aff }}(\mathbb{R})$. The measure $\frac{1}{a^{2}} \mathrm{~d} b \mathrm{~d} a$ is nothing but the Haar measure of $G_{\text {aff }}(\mathbb{R})$.

This observation opened the field to pure mathematics including representation theory of locally compact groups and abstract harmonic analysis. During the last decades the idea developed and led to the following definition (cf. Führ [33, Chp. 2.3]).

Definition 1.1. Let $G$ be a locally compact group and $\pi$ a (strongly continuous) unitary representation on a complex Hilbert space $\mathcal{H}$. Let $\psi \in \mathcal{H}$ be a fixed, nonzero vector.

(i) Denote by $V_{\psi}$ the possibly unbounded (not even densely defined) operator $\mathcal{H} \rightarrow$ $L^{2}(G)$ given by $V_{\psi} f(a)=\langle\pi(a) \psi, f\rangle$.

(ii) If $V_{\psi}$ is an isometry, then it is called a (generalized) continuous wavelet transform. In that case $\psi$ is called an admissible vector.

Here, we use the physics convention for the scaler product. That is, $\langle\cdot, \cdot\rangle$ is anti-linear in the first and linear in the second argument. Questions which arise naturally from the definition, are the following.

- Under which conditions does a given representation $\pi$ have admissible vectors?

- How can admissible vectors be identified?

Among other things, those are the questions Führ discusses in [33. As a first result the author states that the continuous wavelet transformation is an intertwiner between $\pi$ and the left regular representation $\lambda_{G}$ of a locally compact group $G$. As a consequence of Def. 1.1, a representation $\pi$ admits a continuous wavelet transformation only if it is a subrepresentation of $\lambda_{G}$.

Proposition 1.2. Let $G$ be a locally compact group and let $\pi$ be unitary representation of $G$. If $\pi$ has an admissible vector then $\pi$ is a subrepresentation of the left regular representation of $G\left(\pi \leq \lambda_{G}\right)$.

Another useful observation is that admissible vectors of a representation can be used to construct admissible vectors of subrepresentations.

Proposition 1.3. Let $G$ be a locally compact group and let $\rho$ and $\pi$ be unitary representations of $G$ on the Hilbert spaces $\mathcal{H}_{\pi}$ and $\mathcal{H}_{\rho}$, respectively, such that there is a unitary intertwiner $T: \mathcal{H}_{\rho} \rightarrow \mathcal{H}_{\pi}$. In other words, $\rho$ is assumed to be a subrepresentation of $\pi$. If $\pi$ has an admissible vector $\psi \in \mathcal{H}_{\pi}$ then $T^{*} \psi \in \mathcal{H}_{\rho}$ is an admissible vector for $\rho$. 
Suppose for a moment that $\lambda_{G}$ has an admissible vector, hence, admits a continuous wavelet transformation. Then, Prop. 1.2 and Prop. 1.3 show that $\pi$ has admissible vectors if and only if $\pi$ is a subrepresentation of $\lambda_{G}$. In one of his main results, Führ proves that this is actually true for nonunimodular groups.

Theorem 1.4. Let $G$ be a second countable locally compact group. Suppose that $G$ is nonunimodular and type $I$.

Then the left regular representation $\lambda_{G}$ has an admissible vector. A unitary representation $\pi$ of $G$ has admissible vectors if and only if it is a subrepresentation of $\lambda_{G}$.

Using methods from abstract harmonic analysis (i.e., central decomposition of representations) one can explicitly check whether a given representation is or is not a subrepresentation of another one.

If $G$ is unimodular the situation is more complicated.

Theorem 1.5. Let $G$ be a locally compact group. Suppose that $G$ is unimodular. Then, $\lambda_{G}$ has an admissible vector if and only if $G$ is discrete.

Nevertheless, Führ gives an explicit necessary and sufficient criterion for the existence of admissible vectors for representations of unimodular groups of type I in the general case. (Details will be given in Chp. 3.2.) Again, this criterion is formulated in term of abstract harmonic analysis.

Using the criterion found by Führ one can determine whether a given representation admits a continuous wavelet transformation. Among those which do not admit a continuous wavelet transformation there are many representations which play an important role, for instance in quantum mechanics, quantum optics, and particle physics. One of the most prominent examples are the Schrödinger representations of the HeisenbergWeyl group together with the Wigner-Weyl transformation. During the last century, physicists developed methods which look very similar to the continuous wavelet transformation defined in Def. 1.1, which are known as coherent state transformations. The notion "coherent state" goes back to Glauber [37] and comes from quantum optics. Coherent state transformations can essentially be summarized as follows (cf. Führ [33, Chp. 2.2]).

Definition 1.6. Let $G$ be a locally compact group and $\pi$ a unitary representation on a Hilbert space $\mathcal{H}$. Let $(M, \mu)$ be a measure space and let $q: M \rightarrow G$ be measurable. Let $\psi \in \mathcal{H}$ be a fixed, nonzero vector.

The family $\Psi=\{\pi(q(m)) \psi\}_{m \in M}$ is called a coherent state system if for all $f \in \mathcal{H}$

$$
\int_{M}|\langle\pi(q(m)) \psi, f\rangle|^{2} \mathrm{~d} \mu(m)=\|f\|_{\mathcal{H}}^{2}
$$

In that case the operator $W_{\Psi}: \mathcal{H} \rightarrow L^{2}(M), W_{\Psi} f(m)=\langle\pi(q(m)) \psi, f\rangle$, is called a coherent state transformation. 
If $M=G, \mu$ is the left Haar measure of $G$, and $q$ is the identity map, then Def. 1.6 goes over into Def. 1.1. In that sense, the continuous wavelet transformation is an instance of coherent state transformations. Due to the additional degrees of freedom (i.e., a suitable choice of $M, \mu$, and $q$ ), it is possible to construct coherent state transformations even if a given representation does not admit a continuous wavelet transformation. However, because of this degree of freedom a systematical examination is very challenging.

It turns out that the representations of interest are usually defined on homogeneous spaces. Let $X$ be a homogeneous $G$-space and suppose that there exists a nonzero, locally finite, $G$-invariant measure $\mu$ on $X$. One can check that

$$
[\pi(a) f](x)=f\left(a^{-1} \cdot x\right) \quad \forall f \in L^{2}(X, \mu), a \in G, x \in X,
$$

defines a representation of $G$. Indeed, $\pi$ is a example of so-called induced representations. Those representations often appear when constructing all irreducible representations of a group, for instance when using Mackey's machine (see, for example, Folland [31]) or Kirillov's orbit method (see Kirillov [46]). Moreover, induced representations frequently appear in physics, where $X$ is a physical system (position space) and $G$ is the group of motions acting transitively on $X$.

The aim of this thesis is to extend the notion of continuous wavelet transformation given in Def. 1.1 and develop generalizations. In order to do that, it is important to compile the necessary conditions for the existence of admissible vectors from Führ [33]. After identifying the reasons why a given representation does not admit a continuous wavelet transformation we start to develop strategies to bypass those problems. The main focus is on representations defined on homogeneous spaces or, in other words, on induced representations. The motivation for this topic comes from physics, where the group $G$ is usually a Lie group. However, since we will make use of the differential structure only in a few cases, $G$ is often assumed to be a second countable locally compact group. In fact, in representation theory most abstract statements which are true for Lie groups also work for second countable locally compact groups and most problems occurring for second countable locally compact groups also appear in representation theory of Lie groups. The differential structure of Lie groups comes in handy when doing explicit constructions like Kirillov's orbit method.

The thesis is structured as follows. The theoretical basics which will be used later are presented in Chp. 2, This includes the main results on locally compact spaces and groups, their representation theory and the fundamentals of abstract harmonic analysis. Moreover, the idea of Lie group contractions, which will be used in Chp. 3.1 and Chp. 4.4 is sketched in Chp. 2.4

Chp. 3 gives an overview of the known facts on continuous wavelet transformations. In Chp. 3.1 we will see the constructions and generalizations which will be relevant for this thesis. The presented ideas are just a selected collection and Chp. 3.1 is far from being a complete discourse. A more extensive picture can be found in the textbook [3] 
by Ali, Antoine, and Gazeau and the references therein. The knowledge on continuous wavelet transformations developed by Führ is summarized in Chp. 3.2 .

Chp. 4 contains the main part of this thesis. As explained above unimodular and nonunimodular groups behave differently. In some sense, this discrepancy is resolved in Chp.4.2. In Chp. 4.3 we will see a generalized continuous wavelet transformation which belongs to the class of coherent state transformations. The idea presented therein is an extension and further development of de Bièvre [20]. In Chp. 4.4 the approach by Antoine and Vandergheynst [5] and [6] is discussed. We will see that this approach has some limitations one should be aware of when using it.

The results are summarized in Chp. 5 . 


\section{CHAPTER 2}

\section{Basics}

The aim of this chapter is to explain the notions and notations which will be used later. The focus is on second countable locally compact groups, including their homogeneous spaces, integration theory, and representation theory. The books [31 by Folland, 33] by Führ, 44] by Kaniuth and Taylor, and [21] by Dixmier severed as an orientation for this chapter. Many of these results can also be found in the Bourbaki series General Topology [13], [14] and Integration [15], [16].

\section{$2.1 \quad$ Locally compact groups}

This section is dedicated to locally compact groups and their homogeneous spaces. To work out why second countability is important we start with general locally compact groups and indicate what can go wrong if they are not second countable. The results and examples presented here are mostly taken from Folland [31, Chp. 2], and Kaniuth and Taylor [44, Chp. 1]. In Chp. 2.1.3 some notions about measures on locally compact spaces are clarified in order to avoid confusions. The results given in this section are taken from Rudin [56, Chp. 2] and the textbook [27] by Elstrodt served as supplemental material. In Chp. 2.1.7 we address standard Borel spaces which are briefly discussed in Folland [31] and Führ [33]. Many of the results presented there seem to be well-known, however, it is hard to find any direct references. Therefore, the statements which will be used later are deduced from the original paper [51] by Mackey.

\subsubsection{Topological groups}

A topological group is a nonempty topological space $G$ with a continuous group structure. That means that the multiplication $G \times G \rightarrow G,(a, b) \mapsto a b$ and the inversion $G \rightarrow G, a \mapsto a^{-1}$, are continuous maps. The identity element of $G$ will be denoted by $e_{G}$ or simply by $e$. Every group (in the algebraic sense) can be considered as a topological group endowed with the discrete topology. Those groups are called discrete groups. Provided that the topology is Hausdorff, it basically describes when infinite products of group elements are well-defined and converge. For instance, one can easily verify that for discrete groups an infinite product is well-defined if and only if all but finitely many factors are trivial. Some authors include the Hausdorff property 
in the definition of a topological group. However, as we will see in Cor. 2.3 below, it is always possible to pass from a topological group to a Hausdorff topological group by identifying elements which cannot be separated.

In this thesis, we follow the notions of Bourbaki [13]. A (subset of a) topological space is called quasi-compact if every open cover has a finite subcover. It is called compact if it is quasi-compact and Hausdorff. A topological space is called locally compact if it is Hausdorff and every point has a compact neighborhood. A locally compact topological group is called a locally compact group. As we will see in Chp. 2.1.3 and Chp. 2.1.4. locally compact spaces have a nice integration theory which comes in handy when studying unitary representations of locally compact groups. This is the key to abstract harmonic analysis.

Topological groups have the following properties which are well-known and often used without mentioning them.

Proposition 2.1. Let $G$ be a topological group.

(i) Inversion and left and right translations are homeomorphisms.

(ii) If $H$ is a (normal) subgroup of $G$, then its closure $\bar{H}$ is a (normal) subgroup, as well.

(iii) Every open subgroup of $G$ is closed.

(iv) If $G_{1}$ and $G_{2}$ are topological groups, then $G_{1} \times G_{2}$ is a topological group. If $G_{1}$ and $G_{2}$ are locally compact, then so is $G_{1} \times G_{2}$.

Let $G$ be a topological group and $H$ a subgroup. Then the quotient space $G / H=$ $\{a H \mid a \in G\}$ is the space of left cosets endowed with the quotient topology induced from the canonical quotient map $p: G \rightarrow G / H, a \mapsto a H$. The following proposition shows that forming quotients is a well-behaved process in the category of locally compact groups as long as the subgroup is closed.

Proposition 2.2. Let $G$ be a topological group and $H$ a subgroup.

(i) The natural action $G \times G / H \rightarrow G / H,(a, b H) \mapsto(a b) H$, is continuous.

(ii) The quotient map $p: G \rightarrow G / H$ is an open map.

(iii) $G / H$ is Hausdorff if and only if $H$ is closed.

(iv) If $H$ is closed and $G$ is locally compact, then $H$ and $G / H$ are locally compact, as well.

(v) If $H$ is normal, then $G / H$ is a topological group.

In the following we will write $H \leq G$ if $H$ is a closed subgroup and $H \unlhd G$ if $H$ is closed and normal.

A direct consequence of Prop. 2.1 and Prop. 2.2 is the following result. 
Corollary 2.3. Let $G$ be a topological group.

(i) $G$ is Hausdorff if and only if $\{e\}$ is closed.

(ii) $\overline{\{e\}}$ is a normal subgroup and $G / \overline{\{e\}}$ is Hausdorff.

Hence, the Hausdorff property can be easily obtained whenever one can work with the quotient group $G / \overline{\{e\}}$ instead.

\subsubsection{Homogeneous spaces of locally compact groups}

Let $G$ be a locally compact group and $X$ a topological space. An action of $G$ on $X$ is a continuous map $G \times X \rightarrow X,(a, x) \mapsto a x$, such that

(i) the map $X \rightarrow X, x \mapsto a x$, is a homeomorphism for all $a \in G$,

(ii) and $a_{1}\left(a_{2} x\right)=\left(a_{1} a_{2}\right) x$ for all $a_{1}, a_{2} \in G$ and $x \in X$.

If for every $x, y \in X$ there exists an element $a \in G$ such that $y=a x$, then the action is called transitive and $X$ is called a homogeneous $G$-space (or shortly a homogeneous space).

Remark 2.4. Here, the notion "action" means left action. In contrast to left actions one can define right actions which have the property that

(ii') $a_{1}\left(a_{2} x\right)=\left(a_{2} a_{1}\right) x$ for all $a_{1}, a_{2} \in G$ and $x \in X$

instead of (ii). Right actions often appear as actions on spaces of right cosets. If $H$ is a closed subgroup of a locally compact group $G$, then

$$
G \times H \backslash G \rightarrow H \backslash G, \quad(a, H b) \mapsto H b a,
$$

defines a right action.

Both, left and right actions, are in one-to-one correspondence. If $G \times X \rightarrow X,(a, x) \mapsto$ $a x$, is a right action, then $G \times X \rightarrow X,(a, x) \mapsto a^{-1} x$ is a left action.

Throughout this thesis only left actions will appear and are referred to as actions.

An important class of homogeneous spaces are quotient spaces, introduced in Chp. 2.1.1. From the algebraic point of view any homogeneous space is ( $G$-equivariant) isomorphic to a quotient space. In the category of locally compact groups the situation is more complicated.

Let $G$ be a locally compact group and $X$ a homogeneous space. For a fixed element $x \in X$ let

$$
G_{x}:=\{a \in G \mid a x=x\}
$$

be the stabilizer of $x$. By the universal property of quotient spaces the map $f_{x}: G \rightarrow$ $X, a \mapsto a x$, factors through the canonical quotient map $p_{x}: G \rightarrow G / G_{x}$, i.e., there exists a unique continuous map $F_{x}: G / G_{x} \rightarrow X$ such that the diagram 


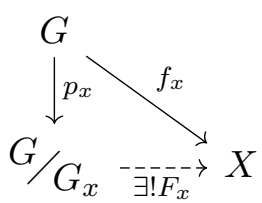

commutes. $F_{x}$ is bijective and $G$-equivariant. Since $p_{x}$ is open, $F_{x}$ is a homeomorphism if and only if $f_{x}$ is open. However, $f_{x}$ is not necessarily open as the following example shows.

Example 2.5. Let $\mathbb{R}_{\mathrm{d}}$ be the set of real numbers endowed with the discrete topology and let $\mathbb{R}$ be endowed with the standard topology (induced from its Euclidean norm). The map

$$
\mathbb{R}_{\mathrm{d}} \times \mathbb{R} \rightarrow \mathbb{R}, \quad(a, x) \mapsto a+x,
$$

is an action, which is free and transitive. The map

$$
\mathbb{R}_{\mathrm{d}} \rightarrow \mathbb{R}, \quad a \mapsto a+0,
$$

is continuous and bijective. However, it is not open and therefore not a homeomorphism.

By specifying requirements on the topology of $G$ we can get the following result.

Proposition 2.6. Let $G$ be a $\sigma$-compact locally compact group and let $X$ be a homogeneous $G$-space. Then $X$ is homeomorphic to $G / H$, where $H$ is the stabilizer of some point in $X$.

\subsubsection{Measures on locally compact spaces}

Locally compact groups, or in general all locally compact spaces, have the pleasant property that it is possible to define a nice integration theory. There are essentially two ways to define integrals. On the one hand, an integral on a measurable space (for instance a topological space endowed with the Borel $\sigma$-algebra) can be directly constructed from a measure on its $\sigma$-algebra. This procedure is standard and can be found in any introductory textbook on analysis (see Rudin [56] for example). On the other hand, every integral defines a (positive) linear functional from a vector space of functions to the complex numbers. Therefore, it seems legit to interpret a (positive) linear functional on a suitable vector space of functions (for example the space of compactly supported functions) as an integral. For locally compact spaces both approaches are closely related and enjoy several convenient properties.

Let $X$ be a topological Hausdorff space. The $\sigma$-algebra generated by its open subsets is called Borel $\sigma$-algebra. A subset of $X$ is called a Borel set if it is contained in the Borel $\sigma$-algebra. A Borel measure is a measure defined on the Borel $\sigma$-algebra. 
Definition 2.7. Let $X$ be a topological space and let $\mu$ be a Borel measure on $X$.

(i) A Borel set $E \subseteq X$ is called inner regular if

$$
\mu(E)=\sup \{\mu(K) \mid K \subseteq E, K \text { compact }\} .
$$

(ii) A Borel set $E \subseteq X$ is called outer regular if

$$
\mu(E)=\inf \{\mu(U) \mid U \supseteq E, U \text { open }\} .
$$

(iii) $\mu$ is called inner (respectively outer) regular if every Borel set is inner (respectively outer) regular. If $\mu$ is both, inner and outer regular, then $\mu$ is called regular.

(iv) $\mu$ is called inner regular on open sets if every open set is inner regular.

(v) $\mu$ is called locally finite if every point of $X$ has an open neighborhood $U$ such that $\mu(U)<\infty$.

(vi) $\mu$ is called a Radon measure if it is inner regular on open sets, outer regular, and locally finite.

(vii) $\mu$ is called $\sigma$-finite if there exists a countable family $\left(E_{n}\right)_{n \in \mathbb{N}}$ of Borel sets with $\mu\left(E_{n}\right)<\infty$ for all $n \in \mathbb{N}$ and $\bigcup_{n \in \mathbb{N}} E_{n}=X$.

This terminology is not uniformly used in all textbooks. In particular, the definition of Borel and Radon measure can differ. The notions used here are consistent with Folland [31] and Kaniuth and Taylor [44. Indeed, at least in Chp. 1 of Kaniuth and Taylor 44 there seems to be some confusion. The authors use the Riesz-MarkovKakutani Representation Theorem (see Thm. 2.9 below) to construct a regular Borel measure. However, the Riesz-Markov-Kakutani Theorem only guaranties the existence of a Radon measure, which is not necessarily regular. Anyhow, this seems be some inconsistency in notions rather than conceptional discrepancy. The aim of this section is to avoid this kind of confusion.

The properties of Borel measures depend on the topology of the underlying space. Among others they have the following features, which are direct consequences of the respective definitions.

Proposition 2.8. Let $\mu$ be a Borel measure on a topological Hausdorff space $X$.

(i) If $\mu$ is locally finite, then $\mu$ is finite on compact sets.

(ii) If $X$ is locally compact, then $\mu$ is locally finite if and only if $\mu$ is finite on compact sets.

(iii) If $X$ is $\sigma$-compact and $\mu$ is locally finite, then $\mu$ is $\sigma$-finite. 
Let $C_{c}(X)=C_{c}(X ; \mathbb{C})$ be the space of continuous, compactly supported complex-valued functions on $X$. For any Radon measure $\mu$ on $X$ the map $\lambda_{\mu}: C_{c}(X) \rightarrow \mathbb{C}$,

$$
\lambda_{\mu}(\varphi):=\int_{X} \varphi \mathrm{d} \mu
$$

defines a linear functional. $\lambda_{\mu}$ is positive, i.e., if $\varphi(x) \geq 0$ for all $x \in X$ then $\lambda_{\mu}(\varphi) \geq 0$. The Riesz-Markov-Kakutani Representation Theorem states that every positive linear functional comes from a Radon measure.

Theorem 2.9 (Riesz-Markov-Kakutani Representation Theorem).

Let $X$ be a locally compact space and let $\lambda$ be a positive linear functional. Then there exists a unique Radon measure $\mu$ on $X$ such that

$$
\lambda(f)=\int_{X} f(x) \mathrm{d} \mu(x) .
$$

If $X$ is $\sigma$-compact, then $\mu$ is regular.

The detailed proof can be found in Rudin [56, Chp. 2].

Regular Radon measures are preferable since they behave - from the topological point of view - as one would expect. For example every Borel set $E$ which is locally null is a null set. (Locally null means that for every Borel set $F \subseteq X$ of finite measure the intersection $E \cap F$ is a null set.) If $\mu$ is a regular Borel measure and $E$ is locally null then, in particular, $\mu(K)=0$ for every compact $K \subseteq E$ and hence by inner regularity it follows that $\mu(E)=0$. For nonregular Radon measures this is, in general, not true as the following example shows.

Example 2.10. Let $\mathbb{R}_{\mathrm{d}}$ be the set of real numbers endowed with discrete topology and let $\mathbb{R}$ be the real numbers endowed with standard topology. Denote the Lebesgue measure of $\mathbb{R}$ by $\mu$. Then the product space $X=\mathbb{R} \times \mathbb{R}_{\mathrm{d}}$ is locally compact as $\mathbb{R}$ and $\mathbb{R}_{\mathrm{d}}$ are. $A$ subset $A \subseteq X$ is open, closed, or measurable if and only if $A \cap(\mathbb{R} \times\{y\})$ is open, closed, or measurable for all $y \in \mathbb{R}_{\mathrm{d}} . K \subseteq X$ is compact if and only if $K \cap(\mathbb{R} \times\{y\})$ is compact for all $y \in \mathbb{R}_{\mathrm{d}}$ and there is a finite subset $I \subseteq \mathbb{R}_{\mathrm{d}}$ such that $K \subseteq \mathbb{R} \times I$. Let $\nu$ be the Radon measure on $X$ corresponding to the linear functional $\lambda: C_{c}(X) \rightarrow \mathbb{C}$

$$
\lambda(f)=\sum_{y \in \mathbb{R}_{\mathrm{d}}} \int_{\mathbb{R}} f(x, y) \mathrm{d} \mu(x) .
$$

Note that the sum is well-defined since only finitely many summands are nonzero. Consider the closed Borel set $E=\{0\} \times \mathbb{R}_{\mathrm{d}}$. On the one hand, every open set $U \subseteq$ $X$ containing $E$ has infinite measure as any open subset of $\mathbb{R}$ has positive Lebesgue measure. Hence $\mu(E)$ is infinite by outer regularity. On the other hand, $E$ is locally null as every compact set $K=\{0\} \times I \subset E$ is a null set.

However, such problems do not occur if $X$ is $\sigma$-compact or even second countable. 
Theorem 2.11. Let $X$ be a locally compact space.

(i) Suppose that $X$ is $\sigma$-compact. Then every Radon measure is regular.

(ii) Suppose that $X$ is second countable. Then every locally finite Borel measure is regular.

The first statement follows from Thm. 2.9. If $\mu$ is a Radon measure, then there exists a unique Radon measure $\mu^{\prime}$ such that

$$
\int_{X} f(x) \mathrm{d} \mu(x)=\int_{X} f(x) \mathrm{d} \mu^{\prime}(x) \quad \forall f \in C_{c}(X),
$$

which is regular. By uniqueness it follows that $\mu=\mu^{\prime}$ and hence $\mu$ is regular. The proof for the second statement can be found in Elstrodt [27, Chp. VIII, Cor. 1.12]. Another useful result is the Fubini-Tonelli Theorem.

Theorem 2.12 (Fubini-Tonelli). Let $\mu$ and $\nu$ be two $\sigma$-finite measures on locally compact spaces $X$ and $Y$.

(i) For all nonnegative measurable functions $f: X \times Y \rightarrow \mathbb{R}_{\geq 0}$, we have

$$
\int_{X}\left(\int_{Y} f(x, y) \mathrm{d} \nu(y)\right) \mathrm{d} \mu(x)=\int_{Y}\left(\int_{X} f(x, y) \mathrm{d} \mu(x)\right) \mathrm{d} \nu(y) .
$$

(ii) For all measurable functions $f: X \times Y \rightarrow \mathbb{C}$ satisfying

$$
\int_{X}\left(\int_{Y}|f(x, y)| \mathrm{d} \nu(y)\right) \mathrm{d} \mu(x)=\int_{Y}\left(\int_{X}|f(x, y)| \mathrm{d} \mu(x)\right) \mathrm{d} \nu(y)<\infty
$$

we have

$$
\int_{X}\left(\int_{Y} f(x, y) \mathrm{d} \nu(y)\right) \mathrm{d} \mu(x)=\int_{Y}\left(\int_{X} f(x, y) \mathrm{d} \mu(x)\right) \mathrm{d} \nu(y) .
$$

In the following we will frequently change the order of integration. Each time we do so, Thm. 2.12 is applied without mentioning it.

\subsubsection{Haar measure}

Let $G$ be a locally compact group. A measure $\mu$ is called left-invariant (respectively right-invariant) if $\mu(a E)=\mu(E)$ (respectively $\mu(E a)=\mu(E)$ ) for all $a \in G$ and all Borel sets $E$. One of the most important results about locally compact groups is that they admit a nontrivial left-invariant measure.

Theorem 2.13. Let $G$ be a locally compact group. Then there exists a nontrivial leftinvariant Radon measure $\mu$ on $G$, called left Haar measure. It is unique up to scalar multiples: If $\nu$ is another left Haar measure then $\mu=c \cdot \nu$ for some $c \in \mathbb{R}_{>0}$. 
Thm. 2.13 is proven by constructing a nonzero positive left-invariant functional on $C_{c}(G)$. Then Thm. 2.9 guaranties the existence of a Haar measure. Details on the construction can be found in Folland [31, Chp. 2.2].

If $\mu$ is left-invariant then $\mu_{\mathrm{r}}$, defined by $\mu_{\mathrm{r}}(E)=\mu\left(E^{-1}\right)$, is right-invariant and called a right Haar measure. In that way it is always possible to obtain a right Haar measure from a left Haar measure and vice versa. Therefore, left- and right-invariance play a symmetric role and it does not matter which one is used to describe integration on $G$. In this thesis the convention is to fix a left Haar measure $\mu$ and to write

$$
\int_{G} f(a) \mathrm{d} a:=\int_{G} f(a) \mathrm{d} \mu(a) .
$$

Integration against $\mu_{\mathrm{r}}$ is denoted by

$$
\int_{G} f(a) \mathrm{d} \mu_{\mathrm{r}}(a)=\int_{G} f(a) \mathrm{d} a^{-1} .
$$

For some $b \in G$ the translated measure $\mu_{b}$, defined by $\mu_{b}(E)=\mu(E b)$, is still leftinvariant. By uniqueness of the left Haar measure there exists a positive constant $c_{b}$ such that $\mu_{b}=c_{b} \mu$. The function $\Delta_{G}: G \rightarrow \mathbb{R}_{>0}, \Delta_{G}(b)=c_{b}$, is called the modular function of $G$. It has the following properties.

Proposition 2.14. The modular function $\Delta_{G}: G \rightarrow \mathbb{R}_{>0}$ is a continuous homomorphism. For $f \in C_{c}(G)$ and $b \in G$ it satisfies

$$
\begin{aligned}
\int_{G} f(a b) \mathrm{d} a & =\Delta_{G}\left(b^{-1}\right) \int_{G} f(a) \mathrm{d} a \\
\int_{G} f(a) \mathrm{d} a^{-1} & =\Delta_{G}\left(a^{-1}\right) \int_{G} f(a) \mathrm{d} a .
\end{aligned}
$$

If $\Delta_{G} \equiv 1$, then $G$ is called unimodular.

If $G$ is compact, then $\Delta_{G}(G)$ is a compact subgroup of $\mathbb{R}_{>0}$. As $\{1\}$ is the only compact subgroup of $\mathbb{R}_{>0}$, it follows that $G$ is unimodular.

As $\Delta_{G}$ is a homomorphism and $\mathbb{R}_{>0}$ is commutative, it follows directly that $[G, G] \unlhd G$ lies in the kernel of $\Delta_{G}$. By the same argument as before $G$ is unimodular if $G /[G, G]$ is compact. In particular, simple and semisimple groups are unimodular.

Proposition 2.15. Let $G$ be a connected Lie group. Denote the adjoint action of $G$ on its Lie algebra $\mathfrak{g}$ by $\mathrm{Ad}$, the adjoint action of $\mathfrak{g}$ on itself by ad, and the exponential map $\mathfrak{g} \rightarrow G$ by $\exp _{\mathfrak{g}}$.

Then $\Delta_{G}(a)=\operatorname{det}\left[\operatorname{Ad}_{a^{-1}}\right]$ and $\Delta_{G}\left(\exp _{\mathfrak{g}}(X)\right)=\exp \left(-\operatorname{Tr}\left[\operatorname{ad}_{X}\right]\right)$.

From Prop. 2.15 it follows that connected nilpotent Lie groups are unimodular, as by Engel's Theorem $\operatorname{ad}_{X}$ is nilpotent for all $X$ in its Lie algebra and, therefore, its trace vanishes. Nevertheless, many solvable Lie groups are nonunimodular like the Lie group of affine transformations $x \mapsto a x+b$ on $\mathbb{R}$, for example. 


\subsubsection{Function spaces}

Let $X$ be a locally compact space. Let $C(X)=C(X ; \mathbb{C})$ be the space of complexvalued continuous functions on $X . C(X)$ is endowed with the topology of locally uniform convergence. That is the topology induced by the semi-norms

$$
p_{K}(f):=\left\|\left.f\right|_{K}\right\|_{\infty}:=\sup _{x \in K}|f(x)|
$$

for all compact subsets $K \subseteq X$.

Let $C_{b}(X)=C_{b}(X ; \mathbb{C})$ be the space of continuous bounded functions on $X . C_{b}(X)$ is a linear subspace of $C(X)$ and it is endowed with topology of uniform convergence, which is induced by the norm

$$
\|f\|_{\infty}:=\sup _{x \in X}|f(x)| .
$$

$C_{c}(X)=C_{c}(X ; \mathbb{C})$ is the space of continuous compactly supported functions on $X$. For all compact subsets $K \subseteq X$ let $C_{c}(X, K)$ be the subset of $C_{c}(X)$ consisting of all functions $f \in C_{c}(X)$ with $\operatorname{supp}(f) \subseteq K$. Endowed with the topology of uniform convergence, each $C_{c}(X, K)$ is a closed subspace of $C(X)$. The space $C_{c}(X)$ is endowed with the direct limit topology

$$
C_{c}(X)=\lim _{\longrightarrow} C_{c}(X, K)
$$

over all compact subsets $K \subseteq X$.

The inclusion $C_{c}(X) \hookrightarrow C_{b}(X) \subseteq C(X)$ is continuous, however, $C_{c}(X)$ is not closed in $C(X)$ unless $X$ is compact. Its closure is denoted by $C_{0}(X)=C_{0}(X ; \mathbb{C})$ and can be described as follows. A continuous function $f$ is contained in $C_{0}(X)$ if and only if the sets $\{x \in X|| f(x) \mid>\epsilon\}$ are compact for all $\epsilon>0$. Another description comes from the one-point compactification $\bar{X}=X \cup\{\infty\}$ of $X . C_{0}(X)$ consists of all $f \in C(\bar{X})$ vanishing at $\infty$. The functions in $C_{0}(X)$ are said to vanish at infinity.

Let $G$ be a locally compact group. The left and right translation $L_{b}$ and $R_{b}$ for $b \in G$ are defined by

$$
L_{b} f(a)=f\left(b^{-1} a\right), \quad R_{b} f(a)=f(a b), \quad \forall a \in G .
$$

Both define actions of $G$ as

$$
L_{b c}=L_{b} L_{c}, \quad \quad R_{b c}=R_{b} R_{c}, \quad \forall b, c \in G .
$$

A function $f: G \rightarrow \mathbb{C}$ is called left or right uniformly continuous if

$$
\lim _{b \rightarrow e}\left\|L_{b} f-f\right\|_{\infty}=0 \quad \text { or } \quad \lim _{b \rightarrow e}\left\|R_{b} f-f\right\|_{\infty}=0,
$$


respectively. Denote the spaces of left and right uniformly continuous functions by $C_{l u}(G)$ and $C_{r u}(G)$, respectively.

Proposition 2.16. Every compactly supported continuous function is left (right) uniformly continuous. Every left (right) uniformly continuous function is continuous.

$$
C_{c}(G) \subseteq C_{l u}(G) \subseteq C(G)
$$

Proof. The proof for the first inclusion can be found in Folland [31, Prop. 2.6].

For the second part let $f \in C_{l u}(G)$. Let $a \in G$ and $\epsilon>0$. Since $f$ is left uniformly continuous, there exists an open neighborhood $V \subseteq G$ of $e_{G}$ such that $\left\|L_{b} f-f\right\|_{\infty}<\epsilon$ for all $b \in V$. Then for all $b$ in the open neighborhood $U=V^{-1} a$ of $a$ the function $f$ satisfies $|f(b)-f(a)|<\epsilon$. Therefore $f$ is continuous at any $g \in G$.

For $C_{r u}(G)$ the proof works analogously.

Prop. 2.16 can be used to show the following result.

Proposition 2.17. Let $H \leq G$ be a closed subgroup and denote the quotient space $G / H$ by $X$. For $f \in C_{c}(G)$ let $f_{H}: G \rightarrow \mathbb{C}$ be the function given by

$$
f_{H}(a)=\int_{H} f(a h) \mathrm{d} h .
$$

(i) For all compact subsets $K \subseteq G$ with nonempty interior, there exists a constant $c_{K}>0$ such that

$$
\left\|f_{H}\right\|_{\infty} \leq c_{K}\|f\|_{\infty}, \quad \forall f \in C_{c}(G, K)
$$

(ii) $f_{H}$ is continuous for all $f \in C_{c}(G)$.

(iii) There exists a unique function $f^{X} \in C_{c}(X)$ such that $f^{X}(a H)=f_{H}(a)$.

Proof.

(i) The proof of the first part can be found in Kaniuth and Taylor [44, Prop. 1.10].

(ii) Let $f \in C_{c}(G)$. By Prop. 2.16 it is sufficient to show that $f_{H}$ is left uniformly continuous. Let $\epsilon>0$. Let $K \subseteq G$ be a compact neighborhood of $e_{G}$ and denote the compact subset $K \cdot \operatorname{supp}(f) \subseteq G$ by $L$. Since $f$ is uniformly continuous, there exists an open neighborhood $U \subseteq G$ of $e_{G}$ such that

$$
\left\|L_{b} f-f\right\|_{\infty}<\frac{\epsilon}{c_{L}}
$$

for all $b \in U$. W.l.o.g. assume that $U \subseteq K$. Then for all $b \in U$ the function $L_{b} f-f$ lies in $C_{c}(G, L)$ as

$$
\operatorname{supp}\left(L_{b} f-f\right) \subseteq b \operatorname{supp}(f) \cup \operatorname{supp}(f) \subseteq L .
$$


Therefore, the function $L_{b} f_{H}-f_{H}=\left(L_{b} f-f\right)_{H}$ satisfies

$$
\left\|L_{b} f_{H}-f_{H}\right\|_{\infty} \leq c_{L}\left\|L_{b} f-f\right\|_{\infty}<\epsilon \quad \forall b \in U .
$$

(iii) Let $f \in C_{c}(G)$. By the universal property of the quotient space there exists a continuous map $f^{X}: X \rightarrow \mathbb{C}$ such that $f^{X}(a H)=f_{H}(a)$ for all $g \in G$. Since the canonical quotient map $p: G \rightarrow X$ is continuous, the subset $p(\operatorname{supp}(f))$ is compact. For $\operatorname{supp}\left(f^{X}\right) \subseteq p(\operatorname{supp}(f))$ it follows that $f^{X} \in C_{c}(X)$.

Fix some left Haar measure on $G$. For $1 \leq p<\infty$ let $L^{p}(G)=L^{p}(G$; $\mathbb{C})$ be the space of equivalence classes of measurable functions $f: G \rightarrow \mathbb{C}$ satisfying

$$
\|f\|_{p}^{p}:=\int_{G}|f(a)|^{p} \mathrm{~d} a<\infty
$$

where two measurable functions on $G$ are equivalent if they coincide on a conull subset. For all $1 \leq p<\infty, L^{p}(G)$ is a Banach space. Moreover, $L^{2}(G)$ is a Hilbert space.

$L^{\infty}(G ; \mathbb{C})=L^{\infty}(G)$ is the space of measurable functions which are essentially bounded. That is, there exists a bound $B>0$ such that

$$
\{f>B\}:=\{a \in G|| f(a) \mid>B\}
$$

is a locally null set. Again, two functions are identified if they coincide on s subset which has a locally null complement. The norm $\|f\|_{\infty}$ is defined as the infimum of all bounds $B$ for which $\{f>B\}$ is locally null.

Let $f \in L^{1}(G)$ and let $g \in L^{p}(G)$, for $1 \leq p \leq \infty$. The convolution $f * g \in L^{p}(G)$ of $f$ and $g$ is defined by

$$
(f * g)(a)=\int_{G} f(b) g\left(b^{-1} a\right) \mathrm{d} b .
$$

\subsubsection{Quasi-invariant measures on the quotient space}

In Chp. 2.1.4 we saw that every locally compact group $G$ has a left Haar measure. Of course every closed subgroup $H \leq G$ has a left Haar measure, as well, and for all $a \in G$ the positive linear functional

$$
f \mapsto \int_{H} f(a h) \mathrm{d} h
$$

on $C_{c}(a H) \rightarrow \mathbb{C}$ defines by the Riesz-Markov-Kakutani Representation Theorem (cf. Thm. 2.9p a nonzero Radon measure $\mu_{a H}$ on $a H$. By left invariance of the Haar measure on $H$, it only depends on the left coset $a H$ and not specifically on $a$.

In this chapter we study the relationship between the Haar measure of $G$ and the measures $\mu_{a H}$ on its left cosets. In particular, we investigate the existence of a measure 
$\nu$ on $G / H$ such that

$$
\int_{G} f(a) \mathrm{d} a=\int_{G / H} \int_{b H} f(a) \mathrm{d} \mu_{b H}(a) \mathrm{d} \nu(b H) \quad \forall f \in C_{c}(G) .
$$

Here, we used that $\left.f\right|_{b H} \in C_{c}(b H)$ for any $f \in C_{c}(G)$, as $b H \subseteq G$ is a closed subset. The measure $\nu$ plays an important role. If such a measure exists, then it is necessarily $G$-invariant. Conversely, if there exists a nontrivial $G$-invariant Radon measure one can show that it satisfies eq. (2.1) up to a constant positive factor.

Let $H$ be a closed subgroup of a locally compact group $G$. Let $X=G / H$ be the quotient space of left cosets and denote the canonical quotient map $G \rightarrow X$ by $p$. To begin with let $\nu$ be a Radon measure on $G / H$ and define the positive linear functional $\lambda: C_{c}(G) \rightarrow \mathbb{C}$ by

$$
\lambda(f)=\int_{X} \int_{x} f(a) \mathrm{d} \mu_{x}(a) \mathrm{d} \nu(x) .
$$

Let $\mu_{\lambda}$ be the corresponding Radon measure on $G$ and suppose that $\mu_{\lambda}$ has a continuous density with respect to the Haar measure, i.e., there is a continuous map $\rho: G \rightarrow \mathbb{R}_{\geq 0}$ such that

$$
\lambda(f)=\int_{G} f(a) \mathrm{d} \mu_{\lambda}(a)=\int_{G} f(a) \rho(a) \mathrm{d} a, \quad \forall f \in C_{c}(G) .
$$

For $f \in C_{c}(G)$ and $h \in H$, define $f_{h} \in C_{c}(G)$ by $f_{h}(a)=\Delta_{G}\left(h^{-1}\right) f\left(a h^{-1}\right)$. When computing $\lambda\left(f_{h}\right)$ we get on the one hand

$$
\lambda\left(f_{h}\right)=\int_{G} f_{h}(a) \rho(a) \mathrm{d} a=\int_{G} \Delta_{G}\left(h^{-1}\right) f\left(a h^{-1}\right) \rho(a) \mathrm{d} a=\int_{G} f(a) \rho(a h) \mathrm{d} a .
$$

On the other hand, since $f_{h}$ satisfies

$$
\begin{aligned}
\int_{b H} f_{h}(a) \mathrm{d} \mu_{b H}(a) & =\int_{H} f_{h}\left(b h^{\prime}\right) \mathrm{d} h^{\prime}=\int_{H} \Delta_{G}\left(h^{-1}\right) f\left(b h^{\prime} h^{-1}\right) \mathrm{d} h^{\prime} \\
& =\frac{\Delta_{H}(h)}{\Delta_{G}(h)} \int_{H} f\left(b h^{\prime}\right) \mathrm{d} h^{\prime}=\frac{\Delta_{H}(h)}{\Delta_{G}(h)} \int_{b H} f(a) \mathrm{d} \mu_{b H}(a),
\end{aligned}
$$

we obtain

$$
\lambda\left(f_{h}\right)=\frac{\Delta_{H}(h)}{\Delta_{G}(h)} \lambda(f)=\int_{G} f(a) \frac{\Delta_{H}(h)}{\Delta_{G}(h)} \rho(a) \mathrm{d} a .
$$

As eq. (2.3) and eq. 2.4 hold for all $f \in C_{c}(G)$, the function $\rho$ has the property that

$$
\rho(a h)=\frac{\Delta_{H}(h)}{\Delta_{G}(h)} \rho(a), \quad \forall a \in G, h \in H
$$

From eq. 2.2 and eq. 2.5 it follows that the decomposition in eq. 2.1) only exist if $\left.\Delta_{G}\right|_{H}=\Delta_{H}$. In general, there does not exist a $G$-invariant measure on $X$ and, thus, 
we have to work with a weaker class of measures, namely with quasi-invariant ones.

Definition 2.18. Let $H \leq G$ be locally compact groups and denote $G / H$ by $X$. Let $\mu$ be a Radon measure on $X$.

- Recall that for $a \in G$ the translated measure $\mu_{a}$ is given by $\mu_{a}(E)=\mu(a E)$ for all Borel sets $E \subseteq X$.

- $\mu$ is called quasi-invariant if $\mu \neq 0$ and $\mu_{a}$ is equivalent to $\mu$ for all $a \in G$.

To show that quasi-invariant measures exist we introduce the following auxiliary functions.

Definition 2.19. Let $H \leq G$ be locally compact groups. Denote $G / H$ by $X$ and the canonical quotient map $G \rightarrow X$ by $p$.

(i) A Bruhat section for $(G, H)$ is a continuous map $\beta: G \rightarrow \mathbb{R}_{\geq 0}$ with the property that $\operatorname{supp}(\beta) \cap p^{-1}(K)$ is compact for all compact subsets $K \subseteq X$ and

$$
\int_{H} \beta(a h) \mathrm{d} h=1 \quad \forall a \in G .
$$

(ii) A rho-function for $(G, H)$ is a measurable function $\rho: G \rightarrow \mathbb{R}_{\geq 0}$ which is locally integrable, i.e.,

$$
\int_{K} \rho(a) \mathrm{d} a<\infty
$$

for all compact subsets $K \subseteq G$, and with the property that

$$
\rho(a h)=\frac{\Delta_{H}(h)}{\Delta_{G}(h)} \rho(a)
$$

for locally almost all $a \in G$ and all $h \in H$.

Proposition 2.20. Let $H \leq G$ be locally compact groups. There exists a Bruhat section and a continuous strictly positive rho-function for $(G, H)$.

Proof.

(i) By Bourbaki [12, Chp. III.4.6 Prop. 13], quotient spaces of locally compact groups are paracompact and, by Bourbaki [14, Chp. IX.4.4 Cor. 1], there exists a partition of unity subordinate to any open cover. Since $G$ and $X$ are locally compact, there exist partitions of unity $\left\{\phi_{i}\right\}_{i \in I} \subseteq C_{c}(G)$ and $\left\{\psi_{j}\right\}_{j \in J} \subseteq C_{c}(X)$ of $G$ and $X$, respectively, by compactly supported functions. For every $j \in J$ there exist finitely many subsets $I_{j} \subset I$ such that

$$
\operatorname{supp}\left(\psi_{j}\right) \subseteq \bigcup_{i \in I_{j}} p\left(\left\{a \in G \mid \phi_{i}(a)>0\right\}\right)
$$


The function $\Psi_{j}: G \rightarrow[0,1]$,

$$
\Psi_{j}(a)=\psi_{j}(p(a)) \cdot \sum_{i \in I_{j}} \phi(a)
$$

is continuous and compactly supported. Moreover, it satisfies

$$
p\left(\operatorname{supp}\left(\Psi_{j}\right)\right)=\operatorname{supp}\left(\psi_{j}\right) \cap \bigcup_{i \in I_{j}} p\left(\left\{a \in G \mid \phi_{i}(a)>0\right\}\right)=\operatorname{supp}\left(\psi_{j}\right) .
$$

The function $\Psi=\sum_{j \in J} \Psi_{j}$ is well-defined and continuous, since the sum is locally finite, and $\operatorname{supp}(\Psi) \cap p^{-1}(K)$ is compact for all compact sets $K \subseteq X$. Moreover, $\tilde{\Psi}: G \rightarrow \mathbb{R}_{\geq 0}$ defined by

$$
\tilde{\Psi}(a)=\int_{H} \Psi(a h) \mathrm{d} h
$$

is well-defined, continuous and strictly positive. Therefore, the function $\beta: G \rightarrow$ $\mathbb{R}_{\geq 0}$,

$$
\beta(a)=\frac{\Psi(a)}{\tilde{\Psi}(a)}
$$

is a Bruhat section.

(ii) The function $\rho: G \rightarrow \mathbb{R}_{>0}$,

$$
\rho(a)=\int_{H} \frac{\Delta_{G}(h)}{\Delta_{H}(h)} \beta(a h) \mathrm{d} h
$$

is a strictly positive rho-function. From the property that $\operatorname{supp}(\beta) \cap p^{-1}(K)$ is compact for all compact subsets $K \subseteq X$ and by Prop. 2.17, it follows that $\rho$ is continuous.

Proposition 2.21. If $\beta$ is a Bruhat section, then $\beta_{a}: b \mapsto \beta(a b)$ is a Bruhat section, as well.

In the discussion at the beginning of this chapter we saw that there is close relation between measures on $X$ and rho-function. The following proposition makes this connection more precise.

Proposition 2.22. Let $H \leq G$ be locally compact groups and denote $G / H$ by $X$. For every rho-function $\rho$ there exists a Radon measure $\nu_{\rho}$ on $X$ such that

$$
\int_{X} \int_{x} f(b) \mathrm{d} \mu_{x}(b) \mathrm{d} \nu_{\rho}(x)=\int_{G} f(a) \rho(a) \mathrm{d} a \quad \forall f \in C_{c}(G),
$$


where $\mu_{a H}$ is the measure on $a H \subseteq G$, defined by

$$
\int_{a H} f(b) \mathrm{d} \mu_{a H}(b)=\int_{H} f(a h) \mathrm{d} h .
$$

Proof. Denote the canonical quotient map $G \rightarrow X$ by $p$ and let $\beta: G \rightarrow \mathbb{R}_{\geq 0}$ be a Bruhat section. Then, for every $\varphi \in C_{c}(X)$, the function $\beta \cdot(\varphi \circ p)$ is in $C_{c}(G)$ and is a nonnegative function whenever $\varphi$ is. Thus, the functional $\lambda_{\rho}: C_{c}(X) \rightarrow \mathbb{C}$,

$$
\lambda_{\rho}(\varphi)=\int_{G} \beta(a) \varphi(a H) \rho(a) \mathrm{d} a \quad \forall \varphi \in C_{c}(X),
$$

is well-defined and positive, and, therefore, it yields a Radon measure $\nu_{\rho}$ such that

$$
\int_{X} \varphi(x) \mathrm{d} \nu_{\rho}(x)=\lambda_{\rho}(\varphi)=\int_{G} \beta(a) \varphi(a H) \rho(a) \mathrm{d} a .
$$

It remains to show eq. 2.6). Let $f \in C_{c}(G)$ and $f^{X} \in C_{c}(X)$ as in Prop. 2.17, i.e., $f^{X}(a H)=\int_{H} f(a h) \mathrm{d} h$. Then,

$$
\begin{aligned}
\int_{X} \underbrace{\int_{x} f(a) \mathrm{d} \mu_{x}(a)}_{=f^{X}(x)} \mathrm{d} \nu_{\rho}(x) & =\int_{G} \beta(a) \underbrace{\left(\int_{H} f(a h) \mathrm{d} h\right)}_{=f^{X}(a H)} \rho(a) \mathrm{d} a \\
& =\int_{H} \int_{G} \beta(a) f(a h) \rho(a h) \Delta_{G}(h) \mathrm{d} a \Delta_{H}\left(h^{-1}\right) \mathrm{d} h \\
& =\int_{H} \int_{G} \beta\left(a h^{-1}\right) f(a) \rho(a) \mathrm{d} a \Delta_{H}\left(h^{-1}\right) \mathrm{d} h \\
& =\int_{H} \int_{G} \beta(a h) f(a) \rho(a) \mathrm{d} a \mathrm{~d} h \\
& =\int_{G} f(a) \rho(a) \mathrm{d} a .
\end{aligned}
$$

In particular, $\nu_{\rho}$ does not depend on the choice of the Bruhat section.

Theorem 2.23. Let $G$ be a locally compact group and $H \leq G$ a closed subgroup and denote $G / H$ by $X$. Let $\rho: G \rightarrow \mathbb{R}_{>0}$ be a continuous strictly positive rho-function for $(G, H)$ (guaranteed by Prop. 2.20) and let $\nu_{\rho}$ be the corresponding Radon measure corresponding to $\rho$ (cf. Prop. 2.22).

Then $\nu_{\rho}$ is quasi-invariant. In particular, there exists a continuous strictly positive function $\sigma: G \times H \rightarrow \mathbb{R}_{>0}$ such that

$$
\int_{X} f(x) \mathrm{d} \nu_{\rho}(a x)=\int_{X} f(x) \sigma(a, x) \mathrm{d} \nu_{\rho}(x) \quad \forall f \in C_{c}(X) .
$$

$\sigma$ is given by

$$
\sigma(a, b H)=\frac{\rho(a b)}{\rho(b)} \quad \forall a, b \in G
$$


and satisfies

$$
\sigma(a, b, x)=\sigma(a, b x) \sigma(b, x) \quad \forall a, b \in G, x \in X
$$

Proof. Since $\rho$ is strictly positive and continuous, the function $G \times G \rightarrow \mathbb{R}_{>0},(a, b) \mapsto$ $\frac{\rho(a b)}{\rho(b)}$ is well-defined, continuous, and strictly positive. For all $a, b \in G$ and $h \in H$ it satisfies

$$
\frac{\rho(a b h)}{\rho(b h)}=\frac{\rho(a b)}{\rho(b)}
$$

and, therefore, there exists a continuous strictly positive function $\sigma: G \times H \rightarrow \mathbb{R}_{>0}$ such that

$$
\sigma(a, b H)=\frac{\rho(a b)}{\rho(b)} \quad \forall a, b \in G
$$

By construction $\sigma$ satisfies eq. (2.8) and, therefore, eq. (2.9), which is a direct consequence of eq. 2.8).

To show eq. (2.7) let $\beta$ be a Bruhat section for $(G, H)$ and let $f \in C_{c}(X)$. Then for all $a \in G$

$$
\begin{aligned}
\int_{X} f(x) \mathrm{d} \nu_{\rho}(a x) & =\int_{X} f\left(a^{-1} x\right) \mathrm{d} \nu_{\rho}(x) \\
& =\int_{G} \beta(b) f\left(g^{-1} b H\right) \rho(b) \mathrm{d} b \\
& =\int_{G} \beta(a b) f(b H) \frac{\rho(a b)}{\rho(b)} \rho(b) \mathrm{d} b \\
& =\int_{X} f(x) \sigma(a, x) \mathrm{d} \nu_{\rho}(x),
\end{aligned}
$$

where we used that $\int_{H} \beta(a b h) \mathrm{d} h=1$ for all $a, b \in G$. In particular, it follows that $\nu_{\rho}$ is quasi-invariant.

Corollary 2.24. Let $H \leq G$ be locally compact groups and denote $G / H$ by $X$. There exists a G-invariant Radon measure on $X$ if and only if $\left.\Delta_{G}\right|_{H}=\Delta_{H}$.

\subsubsection{Standard Borel spaces}

Given a locally compact group $G$ and a closed subgroup $H \leq G$ it will turn out to be very helpful to have a measurable function $q: G / H \rightarrow G$ such that $p(q(x))=x$, where $p: G \rightarrow G / H$ denotes the quotient map. If such a function $q$ exists then every element $a \in G$ can be uniquely written as $a=q(x) h$ where $x=p(a) \in G / H$ and $h=q(p(a))^{-1} a \in H$. Moreover the map

$$
G / H \times H \rightarrow G, \quad(x, h) \mapsto q(x) h,
$$


is a measurable isomorphism and we will see that there exists a measure $\nu$ on $G / H$ such that

$$
\int_{G} f(a) \mathrm{d} a=\int_{G / H} \int_{H} f(q(x) h) \frac{\Delta_{G}(h)}{\Delta_{H}(h)} \mathrm{d} h \mathrm{~d} \nu(x) \quad \forall f \in C_{c}(G) .
$$

More generally, let $X$ and $Y$ be measurable spaces and let $p: X \rightarrow Y$ be a measurable surjective map. A measurable transversal for $p$ is a measurable subset $E \subseteq X$ which meets each of the sets $p^{-1}(y)$ for $y \in Y$ in exactly one point. A measurable crosssection is a measurable map $q: Y \rightarrow X$ satisfying $p \circ q=\mathrm{id}_{Y}$. From the algebraic point of view there is a one-to-one correspondence between transversals and cross-sections. However, if one is measurable the other one need not be measurable.

The results presented in this section are mostly due to Mackey [51]. The notions of measurable transversals and measurable cross-sections, however, are taken from Führ [33] and they are inconsistent with the notions used in [51]. Note that Mackey as well as Dixmier and other authors who published during that time use the word "separable" to refer to second countable topological spaces. Indeed, in [21, B.31] Dixmier states that a topological space is called "separable" if its topology has a countable base.

To investigate the existence of measurable transversals and cross-sections and their relation we need the following definitions, which are due to Mackey [51].

Definition 2.25. Let $X$ be a measurable space.

(i) $X$ is called countably separated if there exists a countable family of measurable subsets $\left(E_{n}\right)_{n \in \mathbb{N}}$ separating points in $X$, i.e., every point $x \in X$ satisfies

$$
\{x\}=\bigcap_{x \in E_{n}} E_{n} .
$$

(ii) $X$ is called countably generated if there exists a countable family of measurable subsets separating points in $X$ and generating the measurable structure of $X$.

(iii) $X$ is called a standard Borel space if it is measurably isomorphic to a complete separable metric space endowed with its Borel structure.

Standard Borel spaces can be considered as the Borel spaces coming from a second countable locally compact space. In fact, an even stronger characterization is true.

Theorem 2.26. Let $X$ be a standard Borel space. Then $X$ is either countable and endowed with the discrete measurable structure or $X$ is measurably isomorphic to the interval $[0,1] \subseteq \mathbb{R}$ endowed with the Borel structure generated by the standard topology of $\mathbb{R}$.

Thm. 2.26 is due to Kuratowski [47]. Conversely, there is the following result.

Theorem 2.27. Every second countable locally compact space endowed with its Borel structure is a standard Borel space. 
Proof. Let $X$ be a second countable locally compact space. By Bourbaki [14, Chp. IX.2.9, Cor. of Prop. 16] $X$ is metrizable and $\sigma$-compact. Then by Bourbaki [14, Chp. IX.6.1, Cor. of Prop. 2] $X$ is a separable and completely metrizable space.

Theorem 2.28. Let $p: X \rightarrow Y$ be a surjective measurable map from a standard Borel space $X$ to a countably separated measurable space $Y$. Then every countable separating family of measurable subsets of $Y$ generates the measurable structure of $Y$. In particular, the measurable structure of $Y$ coincides with the quotient measurable structure coming from $p: X \rightarrow Y$. I.e., a subset $E \subseteq Y$ is measurable if and only if $p^{-1}(E) \subseteq X$ is measurable.

Proof. Standard Borel spaces are countably separated as their measurable structure comes from a second countable Hausdorff topology. By [51, Thm. 3.3] every countable separating family of measurable subsets of a standard Borel space $X$ generates its measurable structure. In particular, standard Borel spaces are countably generated. From [51, Thm. 5.1] it follows that then $Y$ is countably generated and by [51, Thm. 4.3] every countable separating family of measurable subsets of $Y$ generates the measurable structure of $Y$.

If $\mathcal{E}$ is a countable separating family of measurable subsets of $Y$ then for all $E \in \mathcal{E}$ the subsets $p^{-1}(E) \subseteq X$ are measurable as $p$ is a measurable map. In particular, the sets $\mathcal{E}$ are measurable with respect to the quotient measurable structure. By the first part of the theorem $\mathcal{E}$ generates the measurable structure as well as the quotient measurable structure of $Y$. Therefore, both coincide.

Note that from Thm. 2.28 it follows in particular that standard Borel spaces are countably generated.

Theorem 2.29. Let $p: X \rightarrow Y$ be a surjective measurable map from a standard Borel space $X$ to a countably separated measurable space $Y$.

(i) If there exists a measurable transversal $E \subseteq X$, then $\left.p\right|_{E}: E \rightarrow Y$ is a measurable isomorphism and $Y$ is standard. In particular $q=\left(\left.p\right|_{E}\right)^{-1}$ is a measurable crosssection.

(ii) If there exists a measurable cross-section $q: Y \rightarrow X$ and $Y$ is standard, then $q(Y) \subseteq X$ is measurable. In particular, $q$ is a measurable isomorphism.

This theorem follows from [51, Thm. 3.2], which states the following.

Theorem 2.30. Let $p: X \rightarrow Y$ be an injective measurable map from a standard Borel space to a countably generated space. Then $p(X) \subseteq Y$ is a measurable subset and $p: X \rightarrow p(X)$ is a measurable isomorphism. In particular, $p(X)$ is a standard Borel space.

Proof of Thm.2.29. 
(i) We apply Thm. 2.30 to $\left.p\right|_{E}$. By [51, Cor. 1 of Thm. 3.2] a subset of a standard Borel space is standard if and only if it is a measurable subset. Since $X$ is standard and $E$ is measurable it follows that $E$ is a standard Borel space. As $X$ is standard and therefore countably generated, [51, Thm. 5.1] states that $Y$ is countably generated. It remains to show that $\left.p\right|_{E}$ is measurable. Let $F \subseteq Y$ be measurable. By Thm. $2.28 p^{-1}(F)$ is measurable and, therefore, $\left(\left.p\right|_{E}\right)^{-1}(F)=p^{-1}(F) \cap E$ is measurable.

(ii) We apply Thm. 2.30 to $q$. By assumption $Y$ is standard and $q$ is measurable. $X$ is countably generated as it is standard.

In [50, Lem. 1.1] Mackey shows that for second countable locally compact groups $H \leq G$ there exist measurable transversals for the quotient map $G \rightarrow G / H$.

Lemma 2.31. Let $G$ be a second countable locally compact group and $H \leq G$ a closed subgroup. Denote $G / H$ by $X$ and the canonical quotient map $G \rightarrow X$ by $p$. Then there exists a Borel set $E \subset G$ such that $E$ intersects each $H$-coset in exactly one point and for each compact subset $K \subseteq X$ the closure of $E \cap p^{-1}(K)$ is compact.

The transversal $E$ in Lem. 2.31 is called a regular transversal.

Corollary 2.32. Let $G$ be a second countable locally compact group and $H \leq G$ a closed subgroup. Denote $G / H$ by $X$ and the canonical quotient map $G \rightarrow X$ by $p$. Then there exist measurable transversals and cross-sections. Moreover, for every measurable transversal $E$ the map $\left(\left.p\right|_{E}\right)^{-1}$ is a measurable cross-section and for every measurable cross-section $q: X \rightarrow G$ the set $q(X)$ is a measurable transversal.

The measurable cross-section $q$ is called a regular cross-section if $q(X)$ is a regular transversal.

Lemma 2.33. Let $G$ be a second countable locally compact group and $H$ a closed subgroup. Denote $G / H$ by $X$ and the quotient map $G \rightarrow X$ by $p$.

(i) For every regular cross-section $q: X \rightarrow G$ there exists a quasi-invariant measure $\nu_{q}$ on $X$ such that

$$
\int_{G} f(a) \mathrm{d} a=\int_{X} \int_{H} f(q(x) h) \frac{\Delta_{G}(h)}{\Delta_{H}(h)} \mathrm{d} h \mathrm{~d} \nu_{q}(x) .
$$

(ii) For every continuous strictly positive rho-function @ there exists a regular crosssection $q: X \rightarrow G$ such that

$$
\int_{G} f(a) \mathrm{d} a=\int_{X} \int_{H} f(q(x) h) \frac{\Delta_{G}(h)}{\Delta_{H}(h)} \mathrm{d} h \mathrm{~d} \nu_{\varrho}(x) .
$$

Proof. 
(i) Given a regular Borel cross-section $q$, recall that any element $a \in G$ can be uniquely written as a product $a=q(x) h$, where $x=p(a)$ is in $X$ and $h=$ $q(p(a))^{-1} a$ is in $H$. Let $\varrho$ be a continuous strictly positive rho-function for $(G, H)$. Then for any $f \in C_{c}(G)$ we obtain

$$
\begin{aligned}
\int_{G} f(a) \mathrm{d} a & =\int_{X} \int_{x} \frac{f(a)}{\varrho(a)} \mathrm{d} \mu_{x}(a) \mathrm{d} \nu_{\varrho}(x) \\
& =\int_{X} \int_{H} \frac{f(q(x) h)}{\varrho(q(x) h)} \mathrm{d} h \mathrm{~d} \nu_{\varrho}(x) \\
& =\int_{X} \int_{H} f(q(x) h) \frac{\Delta_{G}(h)}{\Delta_{H}(h)} \mathrm{d} h \varrho(q(x))^{-1} \mathrm{~d} \nu_{\varrho}(x) .
\end{aligned}
$$

Let $\nu_{q}$ be the Radon measure corresponding to the functional

$$
f \mapsto \int_{X} f(x) \varrho(q(x))^{-1} \mathrm{~d} \nu_{\varrho}(x) \quad \forall f \in C_{c}(X) .
$$

Then

$$
\int_{G} f(a) \mathrm{d} a=\int_{X} \int_{H} f(q(x) h) \frac{\Delta_{G}(h)}{\Delta_{H}(h)} \mathrm{d} h \mathrm{~d} \nu_{q}(x) .
$$

(ii) Conversely, we can find a regular Borel cross-section $q^{\prime}: X \rightarrow G$ for $p$ such that $\varrho \circ q^{\prime} \equiv 1$ and

$$
\int_{G} f(a) \mathrm{d} a=\int_{X} \int_{H} f\left(q^{\prime}(x) h\right) \frac{\Delta_{G}(h)}{\Delta_{H}(h)} \mathrm{d} h \mathrm{~d} \nu_{\varrho}(x) .
$$

To see that, let $p_{0}=\Delta_{G} / \Delta_{H}: H \rightarrow \mathbb{R}_{>0}$ and let $H_{0}=\operatorname{ker} p_{0}$. Then there exists a regular cross-section $q_{0}: \operatorname{im} p_{0} \rightarrow H$ for $p_{0}$. Let $q^{\prime}: X \rightarrow G$ be defined by

$$
q^{\prime}(x)=q(x) q_{0}(\varrho(q(x)))
$$

If $K \subseteq X$ is compact then the closure $\overline{q_{0}(\varrho(q(K)))} \subseteq \overline{q_{0}(\varrho(\overline{q(K)}))}$ is compact as $\overline{q(K)}$ is compact and $\varrho$ is continuous. Hence, $\overline{q^{\prime}(K)}$ is compact for all compact subsets $K \subseteq X$. This implies that $q^{\prime}$ is a regular Borel cross-section for $p$. Moreover, $q^{\prime}$ satisfies

$$
\varrho\left(q^{\prime}(x)\right)=\varrho\left(q(x) q_{0}(\varrho(q(x)))\right)=\frac{\varrho(q(x))}{p_{0}\left(q_{0}(\varrho(q(x)))\right)}=\frac{\varrho(q(x))}{\varrho(q(x))}=1 .
$$

By using $q^{\prime}$ instead of $q$ we can rewrite the left Haar measure by

$$
\int_{G} f(a) \mathrm{d} a=\int_{X} \int_{H} f\left(q^{\prime}(x) h\right) \frac{\Delta_{G}(h)}{\Delta_{H}(h)} \mathrm{d} h \mathrm{~d} \nu_{\varrho}(x) .
$$




\section{$2.2 \quad$ Representation theory}

This section gives an overview of the basic notions in representation theory with particular focus on induced representations and their properties. The first part, Chp. 2.2.1. follows Folland [31, Chp. 2]. The second part follows Kaniuth and Taylor [44, Chp. 2].

\subsubsection{Unitary representation}

Let $\mathcal{H}$ be complex Hilbert space. The group of unitary operators on $\mathcal{H}$ is denoted by $\mathcal{U}(\mathcal{H})$. Let $G$ be a locally compact group. A strongly continuous unitary representation $\pi$ of $G$ on $\mathcal{H}$ is a homomorphism (of groups) from $G$ to $\mathcal{U}(\mathcal{H}$ ) which is strongly continuous, meaning that the maps $G \rightarrow \mathcal{H}$,

$$
a \mapsto \pi(a) v
$$

are continuous for all $v \in \mathcal{H}$. The scalar product of $\mathcal{H}$ is denoted by $\langle\cdot, \cdot\rangle$ and is antilinear in the first and linear in the second argument (physics convention).

A strongly continuous unitary representation is not necessarily continuous in the norm topology. However, as the strong operator topology coincides with the weak operator topology on $\mathcal{U}(\mathcal{H})$, a unitary representation is strongly continuous if and only if it is weakly continuous. That is, for all $v, w \in \mathcal{H}$ the maps $G \rightarrow \mathbb{C}$,

$$
a \mapsto\langle v, \pi(a) w\rangle
$$

are continuous. All representations which occur in this thesis will be unitary and strongly continuous. Therefore, we will often omit the adjectives and simply write representations. If not otherwise stated, the representation space of $\pi$ is denoted by $\mathcal{H}_{\pi}$. To avoid confusions we will use the notation $(\pi, \mathcal{H})$ when we want to refer to a certain Hilbert space $\mathcal{H}$.

Each locally compact group admits four important examples of representations.

Definition 2.34. Let $G$ be a locally compact group.

(i) The representation mapping every element of $G$ to $\mathbb{1}_{\mathcal{H}}$, the identity of a Hilbert space $\mathcal{H}$, is called the trivial representation.

(ii) The left regular representation $\lambda_{G}$ of $G$ on the Hilbert space $L^{2}(G)$ is given by

$$
\left[\lambda_{G}(b) f\right](a)=f\left(b^{-1} a\right) \quad \forall f \in L^{2}(G) .
$$

(iii) The right regular representation $\rho_{G}$ of $G$ on the Hilbert space $L^{2}(G)$ is given 
by

$$
\left[\rho_{G}(b) f\right](a)=\Delta(b)^{\frac{1}{2}} f(a b) \quad \forall f \in L^{2}(G) .
$$

(iv) The two-sided regular representation $\tau_{G}$ of $G \times G$ on the Hilbert space $L^{2}(G)$ is given by

$$
\left[\tau_{G}(b, c) f\right](a)=\Delta(c)^{\frac{1}{2}} f\left(b^{-1} a c\right) \quad \forall f \in L^{2}(G) .
$$

Among the linear mappings between Hilbert spaces, those which are compatible with the group action play a special role in representation theory.

Definition 2.35. Let $G$ be a locally compact group and let $\pi, \pi_{1}, \pi_{2}$ be unitary representations of $G$ on Hilbert spaces $\mathcal{H}, \mathcal{H}_{1}, \mathcal{H}_{2}$, respectively.

(i) A bounded operator $T \in \mathcal{B}\left(\mathcal{H}_{1}, \mathcal{H}_{2}\right)$ is called an intertwiner for $\pi_{1}$ and $\pi_{2}$ if it satisfies

$$
T \pi_{1}(a)=\pi_{2}(a) T \quad \forall a \in G
$$

(ii) The space of all intertwiners is denoted by $\mathcal{C}\left(\pi_{1}, \pi_{2}\right)$. It is a closed linear subspace of $\mathcal{B}\left(\mathcal{H}_{1}, \mathcal{H}_{2}\right)$.

(iii) If there exists an isometry $T \in \mathcal{C}\left(\pi_{1}, \pi_{2}\right)$ then $\pi_{1}$ is called a subrepresentation of $\pi_{2}$ and we write $\pi_{1} \leq \pi_{2}$.

(iv) If there exists a unitary operator $U \in \mathcal{C}\left(\pi_{1}, \pi_{2}\right)$ then $\pi_{1}$ and $\pi_{2}$ are called unitarily equivalent or simply equivalent, and we write $\pi_{1} \cong \pi_{2}$.

(v) The space $\mathcal{C}(\pi):=\mathcal{C}(\pi, \pi)$ is called the commutant of $\pi$. It consists of all operators $T \in \mathcal{B}(\mathcal{H}):=\mathcal{B}(\mathcal{H}, \mathcal{H})$ which commute with the operators $\pi(a)$ for all $a \in G$.

One of the main goals of representation theory is to analyze the structure of representations. In particular, the focus is on the decomposition into atoms, i.e., into irreducible ones.

Definition 2.36. Let $G$ be a locally compact group and $\pi$ a unitary representation of $G$ on a Hilbert space $\mathcal{H}$. Let $\mathcal{H}_{0}$ be closed subspace of $\mathcal{H}$.

(i) $\mathcal{H}_{0}$ is called invariant under $\pi$ if $\pi(a) \mathcal{H}_{0} \subseteq \mathcal{H}_{0}$ for all $a \in G$.

(ii) If $\mathcal{H}_{0} \neq\{0\}$ and $\mathcal{H}_{0}$ is an invariant subspace of $\mathcal{H}$ then the restriction of $\pi$ to $\mathcal{H}_{0}$ is a subrepresentation of $\pi$ and denoted by $\left.\pi\right|_{\mathcal{H}_{0}}$.

(iii) $\pi$ is called irreducible if the only invariant subspaces of $\mathcal{H}$ are $\{0\}$ and $\mathcal{H}$. Otherwise, $\pi$ is called reducible. 
The following theorem is one of the most important results for checking if a given representation is irreducible or not.

Theorem 2.37 (Schur's Lemma).

Let $G$ be a locally compact group.

(i) A unitary representation $\pi$ of $G$ is irreducible if and only if $\mathcal{C}(\pi)=\mathbb{C} \cdot \mathbb{1}$.

(ii) If $\pi_{1}$ and $\pi_{2}$ are irreducible unitary representations of $G$, then $\pi_{1}$ and $\pi_{2}$ are equivalent if and only if $\mathcal{C}\left(\pi_{1}, \pi_{2}\right)$ is one-dimensional. If $\pi_{1}$ and $\pi_{2}$ are not equivalent then $\mathcal{C}\left(\pi_{1}, \pi_{2}\right)=\{0\}$.

Definition 2.38. Let $G$ be a locally compact group and $\pi$ a unitary representation of $G$ on a Hilbert space $\mathcal{H}$. Denote the dual space of $\mathcal{H}$ by $\overline{\mathcal{H}}$. The contragredient representation $\bar{\pi}$ of $\pi$ on $\overline{\mathcal{H}}$ is given by

$$
\bar{\pi}(a) \phi:=\phi \pi\left(a^{-1}\right)=\phi \pi(a)^{*}
$$

for all $a \in G$ and $\phi \in \overline{\mathcal{H}}$.

$\pi$ and $\pi$ are not necessarily equivalent. By Schur's Lemma, $\pi$ is irreducible if and only if $\bar{\pi}$ is.

Conversely, instead of passing to subrepresentations, one can also construct bigger representations using direct sums or tensor products. If $\left(\mathcal{H}_{1},\|\cdot\|_{1}\right)$ and $\left(\mathcal{H}_{2},\|\cdot\|_{2}\right)$ are two Hilbert spaces, then $\mathcal{H}_{1} \oplus \mathcal{H}_{2}$ with the norm

$$
\left\|v_{1}+v_{2}\right\|_{\oplus}^{2}:=\left\|v_{1}\right\|_{1}^{2}+\left\|v_{2}\right\|_{2}^{2}, \quad \forall v_{1} \in \mathcal{H}_{1}, v_{2} \in \mathcal{H}_{2},
$$

is a Hilbert space. The tensor product $\mathcal{H}_{1} \otimes \mathcal{H}_{2}$ is the space of all bounded operators $T \in \mathcal{B}\left(\overline{\mathcal{H}_{2}}, \mathcal{H}_{1}\right)$ with finite norm

$$
\|T\|_{\otimes}^{2}:=\operatorname{Tr}\left[T^{*} T\right]=\operatorname{Tr}\left[T T^{*}\right]<\infty .
$$

Within this picture a decomposable tensor $v_{1} \otimes v_{2}$, with $v_{1} \in \mathcal{H}_{1}, v_{2} \in \mathcal{H}_{2}$ corresponds to the map

$$
\left(v_{1} \otimes v_{2}\right)(f)=v_{1} \cdot f\left(v_{2}\right), \quad \forall f \in \overline{\mathcal{H}_{2}} .
$$

Remark 2.39. Note that this definition of tensor products of Hilbert spaces differs from the one given in Folland [31, Chp. A.3]. Therein the tensor product of Hilbert spaces $\mathcal{H}_{1}, \mathcal{H}_{2}$ is the set of all antilinear bounded operators $T: \mathcal{H}_{2} \rightarrow \mathcal{H}_{1}$ satisfying eq. $(2.10)$. Since there exists an antilinear isomorphism between $\overline{\mathcal{H}_{2}}$ and $\mathcal{H}_{2}$, both definitions are isomorphic.

Definition 2.40. Let $G$ be a locally compact group and let $\pi_{1}, \pi_{2}$ be unitary representations of $G$ on Hilbert spaces $\mathcal{H}_{1}, \mathcal{H}_{2}$, respectively. 
(i) The direct sum $\pi=\pi_{1} \oplus \pi_{2}$ of $\pi_{1}$ and $\pi_{2}$ is the unitary representation of $G$ on $\mathcal{H}_{1} \oplus \mathcal{H}_{2}$ given by

$$
\pi(a)\left(v_{1}+v_{2}\right)=\pi_{1}(a) v_{1}+\pi_{2}(a) v_{2}
$$

for all $a \in G$ and $v_{1} \in \mathcal{H}_{1}, v_{2} \in \mathcal{H}_{2}$.

(ii) The (inner) tensor product $\pi=\pi_{1} \otimes \pi_{2}$ of $\pi_{1}$ and $\pi_{2}$ is the unitary representation of $G$ on $\mathcal{H}_{1} \otimes \mathcal{H}_{2}$ given by

$$
\pi(a)\left(v_{1} \otimes v_{2}\right)=\pi_{1}(a) v_{1} \otimes \pi_{2}(a) v_{2}
$$

for all $a \in G$ and $v_{1} \in \mathcal{H}_{1}, v_{2} \in \mathcal{H}_{2}$.

(iii) If $\left(\pi_{1}, \mathcal{H}_{1}\right)$ and $\left(\pi_{2}, \mathcal{H}_{2}\right)$ are representations of two locally compact groups $G_{1}$ and $G_{2}$, then the (outer) tensor product $\pi=\pi_{1} \times \pi_{2}$ of $\pi_{1}$ and $\pi_{2}$ is the unitary representation of $G_{1} \times G_{2}$ on $\mathcal{H}_{1} \otimes \mathcal{H}_{2}$ defined by

$$
\pi\left(a_{1}, a_{2}\right)\left(v_{1} \otimes v_{2}\right)=\pi_{1}\left(a_{1}\right) v_{1} \otimes \pi_{2}\left(a_{2}\right) v_{2}
$$

for all $a_{1} \in G_{1}, a_{2} \in G_{2}$ and $v_{1} \in \mathcal{H}_{1}, v_{2} \in \mathcal{H}_{2}$.

Hilbert spaces have the property that every closed subspace has an orthogonal complement. As a consequence, any proper subrepresentation of a unitary representation has a complement, as well.

Proposition 2.41. Let $G$ be a locally compact group and $\pi$ a unitary representation of $G$ on a Hilbert space $\mathcal{H}$. Let $\mathcal{H}_{0}$ be a nontrivial invariant subspace of $\mathcal{H}$ (meaning $\left.\{0\} \subsetneq \mathcal{H}_{0} \subsetneq \mathcal{H}\right)$. Then the orthogonal complement $\mathcal{H}_{0}^{\perp}$ is a nontrivial invariant subspace of $\mathcal{H}$ and

$$
\left.\left.\pi \cong \pi\right|_{\mathcal{H}_{0}} \oplus \pi\right|_{\mathcal{H}_{0}^{\perp}}
$$

\subsubsection{Induced representations}

The aim of the inducing procedure is to construct a unitary representation $\left(\pi, \mathcal{H}_{\pi}\right)$ of $G$ from a given unitary representation $\left(\chi, \mathcal{H}_{\chi}\right)$ of a closed subgroup $H \leq G$. The idea goes back to Frobenius [32] who developed the concept to obtain irreducible unitary representations of finite groups from subgroups which are well studied. During the last century the idea was developed much further and became one of the most important techniques in representation theory and abstract harmonic analysis. In this section we follow Kaniuth and Taylor [44.

There are different ways to describe induced representations. In the following let $G$ be a locally compact group and $H$ be a closed subgroup of $G$. Denote the quotient space 
$G / H$ by $X$ and its quotient map $G \rightarrow X$ by $p$. Let $\delta=\delta_{H}^{G}=\frac{\left.\Delta_{G}\right|_{H}}{\Delta_{H}}: H \rightarrow \mathbb{R}_{>0}$ and let $\chi$ be a unitary representation of $H$.

Variant 1. The first variant of induced representations, we will use, is the abstract version. The representation space is constructed as follows.

Let $\mathcal{F}\left(G, H, \delta^{\frac{1}{2}} \chi\right)$ be the set of all functions $F: G \rightarrow \mathcal{H}_{\chi}$ satisfying

- $F$ is continuous (with respect to the norm topology on $\mathcal{H}_{\chi}$ ),

- $p(\operatorname{supp}(F))$ is compact in $X$,

- $F(a h)=\delta\left(h^{-1}\right)^{\frac{1}{2}} \chi\left(h^{-1}\right) F(a)$, for all $a \in G$ and $h \in H$.

For all $F_{1}, F_{2} \in \mathcal{F}\left(G, H, \delta^{\frac{1}{2}} \chi\right)$ the function

$$
a \mapsto\left\langle F_{1}(a), F_{2}(a)\right\rangle_{\chi}
$$

is a rho-function and, therefore, there exists a Radon measure $\nu_{F_{1}, F_{2}}$ on $X$ satisfying

$$
\int_{X} \varphi(x) \mathrm{d} \nu_{F_{1}, F_{2}}(x)=\int_{G} \beta(a) \varphi(p(a))\left\langle F_{1}(a), F_{2}(a)\right\rangle_{\chi} \mathrm{d} a
$$

for all $\varphi \in C_{c}(X)$ and some Bruhat section $\beta: G \rightarrow \mathbb{R}_{\geq 0}$. The measure $\nu_{F_{1}, F_{2}}(x)$ is finite as

$$
\nu_{F_{1}, F_{2}}(X)=\int_{G} \beta(a)\left\langle F_{1}(a), F_{2}(a)\right\rangle_{\chi} \mathrm{d} a
$$

and $a \mapsto \beta(a)\left\langle F_{1}(a), F_{2}(a)\right\rangle_{\chi}$ is a compactly supported continuous function. It is straightforward to check that the bilinear form

$$
\left\langle F_{1}, F_{2}\right\rangle=\nu_{F_{1}, F_{2}}(X)=\int_{G} \beta(a)\left\langle F_{1}(a), F_{2}(a)\right\rangle_{\chi} \mathrm{d} a
$$

defines an inner product on $\mathcal{F}\left(G, H, \delta^{\frac{1}{2}} \chi\right)$, which is independent of the choice of the Bruhat section. By completing $\mathcal{F}\left(G, H, \delta^{\frac{1}{2}} \chi\right)$ we obtain a Hilbert space, which we denote by $L^{2}\left(G, H, \delta^{\frac{1}{2}} \chi\right)$. The space $L^{2}\left(G, H, \delta^{\frac{1}{2}} \chi\right)$ consists of all functions $F: G \rightarrow \mathcal{H}_{\chi}$ satisfying

- $F$ is measurable and $\rho_{F}: a \mapsto\|F(a)\|_{\chi}^{2}$ is a rho-function,

- and the measure $\nu_{\rho_{F}}$ on $X$ corresponding to the rho-function $\rho_{F}$ is finite.

The norm of $F$ is given by

$$
\|F\|^{2}=\nu_{\rho_{F}}(X)=\int_{G} \beta(a)\|F(a)\|_{\chi}^{2} \mathrm{~d} a
$$

for any Bruhat section $\beta$. 
$G$ acts on $L^{2}\left(G, H, \delta^{\frac{1}{2}} \chi\right)$ by

$$
\left[\pi_{1}(a) F\right](b)=F\left(a^{-1} b\right)
$$

for all $a \in G$ and almost all $b \in G$. $\pi_{1}$ is a unitary as

$$
\left\|\pi_{1}(a) F\right\|^{2}=\int_{G} \beta(b)\left\|F\left(a^{-1} b\right)\right\|_{\chi}^{2} \mathrm{~d} b=\int_{G} \beta(a b)\|F(b)\|_{\chi}^{2} \mathrm{~d} b=\|F\|^{2} .
$$

Therefore, $\pi_{1}$ is a unitary representation.

Variant 2. The second variant is more natural because it makes directly use of the measure decomposition of the Haar measure of $G$ over the Haar measure of $H$ and a quasi-invariant measure on $X$ (see Prop. 2.22). However, an additional ingredient is necessary, namely a fixed rho-function. Moreover, it is not obvious that the resulting representation does not depend on the choice of this rho-function.

Let $\rho$ be a continuous strictly positive rho-function for $(G, H)$ and let $\mu_{\rho}$ be the corresponding quasi-invariant measure on $X$. Let $\mathcal{F}_{\rho}(G, H, \chi)$ be the set of all functions $F: G \rightarrow \mathcal{H}_{\chi}$ satisfying

- $F$ is continuous (with respect to the norm topology on $\mathcal{H}_{\chi}$ ),

- $p(\operatorname{supp}(F))$ is compact in $X$,

- $F(x h)=\chi\left(h^{-1}\right) F(x)$, for all $x \in G$ and $h \in H$.

For all $F_{1}, F_{2} \in \mathcal{F}_{\rho}(G, H, \chi)$ the function

$$
a \mapsto\left\langle F_{1}(a), F_{2}(a)\right\rangle_{\chi}
$$

is constant on cosets $a H \subseteq G$ and, therefore, there exists a compactly supported continuous function $\varphi_{F_{1}, F_{2}} \in C_{c}(X)$ such that

$$
\varphi_{F_{1}, F_{2}}(p(a))=\left\langle F_{1}(a), F_{2}(a)\right\rangle_{\chi} \quad \forall a \in G .
$$

The inner product on $\mathcal{F}_{\rho}(G, H, \chi)$ is defined by

$$
\left\langle F_{1}, F_{2}\right\rangle_{\rho}=\int_{X} \varphi_{F_{1}, F_{2}}(x) \mathrm{d} \nu_{\rho}(x)
$$

and depends on the choice of the rho-function. Let $L_{\rho}^{2}(G, H, \chi)$ be the Hilbert space obtained by completing $\mathcal{F}_{\rho}(G, H, \chi)$. G acts on $L_{\rho}^{2}(G, H, \chi)$ by

$$
\left[\pi_{\rho}(a) F(b)\right]=\left(\frac{\rho\left(a^{-1} b\right)}{\rho(b)}\right)^{\frac{1}{2}} F\left(a^{-1} b\right)
$$


which is unitary as

$$
\left\|\pi_{\rho}(a) F\right\|_{\rho}^{2}=\int_{X} \sigma\left(a^{-1}, x\right) \varphi_{F, F}\left(a^{-1} x\right) \mathrm{d} \nu_{\rho}(x)=\int_{X} \varphi_{F, F}(x) \mathrm{d} \nu_{\rho}(x)=\|F\|_{\rho}^{2},
$$

where we used that

$$
\mathrm{d} \nu_{\rho}\left(a^{-1} b H\right)=\sigma\left(a^{-1}, b H\right) \mathrm{d} \nu_{\rho}(b H)=\frac{\rho\left(a^{-1} b\right)}{\rho(b)} \mathrm{d} \nu_{\rho}(b H)
$$

(cf. Thm. 2.23).

Although, the Hilbert spaces $L_{\rho}^{2}(G, H, \chi)$ and the representations $\pi_{\rho}$ depend on the choice of the rho-function $\rho$, the representations $\left(\pi_{\rho}, L_{\rho}^{2}(G, H, \chi)\right)$ are unitarily equivalent and, in particular, equivalent to $\pi_{1}$ constructed in Variant 1. The unitary equivalence $L^{2}\left(G, H, \delta^{\frac{1}{2}} \chi\right) \rightarrow L_{\rho}^{2}(G, H, \chi)$ is induced by the map $\mathcal{F}\left(G, H, \delta^{\frac{1}{2}} \chi\right) \rightarrow \mathcal{F}_{\rho}(G, H, \chi)$,

$$
F \mapsto \rho^{\frac{1}{2}} F
$$

for all $F \in \mathcal{F}\left(G, H, \delta^{\frac{1}{2}} \chi\right)$.

Variant 3. Suppose that $G$ is second countable. By Cor. 2.32 there exists a regular Borel cross-section $q: X \rightarrow G$ of $p$ and by Lem. 2.33 there exists a quasi-invariant measure $\nu_{q}$ such that

$$
\int_{G} f(a) \mathrm{d} a=\int_{X} \int_{H} f(q(x) h) \delta(h) \mathrm{d} h \mathrm{~d} \nu_{q}(x)
$$

for all $f \in C_{c}(G)$.

Let $L_{q}^{2}(X, \chi)$ be the space of measurable functions $f: X \rightarrow \mathcal{H}_{\chi}$ such that

$$
\|f\|_{q}^{2}:=\int_{X}\|f(x)\|_{\chi}^{2} \mathrm{~d} \nu_{q}(x)<\infty
$$

$G$ acts on $L_{q}^{2}(X, \chi)$ by

$$
\left[\pi_{q}(a) f\right](x)=\delta\left(q(x)^{-1} a q\left(a^{-1} x\right)\right)^{\frac{1}{2}} \chi\left(q(x)^{-1} a q\left(a^{-1} x\right)\right) f\left(a^{-1} x\right)
$$

which is unitary as

$$
\begin{aligned}
\left\|\pi_{q}(a) f\right\|_{q}^{2} & =\int_{X} \delta\left(q(x)^{-1} a q\left(a^{-1} x\right)\right)\left\|f\left(a^{-1} x\right)\right\|_{\chi}^{2} \mathrm{~d} \nu_{q}(x) \\
& =\int_{X} \frac{\rho_{q}\left(a^{-1} q(x)\right)}{\rho_{q}(q(x))}\left\|f\left(a^{-1} x\right)\right\|_{\chi}^{2} \mathrm{~d} \nu_{q}(x) \\
& =\int_{X}\|f(x)\|_{\chi}^{2} \mathrm{~d} \nu_{q}(x)=\|f\|_{q}^{2},
\end{aligned}
$$


where we used that $\rho_{q}(q(x))=1$ for all $x \in X$ and

$$
\begin{aligned}
\delta\left(q(x)^{-1} a q\left(a^{-1} x\right)\right) & =\delta\left(q(x)^{-1} a q\left(a^{-1} x\right)\right) \underbrace{\frac{\rho_{q}\left(q\left(a^{-1} x\right)\right)}{\rho_{q}(q(x))}}_{=1} \\
& =\frac{\Delta_{H}\left(q\left(a^{-1} x\right)^{-1} a^{-1} q(x)\right)}{\Delta_{G}\left(q\left(a^{-1} x\right)^{-1} a^{-1} q(x)\right)} \frac{\rho_{q}\left(q\left(a^{-1} x\right)\right)}{\rho_{q}(q(x))}=\frac{\rho_{q}\left(a^{-1} q(x)\right)}{\rho_{q}(q(x))} .
\end{aligned}
$$

The representations $\pi_{q}$ depend on the choice of $q$ or, to be more precisely, on $\rho_{q}$. Again, they are unitarily equivalent and equivalent to $\pi_{1}$ defined in Variant 1. The unitary equivalence is given by, $L^{2}\left(G, H, \delta^{\frac{1}{2}} \chi\right) \rightarrow L_{q}^{2}(X, \chi)$,

$$
f \mapsto f \circ q .
$$

Since all of these representations are equivalent we define the induced representation as follows.

Definition 2.42. Let $H \leq G$ be a closed subgroup of a locally compact group $G$ and let $\chi$ be a unitary representation of $H$.

The representation $\pi_{1}$ constructed in Variant 1 is denoted by

$$
\operatorname{ind}_{H}^{G}(\chi)
$$

In application, it will depend on the situation which variant of the induced representation will be used. Variant $\mathbf{1}$ is very useful for technical applications and proofs dealing with groups which are not necessarily second countable. Variant $\mathbf{2}$ and Variant $\mathbf{3}$ are quite handy for explicit computations, in particular when dealing with second countable groups. However, they depend on the choice of a quasi-invariant measure on $X$ or on the choice of a Borel section $X \rightarrow G$.

\subsubsection{Properties of induced representations}

The reason why the inducing procedure is so powerful is that in many cases it behaves as on would expect. Among others, it has the following properties, which will turn out to be very helpful. Proofs and details can be found in Kaniuth and Taylor [44].

The first property is that the contragredient of an induced representation is the representation induced from the contragredient one.

Lemma 2.43. Let $\chi$ be a representation of a closed subgroup $H$ of $G$. Then

$$
\operatorname{ind}_{H}^{G}(\bar{\chi}) \cong \overline{\operatorname{ind}_{H}^{G}(\chi)} .
$$

The second property is that representations induced from direct sums are direct sums of representations induced from the individual ones. 
Lemma 2.44. Let $H$ be a closed subgroup of the locally compact group $G$, and let $\chi_{i}$, $i \in I$, be any family of unitary representations of $H$. Then

$$
\operatorname{ind}_{H}^{G}\left(\bigoplus_{i \in I} \chi_{i}\right) \cong \bigoplus_{i \in I} \operatorname{ind}_{H}^{G}\left(\chi_{i}\right)
$$

In particular, if $\operatorname{ind}_{H}^{G}(\chi)$ is an irreducible representation of $G$, then $\chi$ is an irreducible one of $H$. The converse is not true.

The next property is called induction in stages. It states the following.

Theorem 2.45. Let $H$ be a closed subgroup of $G$ and let $H_{0}$ be a closed subgroup of $H$. Let $\chi_{0}$ be a representation of $H_{0}$. Then

$$
\operatorname{ind}_{H_{0}}^{G}\left(\chi_{0}\right) \cong \operatorname{ind}_{H}^{G}\left(\operatorname{ind}_{H_{0}}^{H}\left(\chi_{0}\right)\right)
$$

The last property states that the inducing procedure is compatible with taking outer tensor products of representations.

Theorem 2.46. Let $G_{1}$ and $G_{2}$ be locally compact groups and let $H_{1} \leq G_{1}$ and $H_{2} \leq$ $G_{2}$ be closed subgroups. Let $\chi_{1}$ and $\chi_{2}$ be representations of $H_{1}$ and $H_{2}$, respectively. Then

$$
\operatorname{ind}_{H_{1} \times H_{2}}^{G_{1} \times G_{2}}\left(\chi_{1} \times \chi_{2}\right) \cong \operatorname{ind}_{H_{1}}^{G_{1}}\left(\chi_{1}\right) \times \operatorname{ind}_{H_{2}}^{G_{2}}\left(\chi_{2}\right)
$$

\subsection{The dual and the quasi-dual of a locally compact group}

The aim of this section is to describe the decomposition of representations into (multiples of) irreducible ones. Unfortunately, such a decomposition is not always unique and in those case it is not expedient to do that. However, we will see that it is always possible to get a decomposition into so-called primary representations, which are close to being (multiples of) irreducible ones. Indeed, this decomposition is unique and will turn out to be more useful.

The results presented here are taken from Folland [31, Chp. 7] and Dixmier [21, Chp. 5, Chp. 8]. The notation is mostly taken from Führ [33]. The main result of this section is the Plancherel Theorem. The version for unimodular groups and its proof can be found in Dixmier [21, Chp. 18]. The Plancherel Theorem for nonunimodular groups is taken from Tatsuuma [58] and Duflo and Moore [25].

\subsubsection{Von Neumann algebras}

There are several types of algebras which play an important role in representation theory. One of them are von Neumann algebras. 
Definition 2.47. $A{ }^{*}$-algebra is a $\mathbb{C}$-algebra endowed with a involution. That is a map $a \mapsto a^{*}$ satisfying

$$
(a+b)^{*}=a^{*}+b^{*}, \quad(\lambda a)^{*}=\bar{\lambda} a^{*}, \quad(a b)^{*}=b^{*} a^{*}, \quad\left(a^{*}\right)^{*}=a,
$$

for all $a, b \in A, \lambda \in \mathbb{C}$.

$A$ von Neumann algebra is a ${ }^{*}$-subalgebra of the bounded linear operators $\mathcal{B}(\mathcal{H})$ on some Hilbert space $\mathcal{H}$ which contains $\mathbb{1}_{\mathcal{H}}$ and is closed in the weak operator topology.

Von Neumann algebras often appear as commutants or bicommutants. Let $\mathcal{H}$ be a Hilbert space and let $S \subseteq \mathcal{B}(\mathcal{H})$ be a subset. The commutant of $S$ is the set

$$
S^{\prime}=\{T \in \mathcal{B}(\mathcal{H}) \mid S T=T S\}
$$

Correspondingly, the bicommutant $S^{\prime \prime}$ of $S$ is the commutant of the commutant of $S$, i.e., $S^{\prime \prime}=\left(S^{\prime}\right)^{\prime}$. If $S$ is closed under conjugation, i.e., $S^{*}=S$, then it is an easy exercise to prove that $S^{\prime}$ and $S^{\prime \prime}$ are von Neumann algebras.

Commutants or bicommutants not only give easy examples of von Neumann algebras. Via the famous von Neumann Density Theorem they provide a tool to verify that a given algebra is a von Neumann algebra.

Theorem 2.48 (Von Neumann Density Theorem). Let $\mathcal{H}$ be a Hilbert space and let $A$ be ${ }^{*}$-subalgebra of $\mathcal{B}(\mathcal{H})$ containing $\mathbb{1}_{\mathcal{H}}$.

Then, the following are equivalent.

- $A$ is a von Neumann algebra.

- A is closed in the strong operator topology.

- A satisfies $A=A^{\prime \prime}$.

The center of a von Neumann algebra $A$ is the commutative von Neumann algebra

$$
\mathcal{Z}(A)=A \cap A^{\prime}=A^{\prime} \cap A^{\prime \prime}
$$

Commutative von Neumann algebras admit a spectral decomposition (cf. Dixmier [22, I.7.3 Thm. 1]).

Theorem 2.49. Let $\mathcal{H}$ be a separable Hilbert space and let $A \subseteq \mathcal{B}(\mathcal{H})$ be a commutative von Neumann algebra. Then there exists a standard Borel space $M$ and a projectionvalued measure $P$ such that the map

$$
f \mapsto \int_{M} f(m) \mathrm{d} P(m)
$$

defines an isometric isomorphism from $L^{\infty}(M, \mu)$ to $A$. 
The connection between von Neumann algebras and representation theory can be illustrated as follows.

Proposition 2.50. Let $(\pi, \mathcal{H})$ be a representation of a second countable locally compact group $G$ on a separable Hilbert space $\mathcal{H}$. Then $\mathcal{Z}(\pi)=\mathcal{C}(\pi) \cap \mathcal{C}(\pi)^{\prime}=\pi(G)^{\prime} \cap \pi(G)^{\prime \prime}$ is a commutative von Neumann algebra.

Let $P$ be the projection-valued measure on the standard Borel space $M$ associated to $\mathcal{Z}(\pi)$ as in Thm. 2.49, For any measurable subset $E \subseteq M, P_{E}=P(E)$ is an orthogonal projection onto a closed subspace of $\mathcal{H}_{E}=P_{E}(\mathcal{H}) \subseteq \mathcal{H}$. In particular, if $\mathcal{H}_{E}$ is nontrivial, then $\left(\pi_{E}, \mathcal{H}_{E}\right)$ defined by $\pi_{E}(a)=P \pi(a) P^{*}$ is a subrepresentation of $\pi$.

From Schur's Lemma it follows that, for every irreducible representation $(\sigma, \mathcal{H})$ of a second countable locally compact group $G$, the algebra $\mathcal{Z}(\sigma)$ has the form

$$
\mathcal{Z}(\sigma)=\mathbb{C} \cdot \mathbb{1}_{\mathcal{H}}
$$

Therefore, $M$ only consists of one point. In fact, this is what we would expect from Prop. 2.50 as $\sigma$ has no subrepresentations. The converse is not true. For $d \in \overline{\mathbb{N}}=$ $\mathbb{N} \cup\{\infty\}$ let $\mathcal{H}_{d}$ be the separable Hilbert space of dimension $d$. To be more precise, we say that

$$
\begin{aligned}
\mathcal{H}_{d} & =\mathbb{C}^{d} \\
\mathcal{H}_{\infty} & =l^{2}(\mathbb{N})=\left\{\left.\left(a_{n}\right)_{n \in \mathbb{N}} \in \mathbb{C}^{\mathbb{N}}\left|\sum_{n \in \mathbb{N}}\right| a_{n}\right|^{2}<\infty\right\}
\end{aligned} \quad \text { for } d \in \mathbb{N}
$$

For any representation $(\pi, \mathcal{H})$ let

$$
d \cdot \mathcal{H}=\mathcal{H} \otimes \mathcal{H}_{d}, \quad d \cdot \pi=\pi \otimes \mathbb{1}_{d}
$$

be the multiple of $\mathcal{H}$ and $\pi$.

Proposition 2.51. Let $\pi$ be a representation of $G$ and $d \in \overline{\mathbb{N}}$. Then $\mathcal{Z}(\pi) \cong \mathcal{Z}(d \cdot \pi)$.

This is because

$$
\begin{aligned}
\mathcal{C}(d \cdot \pi) & =\{S \otimes T \mid S \in \mathcal{C}(\pi), T \in \mathcal{B}(\mathcal{H})\}^{\prime \prime} \\
\mathcal{C}(d \cdot \pi)^{\prime} & =\left\{S \otimes \mathbb{1}_{d} \mid S \in \mathcal{C}(\pi)^{\prime}\right\}
\end{aligned}
$$

From Prop. 2.51 it follows that, if $\sigma$ is irreducible, then $\mathcal{Z}(d \cdot \sigma)=\mathbb{C} \cdot \mathbb{1}_{d \cdot \mathcal{H}}$ even though $d \cdot \sigma$ is not irreducible. So in some sense $\mathcal{Z}$ has information about subrepresentations of a representation $\pi$ but it does not count multiplicity.

Definition 2.52. Let $G$ be a locally compact group. A representation $(\pi, \mathcal{H})$ of $G$ is called a factor representation (or primary representation) if

$$
\pi(G)^{\prime} \cap \pi(G)^{\prime \prime}=\mathbb{C} \cdot \mathbb{1}_{\mathcal{H}} .
$$


Factor representations are those for which the measure space $M$ consists only of one point.

\section{Definition 2.53.}

- Two representations $\pi_{1}$ and $\pi_{2}$ are disjoint if they have no nontrivial common subrepresentation.

- Two representations $\pi_{1}$ and $\pi_{2}$ are quasi-equivalent if there exists no subrepresentation $\rho_{1} \leq \pi_{1}$ such that $\mathcal{C}\left(\rho_{1}, \pi_{2}\right)=0$, and no subrepresentation $\rho_{2} \leq \pi_{2}$ such that $\mathcal{C}\left(\rho_{2}, \pi_{1}\right)=0$.

In that case we write $\pi_{1} \approx \pi_{2}$.

Proposition 2.54. Two factor representations are either quasi-equivalent or disjoint.

One might expect or at least hope that a representation is a factor representation if and only if it is a multiple of an irreducible representation. Unfortunately, this is not always the case.

Definition 2.55. A factor representation which is quasi-equivalent to an irreducible representation is called a factor representation of type I. If every factor representation is type I then $G$ is called type $\boldsymbol{I}$.

Example 2.56. Abelian locally compact groups and compact groups are type I. Moreover, connected nilpotent, exponential, and semisimple Lie groups are type I. (See Führ [33, p. 72] for references.)

The Mautner group is a famous example of a solvable Lie group which is not type I. (See Baggett [10] for details of its representation theory.)

The representation theory of groups of type I enjoys the pleasant features known from abelian locally compact groups and compact groups. For instance, every representation can be uniquely decomposed (in a generalized sense) into irreducible ones. For non-type I groups this is, in general, not true. In that case, to get similar results, one has to restrict attention to type I representations.

Definition 2.57. A representation $\pi$ is called multiplicity-free if $\mathcal{C}(\pi)$ is commutative. $\pi$ is called type $\boldsymbol{I}$ if it is quasi-equivalent to a multiplicity-free representation.

\subsubsection{Direct integral of Hilbert spaces}

Let $M$ be a measurable space and let $\left\{\mathcal{H}_{m}\right\}_{m \in M}$ be a family of nonzero separable Hilbert spaces. Denote the scalar product and norm of $\mathcal{H}_{m}$ by $\langle\cdot, \cdot\rangle_{m}$ and $\|\cdot\|_{m}$, respectively. The vector fields $f \in \prod_{m \in M} \mathcal{H}_{m}$ are considered as functions mapping $m \in M$ to $f(m) \in \mathcal{H}_{m}$. 
Suppose there exists a countable set $\left\{v_{j}\right\}_{j \in \mathbb{N}}$ such that

- the functions $m \mapsto\left\langle v_{j}(m), v_{k}(m)\right\rangle_{a}$ are measurable for all $j, k$,

- and $\left\{v_{j}(m)\right\}_{j \in \mathbb{N}}$ is total in $\mathcal{H}_{m}$ for all $m \in M$.

Then $\left(\left\{\mathcal{H}_{m}\right\}_{m \in M},\left\{v_{j}\right\}_{j \in \mathbb{N}}\right)$ is called a measurable field of Hilbert spaces. A vector field $f$ is called measurable if $m \mapsto\left\langle v_{j}(m), f(m)\right\rangle_{m}$ is measurable for all $j \in \mathbb{N}$. The set of measurable vector fields forms a vector space over the complex numbers.

Even if the family $\left\{v_{j}\right\}_{j \in \mathbb{N}}$ is chosen very complicated, there exists a family $\left\{e_{j}\right\}_{j \in \mathbb{N}}$ which has a particularly easy form and defines the same measurable structure.

Proposition 2.58. Let $\left(\left\{\mathcal{H}_{m}\right\}_{m \in M},\left\{v_{j}\right\}_{j \in \mathbb{N}}\right)$ be a measurable field of Hilbert spaces.

- There exist measurable vector fields $\left\{e_{j}\right\}_{j \in \mathbb{N}}$ such that

$$
\left\{e_{j}(m)\right\}_{j=1, \ldots, \operatorname{dim} \mathcal{H}_{m}}
$$

is a complete orthonormal system and $e_{j}(m)=0$ for $j>\operatorname{dim} \mathcal{H}_{m}$.

- $\left\{v_{j}\right\}_{j \in \mathbb{N}}$ and $\left\{e_{j}\right\}_{j \in \mathbb{N}}$ define the same measurable structure on $\left\{\mathcal{H}_{m}\right\}_{m \in M}$ in the sense that a vector field $f$ is measurable (with respect to $\left\{v_{j}\right\}_{j \in \mathbb{N}}$ ) if and only if $m \mapsto\left\langle e_{j}(m), f(m)\right\rangle_{m}$ is measurable for all $j \in \mathbb{N}$.

- If $f, g$ are measurable vector fields then $m \mapsto\langle f(m), g(m)\rangle_{m}$ is measurable.

This result basically follows from the fact that pointwise orthogonalization by the GramSchmidt process can be realized without affecting measurability. A detailed proof can be found in Folland [31, Prop. 7.19].

Given a measurable field of Hilbert spaces $\left(\left\{\mathcal{H}_{m}\right\}_{m \in M},\left\{v_{j}\right\}_{j \in \mathbb{N}}\right)$ and a $\sigma$-finite measure $\mu$ on $M$ the direct integral of the Hilbert spaces $\mathcal{H}_{m}, m \in M$, denoted by

$$
\int_{M}^{\oplus} \mathcal{H}_{m} \mathrm{~d} \mu(m)
$$

is the set of all measurable vector fields $f$ satisfying

$$
\|f\|^{2}=\int_{M}\|f(m)\|_{m}^{2} \mathrm{~d} \mu(m)<\infty
$$

where two measurable vector fields $f, g$ are identified if $\|f-g\|=0$.

Proposition 2.59. $\int_{M}^{\oplus} \mathcal{H}_{m} \mathrm{~d} \mu(m)$ is a Hilbert space with respect to the scalar product

$$
\langle f, g\rangle:=\int_{M}\langle f(m), g(m)\rangle_{m} \mathrm{~d} \mu(m),
$$

for all $f, g \in \int_{M}^{\oplus} \mathcal{H}_{m} \mathrm{~d} \mu(m)$. 
For sure, the space $\int_{M}^{\oplus} \mathcal{H}_{m} \mathrm{~d} \mu(m)$ depends on the choice of $\left\{v_{j}\right\}_{j \in \mathbb{N}}$ and one can easily find another family $\left\{w_{j}\right\}_{j \in \mathbb{N}}$ which defines a different measurable structure. For instance, by changing $\left\{v_{j}\right\}_{j \in \mathbb{N}}$ on a subset of $M$ which is not measurable. However, it can be shown that there exists a unitary isomorphism between the two Hilbert spaces obtained from $\left\{v_{j}\right\}_{j \in \mathbb{N}}$ and $\left\{w_{j}\right\}_{j \in \mathbb{N}}$ which respects the direct integral structure. (For details see Folland [31, Chp. 7.4].) In that sense, it is not important which measurable structure is used to construct the direct integral of Hilbert spaces. Hence, they are often used without mentioning a measurable structure.

Next, we focus on operators on $\int_{M}^{\oplus} \mathcal{H}_{m} \mathrm{~d} \mu(m)$. A field of operators $\{T(m)\}_{m \in M}$ is called measurable if $\{T(m) f(m)\}_{m \in M}$ is measurable for all measurable vector fields $f$. Measurability can also be characterized as follows.

Proposition 2.60. A field of operators $\{T(m)\}_{m \in M}$ is measurable if and only if $m \mapsto$ $\left\langle v_{j}(m), T(m) v_{k}(m)\right\rangle_{m}$ is measurable for all $j, k \in \mathbb{N}$.

Let $\mu$ be a $\sigma$-finite measure on $M$ and let $T=\{T(m)\}_{m \in M}$ be a measurable field of operators. $T$ is called essentially bounded (i.e., up to null sets) if

$$
\|T\|_{\infty}:=\operatorname{ess~sup}_{m \in M}\|T(m)\|_{\mathrm{op}}<\infty
$$

where $\|\cdot\|_{\text {op }}$ is the operator norm.

Proposition 2.61. Let $T=\{T(m)\}_{m \in M}$ be an essentially bounded measurable field of operators. The operator $\int_{M}^{\oplus} T(m) \mathrm{d} \mu(m)$ on $\int_{M}^{\oplus} \mathcal{H}_{m} \mathrm{~d} \mu(m)$ defined by

$$
\left(\int_{M}^{\oplus} T(m) \mathrm{d} \mu(m) f\right)(m):=T(m) f(m)
$$

is bounded and $\left\|\int_{M}^{\oplus} T(m) \mathrm{d} \mu(m)\right\|_{\mathrm{op}}=\|T\|_{\infty}$.

If $T_{1}=\left\{T_{1}(m)\right\}_{m \in M}$ and $T_{2}=\left\{T_{2}(m)\right\}_{m \in M}$ are essentially bounded measurable fields of operators such that $\int_{M}^{\oplus} T_{1}(m) \mathrm{d} \mu(m)=\int_{M}^{\oplus} T_{2}(m) \mathrm{d} \mu(m)$ then $T_{1}(m)=T_{2}(m) \mu$ almost everywhere.

The operator $\int_{M}^{\oplus} T(m) \mathrm{d} \mu(m)$ is called the direct integral of the field of operators $T$. The space of all integral operators is denoted by

$$
\mathcal{B}^{\oplus}(M, \mu)=\int_{M}^{\oplus} \mathcal{B}\left(\mathcal{H}_{m}\right) \mathrm{d} \mu(m)
$$

and forms a von Neumann algebra contained in $\mathcal{B}\left(\int_{M}^{\oplus} \mathcal{H}_{m} \mathrm{~d} \mu(m)\right)$. Its commutant $\mathcal{B}^{\oplus}(M, \mu)^{\prime}$ consists of all diagonal operators, i.e., the operators

$$
\int_{M}^{\oplus} T(m) \mathrm{d} \mu(m) \in \mathcal{B}^{\oplus}(M, \mu)
$$


of the form

$$
T(m)=t(m) \cdot \mathbb{1}_{\mathcal{H}_{m}}, \quad \text { almost everywhere, }
$$

for some essentially bounded measurable function $t \in L^{\infty}(M, \mu)$.

For a Hilbert space $\mathcal{H}$, let $\mathcal{B}_{1}(\mathcal{H})$ be the space of trace-class operators and let $\mathcal{B}_{2}(\mathcal{H})$ be the space of Hilbert-Schmidt operators on $\mathcal{H}$. Denote by

$$
\mathcal{B}_{1}^{\oplus}(M, \mu) \subseteq \mathcal{B}^{\oplus}(M, \mu)
$$

the Banach space of all direct integrals $T=\int_{M}^{\oplus} T(m) \mathrm{d} \mu(m) \in \mathcal{B}^{\oplus}(M, \mu)$ with $T(m) \in$ $\mathcal{B}_{1}\left(\mathcal{H}_{m}\right)$ almost everywhere and

$$
\|T\|_{1}:=\int_{M}\|T(m)\|_{m, 1} \mathrm{~d} \mu(m)<\infty
$$

where $\|\cdot\|_{m, 1}$ denotes the trace-norm of $\mathcal{B}_{1}\left(\mathcal{H}_{m}\right)$. Likewise, let

$$
\mathcal{B}_{2}^{\oplus}(M, \mu) \subseteq \mathcal{B}^{\oplus}(M, \mu)
$$

be the direct integral of the Hilbert spaces $\left\{\mathcal{B}_{2}\left(\mathcal{H}_{m}\right)\right\}_{m \in M}$. That is the Hilbert space of all operators $T=\int_{M}^{\oplus} T(m) \mathrm{d} \mu(m) \in \mathcal{B}^{\oplus}(M, \mu)$ with $T(m) \in \mathcal{B}_{2}\left(\mathcal{H}_{m}\right)$ almost everywhere satisfying

$$
\|T\|_{2}^{2}:=\int_{M}\|T(m)\|_{m, 2}^{2} \mathrm{~d} \mu(m)<\infty,
$$

where $\|\cdot\|_{m, 2}$ denotes the Hilbert-Schmidt-norm of $\mathcal{B}_{2}\left(\mathcal{H}_{m}\right)$.

Let $G$ be a second countable locally compact group and let $\left\{\left(\pi_{m}, \mathcal{H}_{m}\right)\right\}_{m \in M}$ be a family of representations. If for all $a \in G$ the fields of operators $\left\{\pi_{m}(a)\right\}_{m \in M}$ are measurable, then $\left\{\left(\pi_{m}, \mathcal{H}_{m}\right)\right\}_{m \in M}$ is called a measurable field of representations. In that case, $\int_{M}^{\oplus} \pi_{m} \mathrm{~d} \mu(m)$ given by

$$
\left(\int_{M}^{\oplus} \pi_{m} \mathrm{~d} \mu(m)\right)(a)=\int_{M}^{\oplus} \pi_{m}(a) \mathrm{d} \mu(m)
$$

defines a unitary representation of $G$ on $\int_{M}^{\oplus} \mathcal{H}_{m} \mathrm{~d} \mu(m)$. The representation

$$
\int_{M}^{\oplus} \pi_{m} \mathrm{~d} \mu(m)
$$

is called the direct integral of the representations $\left\{\pi_{m}\right\}_{m \in M}$.

\subsubsection{Decomposition of representations}

The results of Chp. 2.3.1 and Chp. 2.3.2 can be used to decompose a representation as a direct integral. 
Theorem 2.62. Let $G$ be a second countable locally compact group, $\pi$ a unitary representation of $G$ on a separable Hilbert space $\mathcal{H}$, and $B$ a commutative von Neumann subalgebra of $\mathcal{C}(\pi)$.

There exists a standard Borel space $M$, a measurable field $\left\{\mathcal{H}_{m}\right\}_{m \in M}$ of Hilbert spaces, a measurable field $\left\{\pi_{m}\right\}_{m \in M}$ of representations of $G$, and a unitary map $U: \mathcal{H} \rightarrow$ $\int_{M}^{\oplus} \mathcal{H}_{m} \mathrm{~d} \mu(m)$, such that

- $U \pi(a) U^{-1}=\int_{M}^{\oplus} \pi_{m}(a) \mathrm{d} \mu(m)$ for all $a \in G$

- and $U B U^{-1}$ is the algebra of diagonal operators on $\int_{M}^{\oplus} \mathcal{H}_{m} \mathrm{~d} \mu(m)$.

With some extra work Thm. 2.62 can be refined to construct a decomposition into factor representations.

For $d \in \overline{\mathbb{N}}$, let $\mathcal{H}_{d}$ be the Hilbert space of dimension $d$ defined in eq. (2.11) and (2.12) in Chp.2.3.1. Furthermore, let $\operatorname{Rep}_{d}(G)$ be the space of all representations of $G$ on the Hilbert space $\mathcal{H}_{d}$ endowed with the coarsest $\sigma$-algebra making all functions

$$
\pi \mapsto\langle f, \pi(a) g\rangle, \quad \text { for } a \in G, f, g \in \mathcal{H}_{d}
$$

measurable. Let

$$
\operatorname{Rep}(G)=\bigcup_{d \in \overline{\mathbb{N}}} \operatorname{Rep}_{d}(G)
$$

be the space of all representations of $G$. A subset $E \subseteq \operatorname{Rep}(G)$ is measurable if $E \cap$ $\operatorname{Rep}_{d}(G)$ is measurable for all $d \in \overline{\mathbb{N}}$. The set of all factor representations $\operatorname{Fac}(G)$ and the set of all irreducible representations $\operatorname{Irr}(G)$ are measurable and the inclusions

$$
\operatorname{Irr}(G) \hookrightarrow \operatorname{Fac}(G) \hookrightarrow \operatorname{Rep}(G)
$$

are measurable maps (see Dixmier [21, 16.6.1]). The dual $\hat{G}$ and the quasi-dual $\check{G}$ of $G$ are the spaces

$$
\hat{G}=\operatorname{Irr}(G) / \check{G}=\operatorname{Fac}(G) / \approx
$$

endowed with quotient measurable structure, which is called the Mackey Borel structure. Clearly, the inclusion $\hat{G} \hookrightarrow \check{G}$ is measurable. If $G$ is type I, then all factor representations are multiples of irreducible representations and $\check{G}=\hat{G}$. The Mackey Borel structure separates points, i.e., the sets $\{[\sigma]\}$ are measurable for all $[\sigma] \in \check{G}$. In general, $\hat{G}$ and $\check{G}$ are not standard Borel spaces. However, the measures which appear in this thesis will be of the following class.

Definition 2.63. Let $M$ be a measurable space and $\mu$ a measure on $M$. Then $\mu$ is called a standard measure if there exists a measurable subset $X \subseteq M$ such that $X$ is a standard Borel space and $\mu(M \backslash X)=0$.

Now, we are prepared to formulate the following theorem. 
Theorem 2.64. Let $G$ be a second countable locally compact group and $\pi$ a representation of $G$ on a separable Hilbert space.

There exists a standard measure $\mu$ on $\check{G}$, a measurable field of Hilbert spaces $\left\{\mathcal{H}_{p}\right\}_{p \in \check{G}}$, a measurable field of representations $\left\{\pi_{p}\right\}_{p \in \check{G}}$ on $\left\{\mathcal{H}_{p}\right\}_{p \in \check{G}}$ with $\pi_{p} \in p$ for $\mu$-almost every $p \in \check{G}$, and a unitary map $U: \mathcal{H} \rightarrow \int_{\breve{G}}^{\oplus} \mathcal{H}_{p} \mathrm{~d} \mu(p)$, such that

- $U \pi(a) U^{-1}=\int_{\breve{G}}^{\oplus} \pi_{p}(a) \mathrm{d} \mu(p)$ for all $a \in G$

- and the center $U \mathcal{Z}(\pi) U^{-1}=\mathcal{Z}\left(U \mathcal{C}(\pi) U^{-1}\right)$ of $U \mathcal{C}(\pi) U^{-1}$ is the algebra of diagonal operators on $\int_{\breve{G}}^{\oplus} \mathcal{H}_{p} \mathrm{~d} \mu(p)$.

If $\mu^{\prime}$ and $\left\{\pi_{p}^{\prime}\right\}_{p \in \check{G}}$ also have these properties, then $\mu$ is equivalent to $\mu^{\prime}$ and $\pi_{p}$ is equivalent to $\pi_{p}^{\prime}$ for $\mu$-almost every $p$.

The decomposition in Thm. 2.64 is called central decomposition of $\pi$. The measure $\mu$ is referred to as the central measure of $\pi$.

If $\pi$ is a representation of type I, then $\mu$ is supported on $\hat{G}$, i.e., $\mu(\check{G} \backslash \hat{G})=0$. In that case, almost all $\pi_{p}$ are of the form $m_{\pi}(p) \cdot \mathcal{H}_{\sigma_{p}}$, where $\sigma_{p} \in p$ is irreducible, and

$$
\mathcal{H} \cong \int_{\hat{G}}^{\oplus} m_{\pi}(p) \cdot \mathcal{H}_{\sigma_{p}} \mathrm{~d} \mu(p), \quad \pi \cong \int_{\hat{G}}^{\oplus} m_{\pi}(p) \cdot \sigma_{p} \mathrm{~d} \mu(p) .
$$

The map $m_{\pi}: \hat{G} \rightarrow \overline{\mathbb{N}}$ is measurable and counts the multiplicity of an irreducible representation in $\pi$. It is defined up to null sets.

The central decomposition provides a useful tool to characterize the relation of representations.

Theorem 2.65. Let $\pi_{1}, \pi_{2}$ be two representations of $G$ and let

$$
\begin{aligned}
& \pi_{1} \cong \int_{\hat{G}}^{\oplus} \pi_{1, p} \mathrm{~d} \mu_{1}(p), \\
& \pi_{2} \cong \int_{\hat{G}}^{\oplus} \pi_{2, p} \mathrm{~d} \mu_{2}(p)
\end{aligned}
$$

be their central decomposition.

- $\pi_{1}$ is quasi-equivalent to a subrepresentation of $\pi_{2}$ if and only if $\mu_{1}$ is absolutely continuous with respect to $\mu_{2}$.

- $\pi_{1}$ and $\pi_{2}$ are disjoint if and only if $\mu_{1}$ and $\mu_{2}$ are mutually singular.

If both representations are type I, then one can give an even more precise statement.

Theorem 2.66. Let $\pi_{1}, \pi_{2}$ be two type I representations of $G$ and let

$$
\begin{aligned}
& \pi_{1} \cong \int_{\hat{G}}^{\oplus} m_{\pi_{1}}(p) \cdot \sigma_{p} \mathrm{~d} \mu_{1}(p), \\
& \pi_{2} \cong \int_{\hat{G}}^{\oplus} m_{\pi_{2}}(p) \cdot \sigma_{p} \mathrm{~d} \mu_{2}(p)
\end{aligned}
$$


be their central decomposition. Then, $\pi_{1}$ is a subrepresentation of $\pi_{2}$ if and only if $\mu_{1}$ is absolutely continuous with respect to $\mu_{2}$ and

$$
m_{\pi_{1}}(p) \leq m_{\pi_{2}}(p)
$$

almost everywhere.

\subsubsection{Plancherel decomposition}

For the left regular representation Thm. 2.64takes a particular form, which is known as the Plancherel decomposition. Recall that the left regular representation $\left(\lambda_{G}, L^{2}(G)\right)$ and the right regular representation $\left(\rho_{G}, L^{2}(G)\right)$ of $G$, and the two-sided representation $\left(\tau_{G}, L^{2}(G)\right)$ of $G \times G$ are given by

$$
\begin{aligned}
{\left[\lambda_{G}(b) f\right](a) } & =f\left(b^{-1} a\right), \\
{\left[\rho_{G}(b) f\right](a) } & =\Delta(b)^{\frac{1}{2}} f(a b), \\
{\left[\tau_{G}(b, c) f\right](a) } & =\left[\lambda_{G}(b) \rho_{G}(c)\right] f(a)=\Delta(c)^{\frac{1}{2}} f\left(b^{-1} a c\right),
\end{aligned}
$$

for $f \in L^{2}(G)$.

Let $f \in L^{1}(G)$ and let $(\pi, \mathcal{H})$ a representation of $G$. Then there exists a bounded operator, denoted by $\pi(f)$ which satisfies

$$
\langle v, \pi(f) w\rangle=\int_{G} f(a) \cdot\langle v, \pi(a) w\rangle \mathrm{d} a \quad \forall v, w \in \mathcal{H} .
$$

The integral

$$
\pi(f)=\int_{G} f(a) \pi(a) \mathrm{d} a
$$

is said to be weakly convergent.

If $\lambda_{G}$ is type I, then $\rho_{G}, \tau_{G}$ are type I, as well. Moreover, $\tau_{G}$ is multiplicity-free. (See Duflo and Moore [25, Chp. 5] for references.) If $G$ is unimodular, then the central decomposition of $\tau_{G}$ yields the following result, which is known as the Plancherel Theorem for unimodular group.

Theorem 2.67 (Plancherel Theorem (unimodular case)). Let $G$ be a unimodular, second countable, locally compact group and suppose that $\lambda_{G}$ is type I. Then there exists a unique measure $\mu_{G}$ on $\hat{G}$ such that

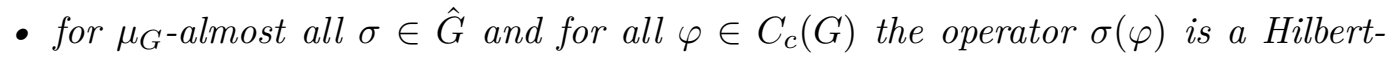
Schmidt operator $\hat{\varphi}(\sigma): \mathcal{H}_{\sigma} \rightarrow \mathcal{H}_{\sigma}$,

- the map $\varphi \mapsto \hat{\varphi}$ extends to a unitary isomorphism $L^{2}(G) \rightarrow \mathcal{B}_{2}^{\oplus}(\hat{G})$ that intertwines the two-sided regular representation $\tau_{G}$ and $\int_{\hat{G}}^{\oplus} \sigma \otimes \bar{\sigma} \mathrm{d} \mu_{G}(\sigma)$. 
Here, we use the convention that for any class $p \in \hat{G}$ we fixed an irreducible representative $\left(\sigma, \mathcal{H}_{\sigma}\right) \in p$ and use the shorthand notation

$$
\int_{\hat{G}}^{\oplus} \sigma \otimes \bar{\sigma} \mathrm{d} \mu_{G}(\sigma)
$$

instead of

$$
\int_{\hat{G}}^{\oplus} \sigma_{p} \otimes \overline{\sigma_{p}} \mathrm{~d} \mu_{G}(p)
$$

with $\sigma_{p} \in p$. Recall that $\mathcal{B}_{2}^{\oplus}(\hat{G})$ is a direct integral of Hilbert spaces

$$
\mathcal{B}_{2}^{\oplus}(\hat{G})=\mathcal{B}_{2}^{\oplus}\left(\hat{G}, \mu_{G}\right)=\int_{\hat{G}}^{\oplus} \mathcal{B}_{2}\left(\mathcal{H}_{\sigma}\right) \mathrm{d} \mu_{G}(\sigma) .
$$

Since the tensor product $\mathcal{H}_{\sigma} \otimes \overline{\mathcal{H}_{\sigma}}$ is nothing but $\mathcal{B}_{2}\left(\mathcal{H}_{\sigma}\right)$, the representation $\sigma \otimes \bar{\sigma}$ acts on $\mathcal{B}_{2}\left(\mathcal{H}_{\sigma}\right)$ by

$$
[\sigma(b) \otimes \overline{\sigma(c)}] T=\sigma(b) T \sigma(c)^{*}, \quad \forall b, c \in G, T \in \mathcal{B}_{2}\left(\mathcal{H}_{\sigma}\right) .
$$

In the nonunimodular case, we have $(\tau(b, c) \varphi)(a)=\Delta_{G}(c)^{1 / 2} \varphi\left(b^{-1} a c\right)$ and, therefore $\sigma(\tau(b, c) \varphi)=\Delta(c)^{1 / 2} \sigma(b) \sigma(\varphi) \sigma(c)^{*}$. Due to the factor $\Delta_{G}(c)^{1 / 2}$, the map $\varphi \mapsto(\sigma \mapsto$ $\sigma(\varphi)$ ) has no chance to extend to an intertwiner between $\tau$ and $\sigma \otimes \bar{\sigma}$. (In fact, $\sigma(\varphi)$ even does not necessarily define a Hilbert-Schmidt operator.) To solve this problem one introduces an operator $D_{\sigma}: \mathcal{H}_{\sigma} \rightarrow \mathcal{H}_{\sigma}$ with the property that $\sigma(c) D_{\sigma}=\Delta_{G}(c)^{1 / 2} D_{\sigma} \sigma(c)$ in order to obtain

$$
\sigma(\tau(b, c) \varphi) D_{\sigma}=\Delta(c)^{1 / 2} \sigma(b) \sigma(\varphi) \sigma(c)^{*} D_{\sigma}=\sigma(b) \sigma(\varphi) D_{\sigma} \sigma(c)^{*}
$$

In [25] Duflo and Moore showed that these operators exist and they gave a precise description.

Lemma 2.68. Let $H \leq \operatorname{ker}\left(\Delta_{G}\right)$ and assume that $(\pi, \mathcal{H})$ is a unitary representation of $G$ induced from $H$ (see Variant 1 in Chp. 2.2.2). Then,

$$
\left(D_{\pi} f\right)(a)=\Delta_{G}(a)^{1 / 2} f(a)
$$

defines an operator $\mathcal{H} \rightarrow \mathcal{H}$. It is a densely defined, selfadjoint, positive operator with a densely defined inverse, and satisfies the relation

$$
\pi(a)^{*} D_{\pi} \pi(a)=\Delta_{G}(a)^{1 / 2} D_{\pi}
$$

Together with Lem.2.68, the Plancherel Theorem in the general case can be formulated as follows. 
Theorem 2.69 (Plancherel Theorem (general case)). Let $G$ be a second countable locally compact group and suppose that $\lambda_{G}$ is type $I$. Then, there exists a unique measure $\mu_{G}$ on $\hat{G}$ such that

- $\mu$-almost all $\sigma \in \hat{G}$ are induced from a representation of $\operatorname{ker} \Delta_{G}$,

- for $\mu$-almost all $\pi$ and for all $\varphi \in C_{c}(G)$ the operator $\sigma(\varphi) D_{\sigma}$ extends to a Hilbert-Schmidt operator $\hat{\varphi}(\sigma): \mathcal{H}_{\sigma} \rightarrow \mathcal{H}_{\sigma}$,

- and the map $\varphi \mapsto \hat{\varphi}$ extends to a unitary isomorphism $\mathcal{P}: L^{2}(G) \rightarrow \mathcal{B}_{2}^{\oplus}(\hat{G})$ that intertwines the two-sided regular representation $\tau$ and $\int_{\hat{G}}^{\oplus} \sigma \otimes \bar{\sigma} \mathrm{d} \mu(\sigma)$.

The measure $\mu_{G}$ is called the Plancherel measure and the isometric isomorphism $\mathcal{P}: L^{2}(G) \rightarrow \mathcal{B}_{2}^{\oplus}(\hat{G})$ is called Plancherel transformation. The Plancherel transform of a functions $f \in L^{2}(G)$ is denoted by

$$
\mathcal{P}(f)=\hat{f} .
$$

For $f, g \in L^{2}(G)$, the formula

$$
\langle f, g\rangle=\int_{G} \overline{f(a)} g(a) \mathrm{d} a=\int_{\hat{G}} \operatorname{Tr}_{\sigma}\left[\hat{f}(\sigma)^{*} \hat{g}(\sigma)\right] \mathrm{d} \mu_{G}(\sigma)
$$

is called Plancherel formula.

If $G$ is unimodular, then $D_{\sigma}$ is (a multiple of) the identity operator. If $G$ is nonunimodular, then $D_{\sigma}$ is an unbounded operator as $\Delta_{G}$ is unbounded. In particular, we get the following result.

Corollary 2.70. If $G$ is nonunimodular, then $\mu_{G}$-almost all irreducible representations of $G$ are infinite-dimensional. Moreover, the multiplicity $m_{\lambda_{G}}(\sigma)$ of almost all irreducible representations $\sigma$ in the left regular representation $\lambda_{G}$ is $\infty$.

Let $f \in L^{1}(G) \cap L^{2}(G), g \in L^{2}(G)$ and suppose that $\hat{g}(\sigma) D_{\sigma} \in \mathcal{B}_{1}\left(\mathcal{H}_{\sigma}\right)$ almost everywhere. Then, the Plancherel transformation $\mathcal{P}$ can be written as

$$
\mathcal{P}(f)(\sigma)=\hat{f}(\sigma)=\left[\int_{G} f(a) \sigma(a) \mathrm{d} a\right] D_{\sigma}
$$

and Plancherel inversion $\mathcal{P}^{-1}$ can be written as

$$
\mathcal{P}^{-1}(\hat{g})(a)=\int_{\hat{G}} \operatorname{Tr}_{\sigma}\left[\hat{g}(\sigma) D_{\sigma} \sigma(a)^{*}\right] \mathrm{d} \mu_{G}(\sigma) .
$$

Eq. 2.15 follows by definition and eq. 2.16 follows from the Plancherel formula eq. 2.14.

Another consequence of Thm. 2.69 is that the von Neumann algebra $\mathcal{C}\left(\lambda_{G}\right)$ is isomorphic to $\mathcal{B}^{\oplus}(\hat{G})$. In particular, it has the following form. 
Theorem 2.71. With the assumptions of Thm. 2.69, for all $T \in \mathcal{C}\left(\lambda_{G}\right)$ there exists a direct integral operator $\int_{\hat{G}} \hat{T}(\sigma) \mathrm{d} \mu_{G}(\sigma) \in \mathcal{B}^{\oplus}(\hat{G})$ such that

$$
\widehat{T f}(\sigma)=\hat{f}(\sigma) \hat{T}(\sigma) .
$$

The $\operatorname{map} \mathcal{C}\left(\lambda_{G}\right) \rightarrow \mathcal{B}^{\oplus}(\hat{G})$ is an isometric isomorphism.

\subsubsection{Remarks on the Plancherel Theorem}

On the one hand, in [33, Thm. 3.48] Führ requires that $G$ and $N=$ ker $\Delta_{G}$ are type I and $N$ is regularly embedded in the following sense.

Definition 2.72. Let $G$ be a locally compact group and suppose that $G$ is second countable. Let $N$ be closed normal subgroup of $G$.

- Let $\sigma$ be an irreducible representation of $N$ and let $a \in G$. Then a. $\sigma$, given by

$$
a . \sigma(n)=\sigma\left(a^{-1} n a\right) \quad \forall n \in N
$$

defines an irreducible representation of $N$. The map $G \times \hat{N} \rightarrow \hat{N},(a, \sigma) \mapsto a . \sigma$, defines an action of $G$ on $\hat{N}$.

- $\hat{N} / G$ is $\mu_{N}$-standard if there exists a conull $G$-invariant subset $X \subseteq \hat{N}$ such that $X / G$ endowed with quotient measurable structure is standard. $\left(\mu_{N}\right.$ is the Plancherel measure of $N$.)

- $G$ acts regularly on $\hat{N}$ if there exists a conull $G$-invariant subset $X \subseteq \hat{N}$ and a countable collection $\left\{E_{n}\right\}_{n \in \mathbb{N}}$ of $G$-invariant measurable subsets of $\hat{N}$ such that every orbit $\mathcal{O}$ in $\hat{N}$ satisfies

$$
\mathcal{O}=\bigcap\left\{E_{n} \mid n \in \mathbb{N}, \mathcal{O} \subseteq E_{n}\right\}
$$

In that case $N$ is said to be regularly embedded.

On the other hand, Folland requires in [31] only that $N=\operatorname{ker} \Delta_{G}$ is type I and regularly embedded in $G$.

In [25, Thm. 5] Duflo and Moore showed that it is sufficient that $\lambda_{G}$ is type I. (To be more precise, they showed the Plancherel Theorem for the type I part of $\lambda_{G}$.) The relation to the statements given by Führ and Folland comes from the following results.

Theorem 2.73. Let $G$ be a locally compact group and suppose that $G$ is second countable and nonunimodular. Let $N=\operatorname{ker} \Delta_{G}$. Then the following are equivalent.

(i) $\lambda_{N}$ is type I and $\hat{N} / G$ is $\nu_{N}$-standard.

(ii) $\lambda_{N}$ is type $I$ and $G$ acts $\nu_{N}$-regularly on $\hat{N}$.

(iii) $\lambda_{G}$ is type $I$. 
Proof.

- The implication $(i) \Rightarrow(i i)$ follows as standard Borel spaces are countably separated.

- $($ ii $) \Rightarrow($ iii $)$ was shown by Tatsuuma in [58, Thm. 5.1].

- $($ iii $) \Rightarrow(i)$ is Cor. 1 of Thm. 6 [25] by Duflo and Moore.

Corollary 2.74. Let $G$ be a locally compact group and suppose that $G$ is second countable and nonunimodular. Let $N=\operatorname{ker} \Delta_{G}$.

Each of the following conditions is sufficient for $\lambda_{G}$ to be type $I$.

- $G$ is type $I$.

- $N$ is type I and $\hat{N} / G$ is standard.

- $N$ is type $I$ and $G$ acts regularly on $\hat{N}$.

In particular, if one of those conditions is fulfilled then the Plancherel Theorem can be applied.

However, Thm. 2.73 only applies to $\operatorname{ker}\left(\Delta_{G}\right)$ and not to general normal closed subgroups. If $G$ is type I then a closed normal subgroup of $G$ need not necessarily be regularly embedded as Auslander and Moore showed in a counterexample represented in [9, Sec. III.5].

\subsection{Contractions of Lie algebras, Lie groups, and repre- sentation}

The concept of contraction comes from physics and goes back to Segal [57] and Inönü and Wigner [42]. The motivation is that it should be possible to recover classical physics from advanced theories (quantum physics and general relativity) in the socalled classical limit. In that sense physicists want to obtain the Galilei group, which is the symmetry group of classical mechanics, as a limit of the Poincaré group, which is the symmetry group of relativistic mechanics. Furthermore, the Schrödinger representations, describing quantum mechanics, should be the classical (nonrelativistic) limit of representations of the Poincaré group, which describe relativistic quantum mechanics. In [42] Inönü and Wigner give a suitable construction to understand this kind of limit of groups and their representations. In [23] and 24] Dooley and Rice formulated contraction in a rigorous way.

During the last decade the notion of group contractions developed and many authors worked on that field. The following is an excerpt of the overview article [18 by Cahen. Let $G, G_{0}$ be two real Lie groups of the same dimension $n$ and denote their Lie algebras by $(\mathfrak{g},[\cdot, \cdot]),\left(\mathfrak{g}_{0},[\cdot, \cdot]_{0}\right)$. 


\section{Definition 2.75.}

- A (Lie algebra) contraction of $\mathfrak{g}$ to $\mathfrak{g}_{0}$ is a family of linear isomorphisms $\left(c_{r}\right)_{r \in(0,1]}$ from $\mathfrak{g}_{0}$ to $\mathfrak{g}$ such that

$$
\lim _{r \rightarrow 0} c_{r}^{-1}\left(\left[c_{r}(X), c_{r}(Y)\right]\right)=[X, Y]_{0}, \quad \forall X, Y \in \mathfrak{g}_{0} .
$$

- $A$ (Lie group) contraction of $G$ to $G_{0}$ is a family of smooth maps $\left(C_{r}\right)_{r \in(0,1]}$ from an open neighborhood $V$ of the identity element $e_{0} \in G_{0}$ to $G$ such that

(i) $C_{r}\left(e_{0}\right)=e$ for every $r \in(0,1]$;

(ii) There exists an open neighborhood $W \subseteq G$ of e such that $C_{r}$ is a diffeomorphism from $C_{r}^{-1}\left(W^{2}\right)$ to $W^{2}$ for all $r \in(0,1]$;

(iii) for each $x \in V$ there exists an $r_{x} \in(0,1]$ such that $C_{r}(x) \in W$ for all $r<r_{x}$;

(iv) and for all $x, y \in V$,

$$
\lim _{r \rightarrow 0} C_{r}^{-1}\left(C_{r}(x) C_{r}(y)\right)=x y
$$

From the definition of the Lie group contraction it follows that the existence of a contraction is a local property and it does not depend on the global structure of $G$ and $G_{0}$. Hence, it is not very surprising that there is a close relation between Lie group contractions and the contraction of their Lie algebras.

\section{Lemma 2.76.}

- If $\left(C_{r}\right)_{r \in(0,1]}$ is a contraction of $G$ to $G_{0}$ then $\left(\mathrm{d} C_{r}\right)_{r \in(0,1]}$ is contraction of $\mathfrak{g}$ to $\mathfrak{g}_{0}$.

- If $\left(c_{r}\right)_{r \in(0,1]}$ is contraction of $\mathfrak{g}$ to $\mathfrak{g}_{0}$ and the family $\left(\left\|c_{r}\right\|_{\mathrm{op}}\right)_{r \in(0,1]}$ is bounded then $\left(C_{r}\right)_{r \in(0,1]}$ defined by

$$
C_{r}=\exp _{G} \circ c_{r} \circ \exp _{G_{0}}^{-1}
$$

is a contraction of $G$ to $G_{0}$.

Given a contraction $\left(C_{r}\right)_{r \in(0,1]}$ of $G$ to $G_{0}$ one can study the relationship between representations of $G$ and $G_{0}$.

Definition 2.77. Let $(\pi, \mathcal{H})$ be a representation of $G_{0}$. A family $\left(\left(\pi_{n}, \mathcal{H}_{n}\right)\right)_{n \in \mathbb{N}}$ of representations of $G$ contracts to $\pi$ if there exists a sequence $r(n) \in(0,1]$ converging to 0 , a family of unitary operators $A_{n}: \mathcal{H}_{n} \rightarrow \mathcal{H}$, and a dense subspace $\mathcal{D} \subseteq \mathcal{H}$ such that

(i) for each $f \in \mathcal{D}$ there exists $n_{f} \in \mathbb{N}$ such that for all $n \geq n_{f}, f \in A_{n}\left(\mathcal{H}_{n}\right)$;

(ii) and for all $f \in \mathcal{D}$ and $x \in V$,

$$
\lim _{n \rightarrow \infty}\left\|A_{n} \pi_{n}\left(C_{r(n)}(x)\right) A_{n}^{-1} f-\pi(x) f\right\|_{\mathcal{H}}=0 .
$$


A special case of the contraction procedure defined in Def. 2.75 is the contraction along a subalgebra or subgroup, respectively (cf. Antoine and Vandergheynst [6]). Let $G$ be a connected Lie group, $H \leq G$ a closed subgroup, and denote their Lie algebras by $\mathfrak{g}$ and $\mathfrak{h}$. Then $\mathfrak{h}$ is a linear subspace of $\mathfrak{g}$ and there exists a complementary vector space $\mathfrak{v} \subseteq \mathfrak{g}$ such that $\mathfrak{g}=\mathfrak{v} \oplus \mathfrak{h}$. For $X \in \mathfrak{g}$ write $X=X_{\mathfrak{v}}+X_{\mathfrak{h}}$, where $X_{\mathfrak{v}} \in \mathfrak{v}$ and $X_{\mathfrak{h}} \in \mathfrak{h}$. The linear maps $c_{r}: \mathfrak{g} \rightarrow \mathfrak{g}, r \in(0,1]$,

$$
c_{r}\left(X_{\mathfrak{v}}+X_{\mathfrak{h}}\right)=r X_{\mathfrak{v}}+X_{\mathfrak{h}}
$$

define a contraction of $\mathfrak{g}$ to $\mathfrak{g}_{0}$, where $\mathfrak{g}_{0}=\mathfrak{v} \oplus \mathfrak{h}$, defined as a vector space, with the Lie bracket

$$
\left[X_{\mathfrak{v}}+X_{\mathfrak{h}}, Y_{\mathfrak{v}}+Y_{\mathfrak{h}}\right]_{0}=\left(\left[X_{\mathfrak{h}}, Y_{\mathfrak{v}}\right]+\left[X_{\mathfrak{v}}, Y_{\mathfrak{h}}\right]\right)_{\mathfrak{v}}+\left[X_{\mathfrak{h}}, Y_{\mathfrak{h}}\right]
$$

where $X_{\mathfrak{v}}, Y_{\mathfrak{v}} \in \mathfrak{v}, X_{\mathfrak{h}}, Y_{\mathfrak{h}} \in \mathfrak{h}$. As can be seen from eq. 2.18) $\mathfrak{v}$ is an abelian subalgebra of $\mathfrak{g}_{0}$ and $\mathfrak{h}$ acts on $\mathfrak{v}$. Hence, $\mathfrak{g}_{0}$ is semidirect product of the form $\mathfrak{g}_{0}=\mathfrak{v} \rtimes \mathfrak{h}$.

The simply connected, connected Lie group corresponding to $\mathfrak{v}$ is $(V,+)=(\mathfrak{v},+)$. The action of $\mathfrak{h}$ on $\mathfrak{v}$ can be lifted to an action of $H$ on $\mathfrak{v}$ and by identifying $\mathfrak{v}$ with $V$ (via the exponential map $\exp _{V}: \mathfrak{v} \rightarrow V$ ) the latter induces an action of $H$ on $V$. Therefore, it seems natural to set $G_{0}=V \rtimes H$ and indeed the maps $C_{r}: G_{0} \rightarrow G, r \in(0,1]$,

$$
C_{r}\left(\exp _{V}\left(X_{\mathfrak{v}}\right), h\right)=\exp _{G}\left(r X_{\mathfrak{v}}\right) h, \quad \exp _{V}\left(X_{\mathfrak{v}}\right) \in V, h \in H,
$$

define a contraction of $G$ to $G_{0}$.

Definition 2.78. Let $G$ be a connected Lie group and $H$ a closed subgroup. Denote their Lie algebras by $\mathfrak{g}$ and $\mathfrak{h}$. Let $\mathfrak{v}$ be the linear complement of $\mathfrak{h}$ in $\mathfrak{g}$ and consider $\mathfrak{v}$ as an abelian Lie algebra with simply connected, connected abelian Lie group $V$.

- The contraction of $\mathfrak{g}$ along $\mathfrak{h}$ is the Lie algebra

$$
\mathfrak{g}_{0}=\mathfrak{v} \rtimes \mathfrak{h}
$$

with the Lie bracket given by eq. 2.18), together with the family of maps $\left(c_{r}: \mathfrak{g}_{0} \rightarrow\right.$ $\mathfrak{g})_{r \in(0,1]}$ defined by eq. 2.17).

- The contraction of $G$ along $H$ is the Lie group

$$
G_{0}=V \rtimes H
$$

together with the family of maps $\left(C_{r}: G_{0} \rightarrow G\right)_{r \in(0,1]}$ defined by eq. (2.19).

Here, it becomes apparent why the construction is called contraction. When $r$ goes to 0 the elements of $\mathfrak{v}$ or, to be more precise, the corresponding structure constants of $\mathfrak{g}$, are contracted and shrink to 0 . 


\section{CHAPTER 3}

\section{Continuous wavelet transformations and admissibility conditions}

The aim of this chapter is to give an overview over the different approaches to (generalized) continuous wavelet transformations. This includes a collection of different constructions as well as a systematic analysis with group-theoretical methods. These constructions will be used as a starting point for new approaches.

\subsection{Continuous wavelet transformations and generaliza- tions}

Motivated by the wide field of applications, there is a large number of constructions which were developed to extend the continuous wavelet transformation. In the following, we select the most important constructions. The approaches which will be presented within this section are just a small compilation. A more complete list of references can be found in the textbook [3] by Ali, Antoine, and Gazeau.

\subsubsection{Classical continuous wavelet transformation}

From Fourier analysis it is known that any function $f \in L^{2}(\mathbb{R})$ can be written in terms of plane waves $\chi_{k}(x)=e^{-2 \pi i k x}$. Likewise, one can ask whether it is possible to express $f$ in terms of waves which are similar to a given localized wavelet $\psi \in L^{2}(\mathbb{R})$, i.e., by functions $\psi_{b, a} \in L^{2}(\mathbb{R})$ of the form

$$
\psi_{b, a}(x)=|a|^{-\frac{1}{2}} \psi\left(a^{-1}(x-b)\right)
$$

for $a, b \in \mathbb{R}, a \neq 0$. The parameter $a$ describes the dilation of $\psi$ whereas the parameter $b$ describes the translation. The factor $|a|^{-\frac{1}{2}}$ makes sure that $\left\|\psi_{b, a}\right\|_{2}=\|\psi\|_{2}$.

The answer to that question is positive. For suitable functions $\psi \in L^{2}(\mathbb{R})$ one can show that the map

$$
V_{\psi}: L^{2}(\mathbb{R}) \rightarrow L^{2}\left(\mathbb{R} \times \mathbb{R}_{\neq 0},|a|^{-2} \mathrm{~d} b \mathrm{~d} a\right), \quad V_{\psi} f(b, a)=\left\langle\psi_{b, a}, f\right\rangle,
$$


is an isometry. Its adjoint $V_{\psi}^{*}: L^{2}\left(\mathbb{R} \times \mathbb{R}_{\neq 0},|a|^{-2} \mathrm{~d} b \mathrm{~d} a\right) \rightarrow L^{2}(\mathbb{R})$,

$$
V_{\psi}^{*} F=\int_{\mathbb{R} \times \mathbb{R} \neq 0} \psi_{b, a} \cdot F(b, a)|a|^{-2} \mathrm{~d} b \mathrm{~d} a,
$$

converging in the weak sense, is surjective and yields the decomposition

$$
f=\int_{\mathbb{R} \times \mathbb{R} \neq 0} \psi_{b, a} \cdot V_{\psi} f(b, a)|a|^{-2} \mathrm{~d} b \mathrm{~d} a .
$$

The transformation $V_{\psi}$ is known as the (classical) continuous wavelet transformation.

It is due to Grossmann, Morlet and Paul [39] who noticed (based on the survey by Aslaksen and Klauder [7]) that the classical continuous wavelet transformation is deeply connected to the affine group $G_{\text {aff }}(\mathbb{R})$ of the real line, which is given by

$$
G_{\text {aff }}(\mathbb{R})=\mathbb{R} \rtimes \mathbb{R}_{\neq 0}=\left\{(b, a) \mid a \in \mathbb{R}_{\neq 0}, b \in \mathbb{R}\right\} .
$$

Its group law and inversion are given by

$$
(b, a)\left(b^{\prime}, a^{\prime}\right)=\left(a b^{\prime}+b, a a^{\prime}\right), \quad(b, a)^{-1}=\left(-a^{-1} b, a^{-1}\right)
$$

for $(b, a),\left(b^{\prime}, a^{\prime}\right) \in G_{\mathrm{aff}}(\mathbb{R})$. As the left and right Haar measure have the form

$$
\mathrm{d}(b, a)=|a|^{-2} \mathrm{~d} a \mathrm{~d} b, \quad \mathrm{~d}(b, a)^{-1}=|a|^{-1} \mathrm{~d} a \mathrm{~d} b,
$$

where $\mathrm{d} a, \mathrm{~d} b$ denotes the Lebesgue measure on $\mathbb{R}_{\neq 0} \subseteq \mathbb{R}$ and $\mathbb{R}$, it is a nonunimodular group with modular function $\Delta(b, a)=|a|$. Its action on $\mathbb{R}$, given by

$$
(b, a) x=a x+b \quad \text { for }(b, a) \in G_{\mathrm{aff}}(\mathbb{R}), x \in \mathbb{R},
$$

induces a unitary representation $\pi$ of $G_{\text {aff }}(\mathbb{R})$ on the Hilbert space $L^{2}(\mathbb{R})$, which has the form

$$
[\pi(b, a) f](x)=|a|^{-\frac{1}{2}} f\left(a^{-1}(x-b)\right)=f_{b, a}(x)
$$

The coefficient functions of $\pi$ have the form

$$
C_{f} g(b, a):=\langle\pi(b, a) f, g\rangle=\int_{\mathbb{R}}|a|^{-\frac{1}{2}} \overline{f\left(a^{-1}(x-b)\right)} g(x) \mathrm{d} x=\left(f_{a}^{*} * g\right)(b),
$$

where $f_{a}^{*} \in L^{2}(\mathbb{R})$ is given by

$$
f_{a}^{*}(x)=|a|^{-\frac{1}{2}} \overline{f\left(-a^{-1} x\right)}
$$


and the convolution $f * g$ is defined by

$$
(f * g)(x)=\int_{\mathbb{R}} f(x-y) g(y) \mathrm{d} y
$$

whenever the integral in eq. (3.1) is well-defined.

Recall that the Plancherel transform of function $f \in L^{1}(\mathbb{R}) \cap L^{2}(\mathbb{R})$ has the form

$$
\hat{f}(k)=\int_{\mathbb{R}} f(x) e^{2 \pi i k x} \mathrm{~d} k \quad \forall k \in \mathbb{R} .
$$

Using this convention the Plancherel formula and the Plancherel transform of a convolution are given by

$$
\|f\|_{2}^{2}=\|\hat{f}\|_{2}^{2}:=\int_{\mathbb{R}}|\hat{f}(k)|^{2} \mathrm{~d} k, \quad \text { and } \quad \widehat{f * g}=\hat{f} \cdot \hat{g} .
$$

Suppose that $C_{f} g \in L^{2}\left(G_{\text {aff }}(\mathbb{R})\right)$. Then the functions $b \mapsto C_{f} g(b, a)$ are in $L^{2}(\mathbb{R})$ for almost all $a \neq 0$ and we can verify that their Plancherel transforms have the form

$$
\widehat{f_{a}^{*} * g}(k)=\hat{f}_{a}^{*}(k) \hat{g}(k)=|a|^{\frac{1}{2}} \overline{\hat{f}(a k)} \hat{g}(k),
$$

where $\hat{f}, \hat{f}_{a}^{*}, \hat{g}$ are the Plancherel transforms of $f, f_{a}^{*}, g$. By applying the Plancherel Theorem for $\mathbb{R}$ we get

$$
\begin{aligned}
\left\|C_{f} g\right\|_{2}^{2} & =\int_{\mathbb{R}_{\neq 0}} \int_{\mathbb{R}}|a|^{-2}\left|\left(f_{a}^{*} * g\right)(b)\right|^{2} \mathrm{~d} a \mathrm{~d} b \\
& =\int_{\mathbb{R}_{\neq 0}} \int_{\mathbb{R}}|a|^{-2}\left|\widehat{f_{a}^{*} * g}(k)\right|^{2} \mathrm{~d} a \mathrm{~d} k \\
& =\int_{\mathbb{R}_{\neq 0}} \int_{\mathbb{R}}|a|^{-1}|\overline{\hat{f}(a k)} \hat{g}(k)|^{2} \mathrm{~d} a \mathrm{~d} k \\
& =\int_{\mathbb{R}_{\neq 0}} \int_{\mathbb{R}}|a k|^{-1}|\overline{\hat{f}(a k)} \hat{g}(k)|^{2}|k| \mathrm{d} a \mathrm{~d} k \\
& =\int_{\mathbb{R}_{\neq 0}} \int_{\mathbb{R}}|l|^{-1}|\overline{\hat{f}(l)} \hat{g}(k)|^{2} \mathrm{~d} l \mathrm{~d} k \\
& =\|g\|_{2}^{2} \cdot \int_{\mathbb{R}_{\neq 0}} \frac{|\hat{f}(l)|^{2}}{|l|} \mathrm{d} l .
\end{aligned}
$$

Since by assumption $C_{f} g$ is in $L^{2}\left(G_{\text {aff }}(\mathbb{R})\right)$, it follows that the function $f$ satisfies

$$
\int_{\mathbb{R}_{\neq 0}} \frac{|\hat{f}(l)|^{2}}{|l|} \mathrm{d} l<\infty
$$

On the other hand, if we assume that $f$ satisfies ineq. (3.3) then we get

$$
\|g\|_{2}^{2} \cdot \int_{\mathbb{R}_{\neq 0}} \frac{|\hat{f}(l)|^{2}}{|l|} \mathrm{d} l=\left\|C_{f} g\right\|_{2}^{2} .
$$


by a calculation analogous to eq. (3.2).

Remark 3.1. Note that the crucial part in either direction is that for $f, g \in L^{2}(\mathbb{R})$ the convolution $f * g$ is in $L^{2}(\mathbb{R})$ if and only if the product $\hat{f} \hat{g}$ is in $L^{2}(\mathbb{R})$, and in that case we have

$$
\widehat{f * g}=\hat{f} \hat{g} .
$$

The proof is not very hard. Since $f * g$ can be considered as a tempered distribution $\left(f * g \in \mathcal{S}^{\prime}(\mathbb{R})\right)$ one can compute the Fourier transform $\mathcal{F}_{\mathcal{S}^{\prime}}(f * g)$ in the distributional sense and show that

$$
\mathcal{F}_{\mathcal{S}^{\prime}}(f * g)=\hat{f} \hat{g}
$$

As the Fourier transform of a Schwartz function coincides with its Plancherel transform and the Schwartz functions form a dense subspace of $L^{2}(\mathbb{R})$ it follows by duality that the distributional Fourier transform $\mathcal{F}_{\mathcal{S}^{\prime}}(u)$ of a function $u \in L^{2}(\mathbb{R})$ coincides with its Plancherel transform $\hat{u}$ (see Lem. B.1). Therefore, $f * g \in L^{2}(\mathbb{R})$ if and only if $\hat{f} \hat{g} \in L^{2}(\mathbb{R})$ and

$$
\widehat{f * g}=\mathcal{F}_{\mathcal{S}^{\prime}}(f * g)
$$

in that case.

In summary, it follows that the (possibly unbounded) operator $V_{f}: L^{2}(\mathbb{R}) \rightarrow L^{2}\left(G_{\text {aff }}(\mathbb{R})\right)$, $V_{f} g=C_{f} g$ is an isometry if and only if $f$ satisfies

$$
\int_{\mathbb{R}_{\neq 0}} \frac{|\hat{f}(l)|^{2}}{|l|} \mathrm{d} l=1
$$

Eq. (3.4) is called Calderón condition and goes back to Calderón [19]. Surely, there exist functions satisfying this condition.

Motivated by this construction, we say that a unitary representation $(\pi, \mathcal{H})$ of a locally group is square-integrable $\rrbracket^{1}$ if there exists a nonzero vector $f \in \mathcal{H}$ such that the coefficient functions $C_{f} g$ are square-integrable for all $g \in \mathcal{H}$, i.e.,

$$
C_{f} g=\langle\pi(\cdot) f, g\rangle \in L^{2}(G) \quad \forall g \in \mathcal{H},
$$

and the operator

$$
V_{f}: \mathcal{H} \rightarrow L^{2}(G), \quad g \mapsto C_{f} g,
$$

is an isometry. In that case $V_{f}$ is called a continuous wavelet transformation and

\footnotetext{
${ }^{1}$ There are different definitions of square-integrable representations. See rem. 3.3 for a discussion on this notion.
} 
$f$ is called an admissible vector (or an admissible function).

\subsubsection{Shearlets and semidirect products}

The classical continuous wavelet transformation comes from the affine group of the real line $G_{\text {aff }}(\mathbb{R})=\mathbb{R} \rtimes \mathbb{R}_{\neq 0}$ which acts on $\mathbb{R}$ by translation and dilation. It generalizes in a straightforward way to the $n$-dimensional similitude group

$$
\operatorname{SIM}(n)=\mathbb{R}^{n} \rtimes\left(\mathbb{R}_{>0} \cdot \mathrm{SO}(n)\right)=\left\{(v, a A) \mid v \in \mathbb{R}^{n}, a \in \mathbb{R}_{>0}, A \in \operatorname{SO}(n)\right\}
$$

acting on $\mathbb{R}^{n}$ by translation, dilation, and rotation. (For details, see Murenzi [54].)

Another way to generalize the classical continuous wavelet transformation are the (generalized) shearlet transformations, which are related to groups of the form $\mathbb{R}^{n} \rtimes H$ with $H=D \cdot S$, where $D$ is a group of the form

$$
D=\left\{ \pm\left(\begin{array}{ccc}
a^{\delta_{1}} & & \\
& \ddots & \\
& & a^{\delta_{n}}
\end{array}\right) \mid 0<a\right\}
$$

with $0<\delta_{n} \leq \cdots \leq \delta_{1} \leq 1$ and $S$ is a closed abelian subgroup of

$$
\left\{\left(\begin{array}{cccc}
1 & s_{1,2} & \cdots & s_{1, n} \\
& 1 & \ddots & \vdots \\
& & \ddots & s_{n-1, n} \\
& & & 1
\end{array}\right) \mid s_{i, j} \in \mathbb{R} \text { for } 1 \leq i<j \leq n\right\} \text {. }
$$

$D$ corresponds to (anisotropic) dilations whereas $S$ corresponds to shearing. (For details, see Alberti et al. [1, Kutyniok and Labate [48]).

Both classes are examples of groups of the form

$$
G=\mathbb{R}^{n} \rtimes H=\left\{(v, A) \mid v \in \mathbb{R}^{n}, A \in H\right\},
$$

where $H$ is a closed subgroup of $\mathrm{Gl}(n)$. $G$ naturally acts on $\mathbb{R}^{n} \cong G / H$ by

$$
(v, A) x=v+A x, \quad \text { for } x \in \mathbb{R}^{n},
$$

which induces a unitary representation $\pi$ of $G$ on $L^{2}\left(\mathbb{R}^{n}\right)$ given by

$$
\pi(v, A) f(x):=|\operatorname{det}(A)|^{-\frac{1}{2}} f\left(A^{-1}(x-v)\right) .
$$

When studying the square-integrability of $\pi$, the approach is similar for all groups of 
the form $G=\mathbb{R}^{n} \rtimes H$. By using the Fourier transformation $L^{2}\left(\mathbb{R}^{n}\right) \rightarrow L^{2}\left(\mathbb{R}^{n}\right), f \mapsto \hat{f}$,

$$
\hat{f}(k):=\int_{\mathbb{R}^{n}} f(x) e^{2 \pi i\langle k, x\rangle} \mathrm{d} x, \quad \text { for } f \in L^{1}\left(\mathbb{R}^{n}\right) \cap L^{2}\left(\mathbb{R}^{n}\right),
$$

the representation $\pi$ can be rewritten as

$$
\hat{\pi}(v, A) \hat{f}(k):=|\operatorname{det}(A)|^{\frac{1}{2}} e^{2 \pi i\langle k, v\rangle} \hat{f}\left(A^{t} k\right)
$$

and the coefficient functions take the form

$$
\begin{aligned}
C_{f} g(v, A): & =\langle\pi(v, A) f, g\rangle=\langle\hat{\pi}(v, A) \hat{f}, \hat{g}\rangle \\
& =\int_{\mathbb{R}^{n}}|\operatorname{det}(A)|^{\frac{1}{2}} e^{-2 \pi i\langle k, v\rangle} \hat{f}\left(A^{t} k\right) \hat{g}(k) \mathrm{d} k .
\end{aligned}
$$

By the Plancherel Theorem the coefficient functions satisfy

$$
\begin{aligned}
\left\|C_{f} g\right\|_{2}^{2} & :=\int_{G} \mathrm{~d}(A, v)|\langle\pi(v, A) f, g\rangle|^{2}=\int_{\mathbb{R}^{n}} \mathrm{~d} v \int_{H} \mathrm{~d} A|\operatorname{det}(A)|^{-1}|\langle\pi(v, A) f, g\rangle|^{2} \\
& =\int_{\mathbb{R}^{n}} \mathrm{~d} k \int_{H} \mathrm{~d} A\left|\hat{f}\left(A^{t} k\right)\right|^{2}|\hat{g}(k)|^{2},
\end{aligned}
$$

in the extended sense that the left-hand side is finite if and only if the right-hand side is and in that case they coincide. In order to get $\left\|C_{f} g\right\|_{2}=\|g\|_{2}$ for all $g \in L^{2}\left(\mathbb{R}^{n}\right), f$ has to satisfy

$$
\int_{H} \mathrm{~d} A\left|\hat{f}\left(A^{t} k\right)\right|^{2} \equiv 1
$$

almost everywhere. A direct consequence is that the stabilizers $H_{k}=\left\{A \in H \mid A^{t} k=\right.$ $k\}$ have to be compact for almost every $k \in \mathbb{R}^{n}$ as the integrand in eq. (3.6) is constant on the cosets modulo $H_{k}$.

For the similitude group and the shearlet groups constructed in [1] almost all stabilizers are indeed compact. Furthermore, in each of the examples there exists a $k_{0} \in \mathbb{R}^{n}$ such that the orbit $\mathcal{O}_{k_{0}}=\left\{A^{t} k_{0} \mid A \in H\right\} \subset \mathbb{R}^{n}$ is open and co-null with respect to the Lebesgue measure on $\mathbb{R}^{n}$. As a consequence, the respective representation $\pi$ is irreducible in both cases (cf. Führ [34, Cor. 21]). Moreover, one can substitute $A k_{0}$ with $A \in H$ for $l \in \mathbb{R}^{n}$ and write

$$
1 \stackrel{!}{=} \int_{H} \mathrm{~d} A\left|\hat{f}\left(A^{t} k_{0}\right)\right|^{2}=\int_{\mathbb{R}^{n}} \mathrm{~d} l \delta(l)|\hat{f}(l)|^{2},
$$

where $\delta$ is the corresponding transformation density (Jacobi determinant) for the substitution $A k_{0} \rightarrow l$. The restriction $\left.\delta\right|_{\mathcal{O}_{k_{0}}}$ is continuous (in fact, it is smooth). By choosing $f \in L^{2}\left(\mathbb{R}^{n}\right)$ such that supp $\hat{f}$ is compact and contained in $\mathcal{O}_{k_{0}}$ we can make sure that $0<\int_{\mathbb{R}^{n}} \mathrm{~d} l \delta(l)|\hat{f}(l)|^{2}<\infty$. Hence, by normalizing $f$ in a suitable way we find a function $f \in L^{2}\left(\mathbb{R}^{n}\right)$ satisfying condition eq. (3.7). Therefore, the representations obtained from the similitude group and the shearlet groups constructed in [1] are 
square-integrable and admit a continuous wavelet transformation.

In the general case of a group of the form $G=\mathbb{R}^{n} \rtimes H$ the situation is more challenging, since the orbit space $\mathbb{R}^{n} / H=\left\{\mathcal{O}_{k} \mid k \in \mathbb{R}^{n}\right\}$ can be much more complicated. A rigorous discussion can be found in Führ [34]. As mentioned above, for square-integrability it is necessary that for almost all $k \in \mathbb{R}^{n}$ (with respect to the Lebesgue measure) the stabilizers $H_{k}=\left\{A \in H \mid A^{t} k=k\right\}$ are compact. Führ shows that another necessary condition is that the orbit space $\mathbb{R}^{n} / H$ is sufficiently regular (see [34] for details). Then there exists a measure $\bar{\nu}$ on $\mathbb{R}^{n} / H$ and a measurable cross-section $\mathbb{R}^{n} / H \rightarrow \mathbb{R}^{n}$, $\mathcal{O} \mapsto k_{\mathcal{O}}$, such that the Lebesgue measure can be decomposed as

$$
\int_{\mathbb{R}^{n}} \varphi(k) \mathrm{d} k=\int_{\mathbb{R}^{n} / H} \int_{H} \varphi\left(A^{t} k_{\mathcal{O}}\right) \frac{1}{\delta\left(A^{t} k_{\mathcal{O}}\right)} \mathrm{d} A \mathrm{~d} \bar{\nu}(\mathcal{O}), \quad \forall \varphi \in L^{1}\left(\mathbb{R}^{n}\right),
$$

for some measurable function $\delta: \mathbb{R}^{n} \rightarrow \mathbb{R}_{\geq 0}$, which is positive almost everywhere and depends on $H, \bar{\nu}$, and the cross-section $\mathcal{O} \mapsto k_{\mathcal{O}}$. In that case, a function $f \in L^{2}\left(\mathbb{R}^{n}\right)$ is admissible if and only if

$$
\int_{H}\left|\hat{f}\left(A^{t} k_{\mathcal{O}}\right)\right|^{2} \mathrm{~d} A=1
$$

for $\bar{\nu}$-almost all $\mathcal{O} \in \mathbb{R}^{n} / H$. Führ shows that for nonunimodular groups a function satisfying eq. (3.8) always exists. For unimodular groups, admissible functions exist if and only if $\bar{\nu}\left(\mathbb{R}^{n} / H\right)<\infty$.

\subsubsection{Windowed Fourier transformation and coherent state transfor- mation}

Another type of generalized continuous wavelet transformations can be constructed from the Heisenberg-Weyl group, which is given by

$$
\begin{aligned}
\operatorname{HW}(n) & =\left\{(q, p, c) \mid q, p \in \mathbb{R}^{n}, c \in \mathbb{R}\right\}, \\
(q, p, c)\left(q^{\prime}, p^{\prime}, c^{\prime}\right) & =\left(q+q^{\prime}, p+p^{\prime}, c+c^{\prime}+\frac{1}{2}\left(\left\langle q, p^{\prime}\right\rangle-\left\langle q^{\prime}, p\right\rangle\right)\right), \\
(q, p, c)^{-1} & =(-q,-p,-c),
\end{aligned}
$$

where $\langle\cdot, \cdot\rangle$ is the standard scalar product on $\mathbb{R}^{n}$. The Heisenberg-Weyl group is a nilpotent Lie group with center

$$
Z=\{(0,0, c) \mid c \in \mathbb{R}\}
$$

and quotient group

$$
\mathrm{HW}(n) / Z \cong\left(\mathbb{R}^{2 n},+\right) .
$$

$\mathrm{HW}(n)$ has two classes of irreducible unitary representations: The 1-dimensional representations of $\mathrm{HW}(n) / Z$ and the infinite-dimensional Schrödinger representations $\pi_{h}$ for $h \in \mathbb{R}_{\neq 0}$, which are induced from nontrivial characters of $Z$. They are defined on 
$L^{2}\left(\mathbb{R}^{n}\right)$ and have the form

$$
\pi_{h}(q, p, c) f(x)=e^{2 \pi i h\left(c+\frac{1}{2}\langle q, p\rangle-\langle x, p\rangle\right)} f(x-q) .
$$

Central elements $(0,0, c)$ act by multiplication with a scalar $e^{2 \pi i h c}$ and, in consequence, the coefficient functions satisfy the relations

$$
\begin{aligned}
C_{f} g(q, p, c) & :=\left\langle\pi_{h}(q, p, c) f, g\right\rangle=e^{-2 \pi i h c}\left\langle\pi_{h}(q, p, 0) f, g\right\rangle, \\
\left|C_{f} g(q, p, c)\right|^{2} & =\left|\left\langle\pi_{h}(q, p, c) f, g\right\rangle\right|^{2}=\left|\left\langle\pi_{h}(q, p, 0) f, g\right\rangle\right|^{2} .
\end{aligned}
$$

Since the modulus of coefficient functions is independent of $c \in Z$ and $Z$ is not compact, the representations $\pi_{h}$ are not square-integrable. However, $(q, p, c) \mapsto\left|C_{f} g(q, p, c)\right|^{2}$ can be considered as a function on $\mathrm{HW}(n) / Z$. Then, using the Fourier transformation and the Plancherel Theorem, it is straightforward to compute

$$
\int_{\mathbb{R}^{n}} \mathrm{~d} q \int_{\mathbb{R}^{n}} \mathrm{~d} p\left|C_{f} g(q, p, c)\right|^{2}=\|f\|_{2}^{2}\|g\|_{2}^{2} .
$$

Hence, for all functions $f \in L^{2}\left(\mathbb{R}^{n}\right)$ with $\|f\|_{2}^{2}=1$, the maps $A_{f}, B_{f}: L^{2}\left(\mathbb{R}^{n}\right) \rightarrow$ $L^{2}\left(\mathbb{R}^{2 n}\right)$,

$$
\begin{gathered}
A_{f} g(q, p)=\left\langle\pi_{h}\left(q, p,-\frac{1}{2}\langle q, p\rangle\right) f, g\right\rangle=\int_{\mathbb{R}^{n}} \mathrm{~d} x e^{2 \pi i h\langle x, p\rangle} \overline{f(x-q)} g(x), \\
B_{f} g(q, p)=\left\langle\pi_{h}(q, p, 0) f, g\right\rangle=\int_{\mathbb{R}^{n}} \mathrm{~d} x e^{2 \pi i h\langle x, p\rangle} \overline{f\left(x-\frac{1}{2} q\right)} g\left(x+\frac{1}{2} q\right),
\end{gathered}
$$

are isometries.

$A_{f}$ is called windowed or short-time Fourier transform. Its discrete version plays an important role in signal and image processing (see for instance the book [11] by Blanchet and Charbit). The idea is due to Gabor [36] who considered the case $n=1$ and used a Gaussian function $f(x)=\left(\frac{2 a}{\pi}\right)^{\frac{1}{4}} e^{2 \pi i(k x+c)} e^{-a x^{2}}$.

The map $B_{f}$ has applications in quantum mechanics. Here, $f$ is usually chosen to be a Gaussian function,

$$
f(x)=\left(\frac{2^{n} \operatorname{det} C}{\pi^{n}}\right)^{\frac{1}{4}} e^{2 \pi i\langle x, k\rangle} e^{-\langle x, C x\rangle},
$$

as well. Those functions, which play an important role in quantum mechanics, are called coherent states (cf. [3]) and the map $B_{f}$ is also called coherent state transformation.

The construction presented for the Heisenberg-Weyl group can be generalized to other locally compact groups $G$. By Schur's Lemma it is clear that for any irreducible unitary representation $\pi$ central elements $z \in Z(G)$ are mapped to multiplication operators $\pi(z)=e^{2 \pi i \alpha(z)} \mathbb{1}_{\pi}$. Hence, the modulus of a coefficient function $|\langle\pi(x) f, g\rangle|$ is constant on cosets modulo $Z(G)$. In order for $\pi$ to be square-integrable, it is necessary that $Z(G)$ is compact. However, as $Z(G)$ is a normal subgroup of $G$ it seems natural to consider $|\langle\pi(x) f, g\rangle|$ as a function on $G / Z(G)$, instead, and say that $f$ is admissible 
(modulo $Z(G)$ ) if

$$
\int_{G / Z(G)}|\langle\pi(\sigma(y)) f, g\rangle|^{2} \mathrm{~d} y=\|g\|_{\pi}^{2}, \quad \forall g \in \mathcal{H}_{\pi},
$$

for some Borel section $\sigma: G / Z(G) \rightarrow G$. In that case, $\pi$ is called square-integrable modulo $Z(G)$.

In [55], Perelomov goes even a step further. For a given $f_{0} \in \mathcal{H}_{\pi}$ let

$$
H=\left\{x \in G \mid \exists \alpha(x) \in \mathbb{R}: \pi(x) f_{0}=e^{2 \pi i \alpha(x)} f_{0}\right\} .
$$

$H$ is the maximal subgroup of $G$ such that $|\langle\pi(\cdot) f, g\rangle|$ is constant on cosets modulo $H$ for all $g \in \mathcal{H}_{\pi}$. If there exists a $G$-invariant measure $\nu$ on $X=G / H$ then an irreducible representation $\pi$ is said to be square-integrable modulo $H$ if

$$
\int_{G / H}\left|\left\langle\pi(\sigma(y)) f_{0}, g\right\rangle\right|^{2} \mathrm{~d} \nu(y)=\|g\|_{\pi}^{2}, \quad \forall g \in \mathcal{H}_{\pi} .
$$

By the irreducibility of $\pi$ it is sufficient to check that

$$
\int_{G / H}\left|\left\langle\pi(\sigma(y)) f_{0}, f_{0}\right\rangle\right|^{2} \mathrm{~d} \nu(y)=\left\|f_{0}\right\|_{\pi}^{2} .
$$

In [55, Perelomov studies the existence of admissible vectors in this generalized sense for different classes of groups, like nilpotent Lie groups and semisimple Lie groups, and gives sufficient and necessary conditions for many examples.

\subsubsection{Coherent states over coadjoint orbits}

The notation square-integrability modulo a subgroup led to more generalizations. Principally, one could take any subgroup $H<G$ and a suitable Borel section $\sigma: G / H \rightarrow G$ and ask for the existence of an element $f \in \mathcal{H}$ such that

$$
\int_{G / H}|\langle\pi(\sigma(y)) f, g\rangle|^{2} \mathrm{~d} \nu_{\sigma}(y)=\|g\|_{\mathcal{H}}^{2}, \quad \forall g \in \mathcal{H} .
$$

Certainly, in many cases $|\langle\pi(\sigma(y)) f, g\rangle|$ will not be constant on cosets modulo $H$ and, therefore, the existence of an $f$ satisfying condition (3.9) depends on the choice of $\sigma$. If such an $f$ exists then $\pi$ is called square-integrable modulo $(H, \sigma)$ (cf. Ali, Antoine, and Gazeau [3]).

It turns out that finding suitable $H$ and $\sigma$ for a given representation $\pi$ is a hard task. One approach comes from geometric quantization or, to be more precise, from Kirillov's orbit method. The basic idea of the orbit method is to associate irreducible unitary representations of a Lie group with its coadjoint orbits (cf. Kirillov [45], 46], Auslander and Kostant [8]). If for example $G$ is a nilpotent, simply connected, connected Lie group then there is a one-to-one correspondence between the coadjoint orbits $\mathcal{O} \in \mathfrak{g}^{*} / \operatorname{Ad}^{*}(G)$ 
and the irreducible unitary representations $\pi \in \hat{G}$ of $G$. Suppose that the orbit $\mathcal{O} \cong$ $G / H$ is an affine subspace of $\mathfrak{g}^{*}$. Then the representation $\left(\pi_{\mathcal{O}}, \mathcal{H}_{\mathcal{O}}\right)$ corresponding to $\mathcal{O}$ is square-integrable modulo $H$ (cf. Moscovici [53, Thm. 3.1]). Moreover, there exists a continuous homomorphism $\alpha: H \rightarrow \mathbb{R}$ such that

$$
\pi_{\mathcal{O}}(h) f=e^{2 \pi i \alpha(h)} f
$$

for all $h \in H$ and $f \in \mathcal{H}_{\mathcal{O}}$ (cf. Perelomov [55, Chp. 10]). Indeed, the Heisenberg-Weyl group HW $(n)$ discussed in Chp. 3.1 .3 is an example for this.

Unfortunately, in many cases there is no $f \in \mathcal{H}$ satisfying the assumptions of Perelomov's construction. Nevertheless, it is possible to find a cross-section $\sigma: G / H \cong \mathcal{O} \rightarrow G$ such that $\pi_{\mathcal{O}}$ is square-integrable modulo $(H, \sigma)$. For example, in [20] de Bièvre studies irreducible representations of semidirect products $G=\mathbb{R}^{n} \rtimes H$ with $H \leq \operatorname{Gl}(n)$. Given an irreducible representation $\pi$, the author constructs such a cross-section and shows that under certain conditions $\pi$ is square-integrable modulo $(H, \sigma)$. De Bièvre states that the construction works for several groups, for example, for the Euclidean group $\mathbb{R}^{n} \rtimes \mathrm{SO}(n)$, the Galilei group and the Poincaré group in $1+1$ dimensions.

\subsubsection{Continuous wavelet transformations on manifolds}

In [5] and [6], Antoine and Vandergheynst discuss the generalization of the usual continuous wavelet transformation coming from the similitude group (see Chp. 3.1.2) to manifolds like the $n$-sphere or the two-sheet hyperboloid.

They consider a manifold $M$ together with a group which acts transitively on $M$ and contains some kind of local dilations. Motivated by Holschneider [40] they want to construct a continuous wavelet transformation which - in terms of group contractions (cf. 2.4 - reduces locally to the usual continuous wavelet transformation on $\mathbb{R}^{n}$.

In [6] Antoine and Vandergheynst, present an explicit construction for the 2-sphere. As its symmetry group $\mathrm{SO}(3)$ does not contain any local dilations they extend it to the conformal group and consider $\mathrm{SO}_{0}(3,1)$, the connected component of the identity of $\mathrm{SO}(3,1)$. The easiest way to describe the conformal transformations is to represent the 2-sphere as a subset of the 3 -dimensional projective space $\mathrm{P}\left(\mathbb{R}^{4}\right)$

$$
S^{2}=\left\{\mathbb{R}\left(\begin{array}{c}
x_{1} \\
x_{2} \\
x_{3} \\
r
\end{array}\right) \in \mathrm{P}\left(\mathbb{R}^{4}\right) \mid x_{1}^{2}+x_{2}^{2}+x_{3}^{2}=r^{2}\right\} .
$$


It is the image of the set $\mathbb{S}^{2}=\left\{x \in \mathbb{R}^{3} \mid\|x\|^{2}=1\right\}$ under the embedding $\mathbb{R}^{3} \rightarrow \mathrm{P}\left(\mathbb{R}^{4}\right)$,

$$
\left(\begin{array}{c}
x_{1} \\
x_{2} \\
x_{3}
\end{array}\right) \mapsto \mathbb{R}\left(\begin{array}{c}
x_{1} \\
x_{2} \\
x_{3} \\
1
\end{array}\right)
$$

The group $G=\mathrm{PSO}(3,1) \cong \mathrm{SO}_{0}(3,1)$ acts on $S^{2}$ by matrix-vector multiplication.

Locally the group $\mathrm{SO}_{0}(3,1) \cong \operatorname{PSO}(3,1)$ acts on $S^{2}$ by translation, rotations and dilation, which can be described as follows. There are two different geometric pictures we want to use.

- One is stereographic projection. Let $s=\mathbb{R}\left(\begin{array}{c}0 \\ 0 \\ -1 \\ 1\end{array}\right)$ and write the analogue of the stereographic projection $\Phi: S^{2} \backslash\{s\} \rightarrow \mathbb{R}^{2}$ by

$$
\Phi: \mathbb{R}\left(\begin{array}{c}
x_{1} \\
x_{2} \\
x_{3} \\
r
\end{array}\right) \mapsto \frac{2}{x_{3}+r}\left(\begin{array}{c}
x_{1} \\
x_{2}
\end{array}\right), \quad \Phi^{-1}:\left(\begin{array}{c}
y_{1} \\
y_{2}
\end{array}\right) \mapsto \mathbb{R}\left(\begin{array}{c}
4 y_{1} \\
4 y_{2} \\
4-\left(y_{1}^{2}+y_{2}^{2}\right) \\
4+\left(y_{1}^{2}+y_{2}^{2}\right)
\end{array}\right) .
$$

Then it is straightforward to verify that elements $D_{a} \in \operatorname{PSO}(3,1)$ of the form

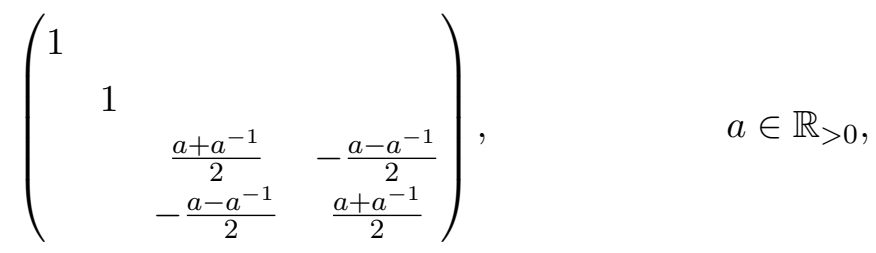

act on $\mathbb{R}^{2}$ by

$$
\Phi \circ D_{a} \circ \Phi^{-1}:\left(\begin{array}{l}
y_{1} \\
y_{2}
\end{array}\right) \mapsto a\left(\begin{array}{l}
y_{1} \\
y_{2}
\end{array}\right)
$$

The stereographic projection can be interpreted as a projection of a neighborhood of $s$ onto the tangent space of $S^{2}$ at $s$. In that picture the $D_{a} \in \operatorname{PSO}(3,1)$ can be interpreted as dilations on a small neighborhood of $s$.

- The other one is group contraction (see Chp.2.4. The basic idea is to increase the radius $R$ of the sphere and watch how the group action in a small neighborhood of some point $p \in S^{2}$ changes as $R$ goes to infinity. Speaking in terms of Def. 2.78 we are describing the contraction of $G=\operatorname{PSO}(3,1)$ along the stabilizer $H$ of $p$, where the parameter $r=\frac{1}{R}$ goes to 0 . The subgroup $H$ is isomorphic to

$$
H \cong \mathbb{R}^{2} \rtimes\left(\mathbb{R}_{>0} \cdot \mathrm{SO}(2)\right)=N \rtimes\left(\mathbb{R}_{>0} \cdot \mathrm{SO}(2)\right)
$$


and the contracted group $G_{0}$ is isomorphic to

$$
G_{0} \cong \mathbb{R}^{2} \rtimes\left(\mathbb{R}^{2} \rtimes\left(\mathbb{R}_{>0} \cdot \mathrm{SO}(2)\right)\right)=V \rtimes\left(N \rtimes\left(\mathbb{R}_{>0} \cdot \mathrm{SO}(2)\right)\right)
$$

$H$ acts on $V=\mathbb{R}^{2}$ by

$$
(x, a A) v=a A v, \quad x \in N, a \in \mathbb{R}_{>0}, A \in \mathrm{SO}(2), v \in V .
$$

Therefore, the elements $(v, x, a A)$ of the group $G_{0}=V \rtimes H$ act on $V$ by translation by $v$, rotation by $A$, and dilation by $a$.

The representation Antoine and Vandergheynst study in [6] is the quasi-regular representation $\pi$ on $L^{2}\left(S^{2}\right)$. It is given by

$$
\pi(A) f(\omega)=\lambda(A, \omega)^{\frac{1}{2}} f\left(A^{-1} \omega\right), \quad \text { for } f \in L^{2}\left(S^{2}\right), A \in \operatorname{PSO}(3,1), \omega \in S^{2},
$$

where

$$
\lambda(A, \omega):=\frac{\mathrm{d}\left(A^{-1} \omega\right)}{\mathrm{d} \omega}
$$

is the Radon-Nikodym derivative with respect to the $\mathrm{SO}(3)$-invariant measure on $\mathbb{S}^{2}$. $\pi$ is an irreducible, unitary representation of $\operatorname{PSO}(3,1)$, which is not square-integrable.

Antoine and Vandergheynst show that $\pi$ contracts to the representation $\pi_{0}$ of $G_{0}$ on $L^{2}(V)$ given by

$$
\pi_{0}(v, x, a A) f(w)=a^{-1} f\left(a^{-1} A^{-1}(w-v)\right), \quad(v, x, a A) \in V \rtimes\left(N \rtimes\left(\mathbb{R}_{>0} \mathrm{SO}(2)\right)\right) .
$$

The kernel of $\pi_{0}$ is $N \unlhd G_{0}$ and $G_{0} / N$ is isomorphic to $\operatorname{SIM}(2)$. As shown in Chp. 3.1.2 $\pi_{0}$ is square-integrable with respect to the group $\operatorname{SIM}(2)$ and in that sense it is squareintegrable modulo $N$ (with respect to $G_{0}$ ). Therefore, it seems plausible to investigate whether $\pi$ is square-integrable modulo $(N, \sigma)$ for some Borel section $\sigma: G / N \rightarrow G$. The image of the section $\sigma$ chosen in [6] is $X=\operatorname{SO}(3) \times\left\{D_{a} \mid a \in \mathbb{R}_{>0}\right\}$, which contains the motions $\mathrm{SO}(3)$ on $\mathbb{S}^{2}$ (embedded in $\mathrm{PSO}(3,1)$ via eq. 3.10 ) and the dilations $D_{a}$ defined by eq. (3.11). Antoine and Vandergheynst conjecture that there is still no function which is admissible in the sense defined in Chp. 3.1.4 and say that a function $f \in L^{2}\left(S^{2}\right)$ is a continuous frame modulo $(N, \sigma)$ if there exist constants $0<m<M<\infty$ such that

$$
m\|g\|_{2}^{2} \leq \int_{\mathrm{SO}(3)} \mathrm{d} A \int_{\mathbb{R}_{>0}} \frac{\mathrm{d} a}{a^{3}}\left|\left\langle\pi\left(A D_{a}\right) f, g\right\rangle\right|^{2} \leq M\|g\|_{2}^{2},
$$

where $\mathrm{d} A \frac{\mathrm{d} a}{a} \frac{1}{a^{2}}$ is the pushforward (with respect to $\sigma$ ) of the $G$-invariant measure on 
$G / N$. For a continuous frame $f$ the map $V_{f}: L^{2}\left(S^{2}\right) \rightarrow L^{2}(X)$,

$$
V_{f}(A, a)=\left\langle\pi\left(A D_{a}\right) f, g\right\rangle,
$$

is bounded and $V_{f}^{*} V_{f}: L^{2}\left(S^{2}\right) \rightarrow L^{2}\left(S^{2}\right)$ has a bounded inverse. However, it is an isometry only if $m$ and $M$ can be chosen to be equal. In general, $T_{f}:=V_{f}^{*} V_{f}$ will not be (a multiple of) the identity of $L^{2}\left(S^{2}\right)$ and, therefore, the reconstruction formula has to be corrected by $T_{f}^{-1} V_{f}^{*}: L^{2}(X) \rightarrow L^{2}\left(S^{2}\right)$,

$$
F \mapsto \int_{\mathrm{SO}(3)} \mathrm{d} A \int_{\mathbb{R}_{>0}} \frac{\mathrm{d} a}{a^{3}} F(A, a) T_{f}^{-1} \pi\left(A D_{a}\right) f .
$$

In the construction of Antoine and Vandergheynst, the operator $T_{f}$ turns out to be $\mathrm{SO}(3)$-invariant. Hence, the continuous wavelet transformation constructed in [6] respects the $\mathrm{SO}(3)$-symmetry of the sphere.

\subsubsection{Continuous diffusion wavelet transformations}

The definition of a suitable set of dilations is desirable for applications and seems necessary for the existence of a generalized continuous wavelet transformation. In contrast to the geometric construction discussed in Chp. 3.1.5there is another approach coming from physics or partial differential equations. In [26, Ebert and Wirth construct a wavelet transformation on (homogeneous spaces of) a compact group $G$ by solving the diffusion (or heat) equation

$$
\Delta_{G} u(t, a)=\partial_{t} u(t, a), \quad t \in \mathbb{R}_{>0}, a \in G,
$$

where $\Delta_{G}$ is the Laplace-Beltrami operator. For a given initial condition $u(0, a)=u_{0}(a)$ the solution of the diffusion equation is

$$
u(t, a)=\left(u_{0} * k_{t}\right)(a), \quad \text { for } t>0,
$$

where $k_{t}$ is the diffusion (or heat) kernel, which can be computed explicitly (cf. [26]). Moreover, $k_{t}$ is an approximate convolution identity, meaning that for all $\varphi \in L^{p}(G)$, $1 \leq p<\infty$,

$$
\varphi * k_{t} \underset{t \rightarrow 0}{\longrightarrow} \varphi
$$

in the topology of $L^{p}(G)$. Ebert and Wirth construct a family $\Psi=\left(\psi_{t}\right)_{t \in \mathbb{R}>0} \subseteq L^{2}(G)$ such that

$$
\psi_{t}^{*} * \psi_{t}=-\partial_{t} k_{t}
$$


where $\psi_{t}^{*}(a)=\overline{\psi_{t}\left(a^{-1}\right)}$. Thus, $\Psi$ satisfies

$$
\int_{t}^{\infty} \psi_{s}^{*} * \psi_{s} \mathrm{~d} s=k_{t}-\lim _{s \rightarrow \infty} k_{t}=k_{t}-k_{\infty}
$$

$k_{\infty}$ is the (nonzero) constant function satisfying $\int_{G} k_{\infty}(a) \mathrm{d} a=1$. In conclusion for all $f \in L^{2}(G)$ with zero-mean $\left(\left\langle k_{\infty}, f\right\rangle=0\right)$ one can verify that

$$
\int_{0}^{\infty} \int_{G}\left|C_{\psi_{t}} f(a)\right|^{2} \mathrm{~d} a \mathrm{~d} t=\int_{0}^{\infty}\left\langle f * \psi_{t}^{*} * \psi_{t}, f\right\rangle \mathrm{d} t=\|f\|_{2}^{2} .
$$

Hence, the map $W_{\Psi}: L_{0}^{2}(G) \rightarrow L^{2}\left(\mathbb{R}_{>0} \times G\right)$,

$$
W_{\Psi} f(t, a)=C_{\psi_{t}} f(a)
$$

where $L_{0}^{2}(G)=\left\{f \in L^{2}(G) \mid \int_{G} f(a) \mathrm{d} a=0\right\}$, defines an isometry.

For small $t$ the functions $k_{t}$ and $\psi_{t}$ are localized at $e \in G$. When $t$ goes to infinity $k_{t}$ and $\psi_{t}$ melt down and distribute uniformly on $G$. In that sense changing the parameter $t \in \mathbb{R}_{>0}$ describes some kind of dilation.

The key element of the construction of Ebert and Wirth is that the solution of the diffusion equation can be written in terms of a convolution kernel which is in $L^{1}(G)$. The reason for this is among others that all irreducible representations are finite dimensional. In [35], Führ discusses a similar construction for simply connected, connected nilpotent Lie groups $N$. As nilpotent Lie groups, in general, have infinite dimensional irreducible representations, the solution of the diffusion equation is given by convolution with a distribution rather than a function. Therefore, Führ considers the equation $\partial_{t} u=Q u$ with initial condition $u(0, \cdot)=u_{0}$, where $Q$ is a negative definite self-adjoint operator $Q$ on $L^{2}(N)$ which is left-invariant. Under suitable assumptions its solution is given by convolution with a function $k_{t}$ which is contained in the domain of $Q$. Führ shows that the family $\left(\psi_{t}\right)_{t \in \mathbb{R}_{>0}}=\left(2 t\left(Q k_{t}\right)^{*}\right)_{t \in \mathbb{R}_{>0}}$ is admissible in the sense that

$$
\int_{\mathbb{R}_{>0}} \frac{\mathrm{d} t}{t}\left\|C_{\psi_{t}} f\right\|_{L^{2}(N)}^{2}=\|f\|_{L^{2}(N)}^{2}
$$

where

$$
C_{k_{t}} f(x)=\left\langle\lambda_{N}(x) \psi_{t}, f\right\rangle=2 t\left(f * Q k_{t}\right)(x)
$$

and $\lambda_{N}$ is the left regular representation of $N$.

\subsection{Group-theoretical approach to continuous wavelet transformations}

In the following, we will see a summary of the most important results on continuous wavelet transformations coming from a systematic, group-theoretical analysis. It is an 
excerpt from the textbook [33] by Führ and all facts presented here can be found therein (if not otherwise stated). To get a better understanding, we will see not only the results but also their proofs or, at least, a sketch of them. It this way, we can highlight the technical problems and obstructions and how to solve them. For a rigorous survey and detailed proofs see [33].

\subsubsection{Introduction and definitions}

Let $G$ be a locally compact group and let $(\pi, \mathcal{H})$ be a unitary representation. For vectors $f, g \in \mathcal{H}$, let $C_{f} g \in C_{b}(G)$ be the coefficient function

$$
C_{f} g: a \mapsto\langle\pi(a) f, g\rangle, \quad a \in G \text {. }
$$

To be more precise, $C_{f} g$ is a coefficient function of the contragredient representation $\pi$ or the complex conjugate of a coefficient function of $\pi$. For a fixed $\psi \in \mathcal{H}$, it defines an operator $C_{\psi}: \mathcal{H} \rightarrow C_{b}(G)$ from $\mathcal{H}$ to the continuous bounded functions on $G$. We are particularly interested in the case where $C_{\psi} f \in L^{2}(G)$.

Definition 3.2. Let $G$ be a locally compact group and $(\pi, \mathcal{H})$ a unitary representation. Let $\psi \in \mathcal{H}$ be a fixed, nonzero vector.

(a) Denote by $V_{\psi}$ the possibly unbounded (not even densely defined) operator $\mathcal{H} \rightarrow$ $L^{2}(G)$ given by $V_{\psi} f=C_{\psi} f$ on the domain

$$
\operatorname{dom}\left(V_{\psi}\right)=\left\{f \in \mathcal{H} \mid C_{\psi} f \in L^{2}(G)\right\} .
$$

(b) If $V_{\psi}$ is an isometry, then it is called a continuous wavelet transformation. In that case $\psi$ is called an admissible vector.

(c) If a given representation $\pi$ possesses an admissible vector then $\pi$ is called squareintegrable.

Remark 3.3. There are different definitions of square-integrable representations. Some authors say that a unitary representation $(\pi, \mathcal{H})$ is square-integrable if there exists a nonzero vector $f \in \mathcal{H}$ such that $C_{f} f \in L^{2}(G)$ (cf. Wong [59] for instance). In earlier articles $\pi$ is said to be square-integrable if $\pi$ is irreducible and there exists $f \in \mathcal{H} \backslash\{0\}$ such that $C_{f} f \in L^{2}(G)$ (cf. Mackey [52]). The latter are nowadays referred to as discrete series representations.

For irreducible representations all these definitions coincide. Indeed, if $\pi$ is irreducible and square-integrable (as in Def. 3.2) and $f \in \mathcal{H}$ is an admissible vector then

$$
\left\|C_{f} f\right\|_{2}=\left\|V_{f} f\right\|_{2}=\|f\|_{\mathcal{H}}<\infty .
$$

The converse is less obvious and can be found in Wong [59, Thm. 6.1]. 
Given an admissible vector $\psi$, the continuous wavelet transformation $V_{\psi}$ is an isometry. Its adjoint $V_{\psi}^{*}: L^{2}(G) \rightarrow \mathcal{H}$ can be written as

$$
V_{\psi}^{*} F=\int_{G} \pi(a) \psi \cdot F(a) \mathrm{d} a,
$$

which converges in the weak sense. The operator $V_{\psi}^{*} V_{\psi}$ is the identity on $\mathcal{H}$ and for all $f \in \mathcal{H}$ we get the reconstruction formula

$$
f=\int_{G} \pi(a) \psi \cdot\langle\pi(a) \psi, f\rangle \mathrm{d} a .
$$

Conversely, the operator $V_{\psi} V_{\psi}^{*}$ is the orthogonal projection from $L^{2}(G)$ onto the image of $V_{\psi}$. It is an integral operator given by

$$
\left[V_{\psi} V_{\psi}^{*} F\right](a)=\int_{G}\langle\pi(a) \psi, \pi(b) \psi\rangle F(b) \mathrm{d} b, \quad \forall F \in L^{2}(G) .
$$

A direct consequence of Def. 3.2 is the following proposition.

Proposition 3.4. Let $(\pi, \mathcal{H})$ be a square-integrable representation of $G$. Then $\pi$ is a subrepresentation of the left regular representation $\lambda_{G}$.

Proof. Let $\psi \in \mathcal{H}$ be admissible. Then, for all $f \in \mathcal{H}$ and $a, b \in G$,

$$
V_{\psi}(\pi(a) f)(b)=\left\langle\pi\left(a^{-1} b\right) \psi, f\right\rangle=V_{\psi} f\left(a^{-1} b\right)=\left[\lambda_{G}(a) V_{\psi} f\right](b) .
$$

Hence, $V_{\psi}$ is an isometric intertwiner. This, $\pi$ is a subrepresentation of $\lambda_{G}$.

The importance of Prop. 3.4 lies in the fact that we only have to study squareintegrability for subrepresentations of the left regular representation. In particular, we can use the Plancherel decomposition for this purpose.

Another result which follows immediately is that subrepresentations of square-integrable representations are square-integrable.

Proposition 3.5. Let $\left(\pi, \mathcal{H}_{\pi}\right)$ be a square-integrable representation of $G$ and let $\left(\rho, \mathcal{H}_{\rho}\right)$ be a subrepresentation of $\pi$. Then $\rho$ is square-integrable.

Proof. Let $S: \mathcal{H}_{\rho} \rightarrow \mathcal{H}_{\pi}$ be an isometric intertwiner and let $\psi \in \mathcal{H}_{\pi}$ be admissible for $\pi$. Then $S^{*} \psi$ is admissible for $\rho$, as

$$
\begin{aligned}
C_{S^{*} \psi}^{\rho} f(a) & =\left\langle\rho(a) S^{*} \psi, f\right\rangle_{\rho}=\left\langle S^{*} \pi(a) \psi, f\right\rangle_{\rho}=\langle\pi(a) \psi, S f\rangle_{\pi}=V_{\psi}^{\pi} S f(a), \\
\left\|C_{S^{*} \psi}^{\rho} f\right\|_{\rho}^{2} & =\left\|V_{\psi}^{\pi} S f\right\|_{\pi}^{2}=\|S f\|_{\pi}^{2}=\|f\|_{\rho}^{2},
\end{aligned}
$$

for all $f \in \mathcal{H}_{\rho}$.

One may ask whether all subrepresentations of the left regular representation are square-integrable. By Prop. 3.5 it would be sufficient to show that the regular representation is square-integrable. For type I nonunimodular groups this is actually the 
case as we will see in Thm. 3.15. For unimodular groups, however, the left regular representation is not square-integrable in most cases.

Theorem 3.6. Suppose that $G$ is unimodular. Then its left regular representation $\lambda_{G}$ is square-integrable if and only if $G$ is discrete.

Proof (sketch). If $G$ is discrete, then the Haar measure of $\left\{e_{G}\right\}$ is positive. One can directly verify that $\psi \in L^{2}(G)$, defined by

$$
\psi(a)=\left\{\begin{array}{ll}
\left|\left\{e_{G}\right\}\right|^{-1}, & a=e_{G} \\
0, & a \neq e_{G}
\end{array},\right.
$$

is admissible. This is due to $V_{\psi}=\operatorname{id}_{L^{2}(G)}$ in that case.

If $\lambda_{G}$ is square-integrable and $\psi \in L^{2}(G)$ is admissible, then by the reconstruction formula every $f \in L^{2}(G)$ can be written as

$$
f=\int_{G} \lambda_{G}(a) \psi \cdot V_{\psi} f(a) \mathrm{d} a=V_{\psi} f * \psi
$$

As the convolution of two $L^{2}$-functions is continuous, it follows that $L^{2}(G) \subseteq C_{b}(G)$. Therefore, $\left\{e_{G}\right\}$ cannot be a null set. Thus, $G$ is discrete.

A detailed proof, which provides more information on the last step, can be found in Führ [33, Thm. 2.42].

\subsubsection{Square-integrability condition}

Similarly to the simple example of the group $G_{\text {aff }}(\mathbb{R})$ discussed in Chp. 3.1.1, the (generalized) Fourier transformation is the key to the admissibility condition. However, since the general setup is more complicated, we have to work harder than in the simple case. The first step is to carefully define the Fourier transform of a coefficient function of the left regular representation. As before, it will turn out to take a particularly easy form. Then, we can investigate under which conditions the Fourier transform is square-integrable. In that case, the Plancherel Theorem makes sure that the coefficient function is square-integrable, as well. Finally, we study under which assumptions a given representation has admissible vectors.

From now on suppose that $G$ is a second countable locally compact group and denote its left regular representation by $\lambda_{G}$. If $\lambda_{G}$ is type I then the Plancherel measure of $G$ is denoted by $\mu_{G}$ (cf. Thm. 2.67 and Thm. 2.69) and the operators defined in Lem. 2.68 are denoted by $\left\{D_{\sigma}\right\}_{\sigma \in \hat{G}}$. As discussed in Chp. 2.3.5. Führ states the Plancherel Theorem for groups $G$ of type I having the property that the kernel of the modular function $N=\operatorname{ker} \Delta_{G}$ is of type I and is regularly embedded in $G$. Hence, in Führ [33] those conditions appear in the results which are based on the Plancherel decomposition. However, since the Plancherel Theorem only requires that $\lambda_{G}$ is type I, the requirements of the results taken from [33] can be relaxed. 
Let $f, g \in L^{2}(G)$ and let $C_{f} g \in C_{0}(G), C_{f} g(a)=\left\langle f, \lambda_{G}(a) g\right\rangle$, be a coefficient function. In general, $C_{f} g$ will neither be in $L^{1}(G)$ nor in $L^{2}(G)$. Let $\mathcal{A}(G)$ be the set of all such coefficient functions. The space $\mathcal{A}(G)$ actually has more structure than one might expect.

Theorem 3.7. Let $G$ be a second countable locally compact group and let

$$
\mathcal{A}(G)=\left\{C_{f} g \mid f, g \in L^{2}(G)\right\}
$$

and endow $\mathcal{A}(G)$ with the norm

$$
\|t\|_{\mathcal{A}}=\inf \left\{\|f\|_{2}\|g\|_{2} \mid f, g \in L^{2}(G), t=C_{f} g\right\} .
$$

$\mathcal{A}(G)$ is closed under pointwise addition, multiplication, and conjugation. Together with the norm $\|\cdot\|_{\mathcal{A}}$, it is a Banach ${ }^{*}$-algebra.

The algebra $\mathcal{A}(G)$ is called the Fourier algebra of $G$. It was originally invented to study abelian locally compact groups. In [28], Eymard generalized it to nonabelian locally compact groups and studied their properties. Thm. 3.7 follows from [28, Thm. p. 218].

The Fourier algebra can also be defined differently, namely, as the image of the inverse Fourier transform. Recall that $\mathcal{B}_{1}^{\oplus}(\hat{G}) \subseteq \mathcal{B}^{\oplus}(\hat{G})$ is the space of direct integral operators $\int_{\hat{G}}^{\oplus} T(\sigma) \mathrm{d} \mu_{G}(\sigma)$ satisfying

$$
\|T\|_{1}=\int_{\hat{G}}\|T(\sigma)\|_{\sigma, 1} \mathrm{~d} \mu_{G}(\sigma)<\infty,
$$

where $\|\cdot\|_{\sigma, 1}$ is the trace-norm of $\mathcal{B}_{1}\left(\mathcal{H}_{\sigma}\right)$. Define $\mathcal{F}_{\mathcal{A}}^{-1}: \mathcal{B}_{1}^{\oplus}(\hat{G}) \rightarrow C_{0}(G)$ by

$$
\mathcal{F}_{\mathcal{A}}^{-1}(T)(a)=\int_{\hat{G}} \operatorname{Tr}_{\sigma}\left[\sigma(a)^{*} T(\sigma)\right] \mathrm{d} \mu_{G}(\sigma) .
$$

In [33], Führ shows that $\mathcal{F}_{\mathcal{A}}$ has the following properties.

Theorem 3.8. Let $G$ be a second countable locally compact group and suppose that $\lambda_{G}$ is type I. Then $\mathcal{F}_{\mathcal{A}}^{-1}(T) \in \mathcal{A}(G)$ for all $T \in \mathcal{B}_{1}^{\oplus}(\hat{G})$. Moreover, $\mathcal{F}_{\mathcal{A}}^{-1}: \mathcal{B}_{1}^{\oplus}(\hat{G}) \rightarrow \mathcal{A}(G)$ is an isometric isomorphism of Banach spaces.

Proof. Let $T=\{T(\sigma)\}_{\sigma \in \hat{G}} \in \mathcal{B}_{1}^{\oplus}(\hat{G})$ and denote $t=\mathcal{F}_{\mathcal{A}}^{-1}(T)$. Let

$$
T(\sigma)=U(\sigma)|T(\sigma)|
$$

be the pointwise polar decomposition of $T$ and let

$$
\begin{aligned}
U & =\{U(\sigma)\}_{\sigma \in \hat{G}} \in \mathcal{B}(\hat{G}), & |T| & =\{|T(\sigma)|\}_{\sigma \in \hat{G}} \in \mathcal{B}_{1}^{\oplus}(\hat{G}) \\
S_{1} & =\left\{U(\sigma)|T(\sigma)|^{\frac{1}{2}}\right\}_{\sigma \in \hat{G}} \in \mathcal{B}_{2}^{\oplus}(\hat{G}), & S_{2} & =\left\{|T(\sigma)|^{\frac{1}{2}}\right\}_{\sigma \in \hat{G}} \in \mathcal{B}_{2}^{\oplus}(\hat{G}) .
\end{aligned}
$$


By Lem. A.1 the fields of operators $U,|T|, S_{1}$, and $S_{2}$ are measurable. If we denote the inverse Plancherel transforms of $S_{1}$ and $S_{2}$ by $s_{1}=\mathcal{P}^{-1}\left(S_{1}\right), s_{2}=\mathcal{P}^{-1}\left(S_{2}\right)$, then

$$
\begin{aligned}
t(a) & =\int_{\hat{G}} \operatorname{Tr}_{\sigma}\left[T(\sigma) \sigma(a)^{*}\right] \mathrm{d} \mu_{G}(\sigma) \\
& =\int_{\hat{G}} \operatorname{Tr}_{\sigma}\left[S_{1}(\sigma) S_{2}(\sigma)^{*} \sigma(a)^{*}\right] \mathrm{d} \mu_{G}(\sigma) \\
& =\left\langle\lambda_{G}(a) s_{2}, s_{1}\right\rangle \\
& =C_{s_{2}} s_{1}(a)
\end{aligned}
$$

Hence, $\|t\|_{\mathcal{A}} \leq\left\|s_{1}\right\|_{2}\left\|s_{2}\right\|_{2}=\|T\|_{1}$. Therefore, $\mathcal{F}_{\mathcal{A}}^{-1}$ is bounded and $\left\|\mathcal{F}_{\mathcal{A}}^{-1}(T)\right\|_{\mathcal{A}} \leq\|T\|_{1}$, for all $T \in \mathcal{B}_{1}^{\oplus}(\hat{G})$.

Let $t \in \mathcal{A}(G)$. For a given $\epsilon>0$, let $f, g \in L^{2}(G)$ such that $t=C_{f} g$ and

$$
\|t\|_{\mathcal{A}}+\epsilon \geq\|f\|_{2}\|g\|_{2}
$$

Let $T=\left\{\hat{g}(\sigma) \hat{f}(\sigma)^{*}\right\}_{\sigma \in \hat{G}}$ and note that $t=\mathcal{F}_{\mathcal{A}}^{-1}(T)$ as

$$
\mathcal{F}_{\mathcal{A}}^{-1}(T)=\int_{\hat{G}} \operatorname{Tr}_{\sigma}\left[\hat{g}(\sigma) \hat{f}(\sigma)^{*} \sigma(a)^{*}\right] \mathrm{d} \mu_{G}(\sigma)=\left\langle\lambda_{G}(a) f, g\right\rangle=t(a) .
$$

$T$ satisfies the estimate

$$
\begin{aligned}
\|T\|_{1} & =\int_{\hat{G}} \operatorname{Tr}_{\sigma}\left[\left|\hat{g}(\sigma) \hat{f}(\sigma)^{*}\right|\right] \mathrm{d} \mu_{G}(\sigma) \\
& \leq \int_{\hat{G}}\|\hat{g}(\sigma)\|_{2}\left\|\hat{f}(\sigma)^{*}\right\|_{2} \mathrm{~d} \mu_{G}(\sigma) \\
& \leq\|g\|_{2}\|f\|_{2} \\
& \leq\|t\|_{\mathcal{A}}+\epsilon .
\end{aligned}
$$

The first inequality follows from

$$
\operatorname{Tr}[|A B|]=\operatorname{Tr}\left[U^{*} A B\right] \leq\left\|U^{*} A\right\|_{2}\|B\|_{2}=\|A\|_{2}\|B\|_{2},
$$

where $A, B$ are Hilbert-Schmidt operators and $A B=U|A B|$ is the polar decomposition of $A B$.

Hence, $\mathcal{F}_{\mathcal{A}}^{-1}$ is surjective and $\left\|\mathcal{F}_{\mathcal{A}}^{-1}(T)\right\|_{\mathcal{A}} \geq\|T\|_{1}$, for all $T \in \mathcal{B}_{1}^{\oplus}(\hat{G})$.

Thm. 3.8 shows that any coefficient function $C_{f} g$ can be Fourier transformed using $\mathcal{F}_{\mathcal{A}}$.

Corollary 3.9. Let $\mathcal{F}_{\mathcal{A}}: \mathcal{A} \rightarrow \mathcal{B}_{1}^{\oplus}(\hat{G})$ be the inverse of $\mathcal{F}_{\mathcal{A}}^{-1}$. Then,

$$
\mathcal{F}_{\mathcal{A}}\left(C_{f} g\right)=\hat{g} \hat{f}^{*} \in \mathcal{B}_{1}^{\oplus}(\hat{G})
$$

for all $f, g \in L^{2}(G)$.

The following theorem is one of the most important results in [33. It provides a tool 
to check whether a coefficient function is square-integrable.

Theorem 3.10. Let $T=\{T(\sigma)\}_{\sigma \in \hat{G}} \in \mathcal{B}_{1}^{\oplus}(\hat{G})$ and define

$$
t(a)=\int_{\hat{G}} \operatorname{Tr}_{\sigma}\left[T(\sigma) \sigma(a)^{*}\right] \mathrm{d} \mu_{G}(\sigma) .
$$

Then $t \in L^{2}(G)$ if and only if $\left\{T(\sigma) D_{\sigma}^{-1}\right\}_{\sigma \in \hat{G}} \in \mathcal{B}_{2}^{\oplus}(\hat{G})$. In that case, $\hat{t}(\sigma)=T(\sigma) D_{\sigma}^{-1}$ $\mu_{G}$-almost everywhere.

Proof (sketch). The first direction is rather easy to prove. For simplicity, denote $\left\{T(\sigma) D_{\sigma}^{-1}\right\}_{\sigma \in \hat{G}}$ by $S=\{S(\sigma)\}_{\sigma \in \hat{G}}$. If $S \in \mathcal{B}_{2}^{\oplus}(\hat{G})$ then $\mathcal{P}^{-1}(S)=s \in L^{2}(G)$. For all $\varphi \in C_{c}(G)$ we have $\int_{G} \overline{\varphi(a)}(t(a)-s(a)) \mathrm{d} a=0$ because of

$$
\begin{aligned}
\int_{G} \overline{\varphi(a)} t(a) \mathrm{d} a & =\int_{\hat{G}} \operatorname{Tr}_{\sigma}\left[T(\sigma) \sigma(\varphi)^{*}\right] \mathrm{d} \mu_{G}(\sigma) \\
& =\int_{\hat{G}} \operatorname{Tr}_{\sigma}\left[T(\sigma) D_{\sigma}^{-1} D_{\sigma} \sigma(\varphi)^{*}\right] \mathrm{d} \mu_{G}(\sigma) \\
& =\int_{\hat{G}} \operatorname{Tr}_{\sigma}\left[S(\sigma) \hat{\varphi}(\sigma)^{*}\right] \mathrm{d} \mu_{G}(\sigma) \\
& =\int_{G} \overline{\varphi(a)} s(a) \mathrm{d} a .
\end{aligned}
$$

Hence, $s=t$ almost everywhere. In particular, $t \in L^{2}(G)$ and $\hat{t}=T(\sigma) D_{\sigma}^{-1} \mu_{G}$-almost everywhere.

The converse direction is much more involving and very technical. A complete proof of the theorem can be found in Führ [33, Thm. 4.15].

Thm. 3.10 is the key to the following result.

Corollary 3.11. For $f, g \in L^{2}(G)$ we have $C_{f} g \in L^{2}(G)$ if and only if

$$
\left\{\hat{g}(\sigma) \hat{f}(\sigma)^{*} D_{\sigma}^{-1}\right\}_{\sigma \in \hat{G}} \in \mathcal{B}_{2}^{\oplus}(\hat{G}) .
$$

In that case, its Plancherel transform has the form

$$
\widehat{C_{f}} g(\sigma)=\hat{g}(\sigma) \hat{f}(\sigma)^{*} D_{\sigma}^{-1}
$$

almost everywhere.

As described above, we defined the Fourier transform of a coefficient function and showed that it has the easy form $\mathcal{F}_{\mathcal{A}}\left(C_{f} g\right)=\hat{g} \hat{f}^{*}$ for $f, g \in L^{2}(G)$. Moreover, we know that $C_{f} g$ is square-integrable if and only if $\left[\sigma \mapsto \hat{g}(\sigma) \hat{f}(\sigma)^{*} D_{\sigma}^{-1}\right]$ is. With this knowledge, we can study whether a subrepresentation $\pi$ of the left regular representation $\lambda_{G}$ has admissible vectors. The following proposition helps to understand how $\pi$ is embedded in $\lambda_{G}$. 
Proposition 3.12. If $(\pi, \mathcal{H})$ is a subrepresentation of $\lambda_{G}$ then there exists a measurable field of orthogonal projections $\{P(\sigma)\}_{\sigma \in \hat{G}}$ such that

$$
P: L^{2}(G) \rightarrow \mathcal{H}
$$

is the orthogonal projection onto $\mathcal{H}$.

Proof. This result is a direct consequence of Thm. 2.71. Since the orthogonal projection $P: L^{2}(G) \rightarrow \mathcal{H}$ intertwines the left regular representation $\lambda_{G}, P$ is contained in $\mathcal{C}\left(\lambda_{G}\right)$. Therefore, there is a measurable field of operators $\{P(\sigma)\}_{\sigma \in \hat{G}}$ such that $P=\int_{\hat{G}}^{\oplus} P(\sigma) \mathrm{d} \mu_{G}(\sigma)$. Since $P$ satisfies $P=P^{2}=P^{*}$, it follow that

$$
P(\sigma)=P(\sigma)^{2}=P(\sigma)^{*}
$$

almost everywhere.

With help of Prop. 3.12 we can characterize admissible functions as follows.

Theorem 3.13. Let $(\pi, \mathcal{H})$ be a subrepresentation of $\lambda_{G}$ and let $P: L^{2}(G) \rightarrow \mathcal{H}$ be the orthogonal projection onto the closed subspace $\mathcal{H} \subseteq L^{2}(G)$. Then the following are equivalent.

(i) $f \in \mathcal{H}$ is admissible.

(ii) $V_{f} V_{f}^{*}=P$.

(iii) $\hat{f}(\sigma)^{*} D_{\sigma}^{-2} \hat{f}(\sigma)=P(\sigma)$ almost everywhere.

Proof. The equivalence $(i) \Leftrightarrow(i i)$ follows from the definition of admissibility.

If $f$ is admissible and $V_{f} V_{f}^{*}=P$, then (iii) follows from Prop. 3.12. If (iii) is fulfilled then $V_{f}$ is bounded by Cor. 3.11 and, in particular,

$$
\left\|V_{f} g\right\|_{2}^{2}=\int_{\hat{G}}\left\|\hat{g}(\sigma) \hat{f}(\sigma)^{*} D_{\sigma}^{-1}\right\|_{2}^{2} \mathrm{~d} \mu_{G}(\sigma)=\int_{\hat{G}}\|\hat{g}(\sigma)\|_{2}^{2} \mathrm{~d} \mu_{G}(\sigma)=\|g\|_{2}^{2},
$$

implying that $f$ is admissible. This shows that $(i) \wedge(i i) \Rightarrow(i i i) \Rightarrow(i)$.

In a straightforward way, Thm. 3.13 can be used to characterize square-integrability of representations of unimodular groups.

Theorem 3.14. Suppose that $G$ is unimodular. Then a subrepresentation $\pi \leq \lambda_{G}$ is square-integrable if and only if

$$
c=\int_{\hat{G}} m_{\pi}(\sigma) \mathrm{d} \mu_{G}(\sigma)<\infty,
$$

where $m_{\pi}(\sigma)=\operatorname{rk} P(\sigma)$. In that case any admissible vector $f$ has norm $\|f\|_{\pi}^{2}=c$. 
Proof. Suppose $\pi$ is square-integrable and $f$ is admissible. Then

$$
\begin{aligned}
\|f\|_{\pi}^{2} & =\int_{\hat{G}} \operatorname{Tr}_{\sigma}\left[\hat{f}(\sigma)^{*} \hat{f}(\sigma)\right] \mathrm{d} \mu_{G}(\sigma) \\
& =\int_{\hat{G}} \operatorname{Tr}_{\sigma}[P(\sigma)] \mathrm{d} \mu_{G}(\sigma) \\
& =\int_{\hat{G}} m_{\pi}(\sigma) \mathrm{d} \mu_{G}(\sigma)
\end{aligned}
$$

In particular $\int_{\hat{G}} m_{\pi}(\sigma) \mathrm{d} \mu_{G}(\sigma)<\infty$.

Now suppose that inequality 3.12 is fulfilled. Then

$$
\int_{\hat{G}}\|P(\sigma)\|_{2}^{2} \mathrm{~d} \mu_{G}(\sigma)=\int_{\hat{G}} \operatorname{Tr}_{\sigma}[P(\sigma)] \mathrm{d} \mu_{G}(\sigma)=\int_{\hat{G}} m_{\pi}(\sigma) \mathrm{d} \mu_{G}(\sigma)<\infty .
$$

Hence $P \in \mathcal{B}_{2}^{\oplus}(\hat{G})$ and $p=\mathcal{P}^{-1}(P) \in \mathcal{H}$ as $P p=p$. Moreover, $V_{p}$ is an isometry as $V_{p} g=g$ for all $g \in \mathcal{H}$. Therefore, $p$ is admissible and $\pi$ is square-integrable.

For nonunimodular groups the situation is more complicated. Because of the presence of the operators $\left\{D_{\sigma}\right\}_{\sigma \in \hat{G}}$, we have to be more careful.

Theorem 3.15. Suppose that $G$ is nonunimodular. Then $\lambda_{G}$ is square-integrable.

Proof (sketch). By Thm. 3.13 (iii) we have to show that there is a measurable field of operators $\hat{f} \in \mathcal{B}_{2}^{\oplus}(\hat{G})$ such that $D_{\sigma}^{-1} \hat{f}(\sigma)$ is an isometry almost everywhere. Let $\left(e_{n}\right)_{n \in \mathbb{N}}$ be an orthonormal measurable structure for $\int_{\hat{G}}^{\oplus} \mathcal{H}_{\sigma} \mathrm{d} \mu_{\hat{G}}(\sigma)$ (cf. Chp. 2.3.2). Suppose we can find a set of measurable vector fields $\left(v_{n}\right)_{n \in \mathbb{N}}$ such that $\left(D_{\sigma}^{-1} v_{n}(\sigma)\right)$ are orthonormal in $\mathcal{H}_{\sigma}$ for almost all $\sigma \in \hat{G}$ and $\sum_{n \in \mathbb{N}}\left\|v_{n}\right\|^{2}<\infty$. Then $\hat{f}$ given by

$$
\hat{f}(\sigma)=\sum_{n \in \mathbb{N}} v_{n}(\sigma) \otimes e_{n}(\sigma)^{*}
$$

has the desired properties as

$$
\begin{aligned}
\hat{f}(\sigma)^{*} D_{\sigma}^{-2} \hat{f}(\sigma) & =\sum_{m \in \mathbb{N}} \sum_{n \in \mathbb{N}}\left\langle D_{\sigma}^{-1} v_{m}(\sigma), D_{\sigma}^{-1} v_{n}\right\rangle e_{m}(\sigma) \otimes e_{n}(\sigma)^{*} \\
& =\sum_{n \in \mathbb{N}} e_{n}(\sigma) \otimes e_{n}(\sigma)^{*} \\
& =\operatorname{id}_{\mathcal{H}_{\sigma}}
\end{aligned}
$$

almost everywhere, and

$$
\begin{aligned}
\|\hat{f}\|_{2}^{2} & =\int_{\hat{G}} \sum_{m \in \mathbb{N}} \sum_{n \in \mathbb{N}}\left\langle v_{m}(\sigma), v_{n}(\sigma)\right\rangle\left\langle e_{n}(\sigma), e_{m}(\sigma)\right\rangle \mathrm{d} \mu_{G}(\sigma) \\
& =\int_{\hat{G}} \sum_{n \in \mathbb{N}}\left\langle v_{n}(\sigma), v_{n}(\sigma)\right\rangle \mathrm{d} \mu_{G}(\sigma) \\
& =\sum_{n \in \mathbb{N}}\left\|v_{n}\right\|^{2} .
\end{aligned}
$$


The construction of the family $\left(v_{n}\right)_{n \in \mathbb{N}}$ is quite technical and is based on an explicit description of the operators $\left(D_{\sigma}\right)_{\sigma \in \hat{G}}$. The detailed proof can be found in Führ [33, Thm. 4.23]. To get an idea, suppose that $u=\{u(\sigma)\}_{\sigma \in \hat{G}}$ is a measurable field of vectors with $\|u(\sigma)\|=1$ and $u(\sigma) \in \operatorname{dom}\left(D_{\sigma}\right)$ almost everywhere. Furthermore, suppose that $\sigma \mapsto\left\|D_{\sigma} u(\sigma)\right\|^{2}$ is locally integrable, i.e.,

$$
\int_{\hat{G}} \operatorname{char}_{E}(\sigma) \cdot\left\|D_{\sigma} u(\sigma)\right\|^{2} \mathrm{~d} \mu_{G}(\sigma)<\infty
$$

for all measurable $E \subseteq \hat{G}$ of finite Plancherel measure, where $\operatorname{char}_{E}$ is the characteristic function of $E$. For example, $u$ can be a field of vectors coming from the measurable structure of $\int_{\hat{G}}^{\oplus} \mathcal{H}_{\sigma} \mathrm{d} \mu_{G}(\sigma)$. In [33], $u$ is chosen such that $\sigma \mapsto\left\|D_{\sigma} u(\sigma)\right\|^{2}$ is a bounded function.

By using the following trick we can construct a measurable field of vectors $v=\{v(\sigma)\}_{\sigma \in \hat{G}}$ from $u=\{u(\sigma)\}_{\sigma \in \hat{G}}$ satisfying

$$
\int_{\hat{G}}\|v(\sigma)\|^{2} \mathrm{~d} \mu_{G}(\sigma)<\infty \quad \text { and } \quad\left\|D_{\sigma}^{-1} v(\sigma)\right\|^{2}=1
$$

almost everywhere. Since $\hat{G}$ is $\sigma$-finite, there exists a pairwise disjoint family $\left\{E_{n}\right\}_{n \in \mathbb{N}}$ of measurable subsets of $\hat{G}$ having finite Plancherel measure and satisfying $\hat{G}=\bigcup_{n \in \mathbb{N}} E_{n}$. As im $\Delta_{G}$ is a nontrivial subgroup of $\mathbb{R}_{>0}$ and gets arbitrarily close to zero, we can find elements $a_{n} \in G, n \in \mathbb{N}$ such that

$$
\int_{\hat{G}} \operatorname{char}_{E_{n}}(\sigma) \cdot\left\|D_{\sigma} u(\sigma)\right\|^{2} \mathrm{~d} \mu_{G}(\sigma) \leq \frac{1}{2^{n} \Delta_{G}\left(a_{n}\right)} .
$$

Define $v=\{v(\sigma)\}_{\sigma \in \hat{G}}$ by

$$
v(\sigma)=D_{\sigma} \sigma\left(a_{n}\right) u(\sigma)=\Delta_{G}^{1 / 2}\left(a_{n}\right) \sigma\left(a_{n}\right) D_{\sigma} u(\sigma) .
$$

Then, $\left\|D_{\sigma}^{-1} v(\sigma)\right\|=\left\|\sigma\left(a_{n}\right) u(\sigma)\right\|=1$ and

$$
\begin{aligned}
\int_{\hat{G}}\|v(\sigma)\|^{2} \mathrm{~d} \mu_{G}(\sigma) & =\sum_{n \in \mathbb{N}} \int_{\hat{G}} \operatorname{char}_{E_{n}}(\sigma) \cdot \Delta_{G}\left(a_{n}\right) \cdot\left\|D_{\sigma} u(\sigma)\right\|^{2} \mathrm{~d} \mu_{G}(\sigma) \\
& \leq \sum_{n \in \mathbb{N}} 2^{-n} \leq 1
\end{aligned}
$$

With some more effort one can obtain a family $\left(v_{n}\right)_{n \in \mathbb{N}}$ having the properties described above.

Note that the reason why unimodular and nonunimodular groups behave so differently comes from the fact that the subgroup $\operatorname{im}\left(\Delta_{G}\right) \leq \mathbb{R}_{>0}$, and therefore almost all of the operators $D_{\sigma}$, is unbounded for nonunimodular groups. That is why we can find measurable fields of operators $\{\hat{f}(\sigma)\}_{\sigma \in \hat{G}}$ such that $D_{\sigma}^{-1} \hat{f}(\sigma)$ is an isometry and $\{\hat{f}(\sigma)\}_{\sigma \in \hat{G}} \in \mathcal{B}_{2}^{\oplus}(\hat{G})$. For unimodular group, it is, in general, not possible to find 
$\{\hat{f}(\sigma)\}_{\sigma \in \hat{G}}$ such that $\hat{f}(\sigma)$ is an isometry and $\{\hat{f}(\sigma)\}_{\sigma \in \hat{G}} \in \mathcal{B}_{2}^{\oplus}(\hat{G})$.

In summary we get the following final result.

Theorem 3.16. Let $G$ be a second countable locally compact group and suppose that its left regular representation $\lambda_{G}$ is type $I$. Let $(\pi, \mathcal{H})$ be a unitary representation of $G$ of type I and let

$$
\pi \cong \int_{\hat{G}}^{\oplus} m_{\pi}(\sigma) \cdot \sigma \mathrm{d} \mu_{\pi}(\sigma)
$$

be its central decomposition.

If $G$ is unimodular, then $\pi$ is square-integrable if and only if

(i) $\mu_{\pi}$ is absolutely continuous with respect to the Plancherel measurable $\mu_{G}$ of $G$,

(ii) $m_{\pi}(\sigma) \leq \operatorname{dim}(\sigma)$ for $\mu_{G}$-almost all $\sigma \in \hat{G}$,

(iii) and

$$
\int_{\hat{G}} m_{\pi}(\sigma) \mathrm{d} \mu_{G}(\sigma)<\infty
$$

If $G$ is nonunimodular, then $\pi$ is square-integrable if and only if

(i) $\mu_{\pi}$ is absolutely continuous with respect to the Plancherel measurable $\mu_{G}$ of $G$.

Proof. By Thm. 2.66, condition (i) and (ii) are satisfied if and only if $\pi$ is a subrepresentation of $\lambda_{G}$. Since almost all irreducible representations of nonunimodular groups are infinite-dimensional, condition (ii) is automatically fulfilled for those groups.

Then, the first part of the theorem is Thm. 3.14. The second part follows from Thm. 3.15 and Prop. 3.5 . 


\section{CHAPTER 4}

\section{New approaches to continuous wavelet transformations}

This chapter is the main part of this thesis. Here, we develop new strategies to continuous wavelet transformations. In Chp. 4.1, we will see that every representation can be decomposed into a representation which is easy to handle and one which is hard to handle. "Easy to handle" means that it is closely related to the left regular representation and can be studied using the methods presented in Chp. 3.2. In Chp. 4.2 , it is shown that it is always possible to find a generalized continuous wavelet transformation for those representations. For representations which turn out to be hard to handle, more effort is needed to develop generalized continuous wavelet transformations. In Chp. 4.3 . we will see a construction for induced representations of semidirect products and group extensions of a Lie group by the real vector space $\mathbb{R}^{n}$. In the last section, in Chp. 4.4 . the approach by Antoine and Vandergheynst presented in [5] and [6] is reviewed. The authors want to construct a generalized continuous wavelet transformation on manifolds which is closely related to the classical continuous wavelet transformation and its multidimensional generalization (cf. Chp. 3.1.1 and Chp. 3.1.2). We will see that the assumptions made in those articles are too restrictive. Antoine and Vandergheynst use the quasi left regular representation of the $n$-sphere to describe their construction and we will see that the $n$-sphere is in fact the only example satisfying their assumptions. Hence, it is necessary to use strategies which are not related to the classical continuous wavelet transformation and its multidimensional generalization.

Throughout, $G$ will be a second countable locally compact group and its left regular representation will be denoted as $\lambda_{G}$. If $\lambda_{G}$ is type I, then its Plancherel transformation is written as

$$
\mathcal{P}: L^{2}(G) \rightarrow \mathcal{B}_{2}^{\oplus}(\hat{G})=\int_{\hat{G}}^{\oplus} \mathcal{B}_{2}\left(\mathcal{H}_{\sigma}\right) \mathrm{d} \mu_{G}(\sigma), f \mapsto \hat{f} .
$$

The Plancherel measure is denoted by $\mu_{G}$. The Fourier transformation has the form

$$
\begin{gathered}
\mathcal{F}: L^{1}(G) \rightarrow \mathcal{B}^{\oplus}(\hat{G})=\int_{\hat{G}}^{\oplus} \mathcal{B}\left(\mathcal{H}_{\sigma}\right) \mathrm{d} \mu_{G}(\sigma), \\
\mathcal{F}(f)(\sigma)=\sigma(f)=\int_{G} f(a) \cdot \sigma(a) \mathrm{d} a .
\end{gathered}
$$


On $\mathbb{R}^{n}$, we use the convention

$$
\mathcal{P}(f)(k)=\mathcal{F}(f)(k)=\int_{\mathbb{R}^{n}} f(a) \cdot e^{2 \pi i\langle k, a\rangle} \mathrm{d} a, \quad \forall f \in L^{1}\left(\mathbb{R}^{n}\right) \cap L^{2}\left(\mathbb{R}^{n}\right)
$$

for the Plancherel and Fourier transformation.

\subsection{Relation between representations}

Let $G$ be a second countable locally compact group and let $(\pi, \mathcal{H})$ be a representation of $G$. In Chp. 3.2 we saw that a necessary condition for being square-integrable is that $\pi$ is a subrepresentation of $\lambda_{G}$. In this section we will see that any representation $\pi$ can be split into a direct sum $\pi=\pi_{1} \oplus \pi_{2}$ such that $\pi_{1}$ is quasi-equivalent to a subrepresentation of $\lambda_{G}$ and $\pi_{2}$ is disjoint. If $\pi$ is cyclic, then $\pi_{1}$ is a subrepresentation of $\lambda_{G}$.

Recall that the central decomposition of a representation $(\pi, \mathcal{H})$ is a unitary equivalence between $(\pi, \mathcal{H})$ and

$$
\left(\int_{\check{G}}^{\oplus} \pi_{p} \mathrm{~d} \mu(p), \int_{\breve{G}}^{\oplus} \mathcal{H}_{p} \mathrm{~d} \mu(p)\right)
$$

where $\left\{\left(\pi_{p}, \mathcal{H}_{p}\right)\right\}_{p \in \check{G}}$ is a measurable field of representations and $\left(\pi_{p}, \mathcal{H}_{p}\right) \in p$ for $\mu$ almost all $p \in \check{G}$. If $\pi$ is type I, then the central decomposition can be written as

$$
\left(\int_{\hat{G}}^{\oplus} m_{\pi}(p) \cdot \sigma_{p} \mathrm{~d} \mu(p), \int_{\hat{G}}^{\oplus} m_{\pi}(p) \cdot \mathcal{H}_{\sigma_{p}} \mathrm{~d} \mu(p)\right)
$$

where $\left(\sigma_{p}, \mathcal{H}_{\sigma_{p}}\right) \in p$ is a fixed irreducible representation of all $p \in \hat{G}$ and $m_{\pi}$ is the multiplicity function.

The basic idea to prove the statement above is to use the Lebesgue decomposition theorem. It states that, for any two $\sigma$-finite measures $\nu$ and $\nu^{\prime}$, there exists a decomposition $\nu=\nu_{1}+\nu_{2}$ such that $\nu_{1}$ is absolutely continuous with respect to $\nu^{\prime}$, denoted by $\nu_{1} \ll \nu^{\prime}$, and $\nu_{2}$ and $\nu^{\prime}$ are mutually singular, denoted by $\nu_{2} \perp \nu^{\prime}$. (See, for instance, Elstrodt [27. Thm. 2.6].) First, we have to show that the central measure $\mu$ and the Plancherel measure $\mu_{G}$ are $\sigma$-finite.

Lemma 4.1. Let $G$ be a second countable locally compact group and $\pi$ a unitary representation on a separable Hilbert space $\mathcal{H}$. Let

$$
\pi \cong \int_{\check{G}}^{\oplus} \pi_{p} \mathrm{~d} \mu(p)
$$

be a central decomposition of $\pi$. Then $\mu$ is $\sigma$-finite.

Proof. By Kallenberg [43, p. 21], it is sufficient to show that there exists a function $f \in L^{1}(\check{G}, \mu)$ which is positive almost everywhere.

W.l.o.g. we assume that $\pi=\int_{\breve{G}}^{\oplus} \pi_{p} \mathrm{~d} \mu(p)$ and $\mathcal{H}=\int_{\breve{G}}^{\oplus} \mathcal{H}_{p} \mathrm{~d} \mu(p)$. 
As $\mathcal{H}$ is separable, there exists a countable complete orthonormal system $\left(\psi_{n} \mid n \in \mathbb{N}\right)$ of $\mathcal{H}$. Define $f: \check{G} \rightarrow \mathbb{R}_{\geq 0}$ by

$$
f(p)=\sum_{n \in \mathbb{N}} 2^{-n}\left\|\psi_{n}(p)\right\|_{p}^{2} .
$$

Then $f$ is in $L^{1}(\check{G}, \mu)$ as

$$
\int_{\breve{G}} f(p) \mathrm{d} \mu(p)=\int_{\check{G}} \sum_{n \in \mathbb{N}} 2^{-n}\left\|\psi_{n}(p)\right\|_{p}^{2} \mathrm{~d} \mu(p)=\sum_{n \in \mathbb{N}} 2^{-n} \underbrace{\int_{\breve{G}}\left\|\psi_{n}(p)\right\|_{p}^{2} \mathrm{~d} \mu(p)}_{=1}=1 .
$$

We have to verify that $f$ is positive $\mu$-almost everywhere. Therefore, let $B \subset \check{G}$ be a measurable subset such that $\left.f\right|_{B}=0$. Let $P_{B}$ be the orthogonal projection given by

$$
P_{B}=\int_{\check{G}}^{\oplus} \operatorname{char}_{B}(p) \mathbb{1}_{p} \mathrm{~d} \mu(p),
$$

where $\operatorname{char}_{B}$ is the characteristic function of $B$. From the definition of $f$, it follows that

$$
\begin{aligned}
0 & =\int_{\breve{G}} \operatorname{char}_{B}(p) f(p) \mathrm{d} \mu(p)=\int_{\breve{G}} \operatorname{char}_{B}(p) \sum_{n \in \mathbb{N}} 2^{-n}\left\|\psi_{n}(p)\right\|_{p}^{2} \mathrm{~d} \mu(p) \\
& =\sum_{n \in \mathbb{N}} 2^{-n} \int_{\breve{G}} \operatorname{char}_{B}(p)\left\|\psi_{n}(p)\right\|_{p}^{2} \mathrm{~d} \mu(p)=\sum_{n \in \mathbb{N}} 2^{-n} \int_{\breve{G}}\left\|\operatorname{char}_{B}(p) \psi_{n}(p)\right\|_{p}^{2} \mathrm{~d} \mu(p) \\
& =\sum_{n \in \mathbb{N}} 2^{-n}\left\|P_{B} \psi_{n}\right\|^{2}
\end{aligned}
$$

and, therefore, $P_{B} \psi_{n}=0$ for all $n \in \mathbb{N}$. As $\left\{\psi_{n} \mid n \in \mathbb{N}\right\}$ is total in $\mathcal{H}$ it follows that $P_{B}=0$ and, hence, $B$ is a null set.

Now, the Lebesgue decomposition applied to $\mu$ and $\mu_{G}$ yields the following results.

Theorem 4.2. Let $G$ be a second countable locally compact group. Let $(\pi, \mathcal{H})$ be a representation. Then,

- $\pi$ is quasi-equivalent to a subrepresentation of $\lambda_{G}$,

- or $\pi$ and $\lambda_{G}$ are disjoint,

- or there exists a decomposition $\pi=\pi_{1} \oplus \pi_{2}$ such that $\pi_{1}$ is quasi-equivalent to a subrepresentation of $\lambda_{G}$ and $\pi_{2}$ and $\lambda_{G}$ are disjoint.

Proof. Let

$$
\pi \cong \int_{\breve{G}}^{\oplus} \pi_{p} \mathrm{~d} \mu(p)
$$

be the central decomposition of $\pi$ and let $\mu_{G}$ be the Plancherel measure of $G$. Let $\mu=\mu_{1}+\mu_{2}$ be the Lebesgue decomposition of $\mu$ with respect to $\mu_{G}$, i.e., $\mu_{1} \ll \mu_{G}$ and $\mu_{2} \perp \mu_{G}$. Since $\mu_{2}$ and $\mu_{G}$ are mutually singular, there exists a measurable subset 
$E \subseteq \check{G}$ such that $\mu_{2}(E)=0$ and $\mu_{G}(\check{G} \backslash E)=0$. Moreover, $\mu_{1}(\check{G} \backslash E)=0$ as $\mu_{1}$ is absolutely continuous with respect to $\mu_{G}$.

If $\mu_{1} \neq 0$ and $\mu_{2} \neq 0$, let

$$
\begin{aligned}
& \pi_{1}=\int_{\breve{G}}^{\oplus} \pi_{p} \mathrm{~d} \mu_{1}(p), \\
& \pi_{2}=\int_{\breve{G}}^{\oplus} \pi_{p} \mathrm{~d} \mu_{2}(p) .
\end{aligned}
$$

Then $\pi$ is equivalent to $\pi_{1} \oplus \pi_{2}$. By Thm. 2.65, $\pi_{1}$ is quasi-equivalent to a subrepresentation of $\lambda_{G}$, and $\pi_{2}$ and $\lambda_{G}$ are disjoint.

If $\mu_{1}=0$ or $\mu_{2}=0$, then it follows analogously that $\pi$ and $\lambda_{G}$ are disjoint or $\pi \leq \lambda_{G}$, respectively.

Corollary 4.3. Let $G$ be a second countable locally compact group and suppose that its left regular representation $\lambda_{G}$ is type $I$. Let $(\pi, \mathcal{H})$ be a cyclic representation. Then,

- $\pi$ is a subrepresentation of $\lambda_{G}$,

- or $\pi$ and $\lambda_{G}$ are disjoint,

- or there exists a decomposition $\pi=\pi_{1} \oplus \pi_{2}$ such that $\pi_{1}$ is a subrepresentation of $\lambda_{G}$ and $\pi_{2}$ and $\lambda_{G}$ are disjoint.

Proof. It is sufficient to show that a cyclic representation $\pi$ which is quasi-equivalent to a subrepresentation of $\lambda_{G}$ is a subrepresentation of $\lambda_{G}$.

Again, let

$$
\pi \cong \int_{\check{G}}^{\oplus} \pi_{p} \mathrm{~d} \mu(p)
$$

be the central decomposition of $\pi$ and let $\mu_{G}$ be the Plancherel measure of $G$.

Since $\lambda_{G}$ is type I, $\check{G} \backslash \hat{G}$ is $\mu_{G}$-null and thus $\mu$-null. In particular, $\pi$ is type I. Therefore, $\pi$ is equivalent to

$$
\pi \cong \int_{\hat{G}}^{\oplus} m_{\pi}(p) \cdot \sigma_{p} \mathrm{~d} \mu(p)
$$

Let $\psi=\{\psi(p)\}_{p \in \check{p}}$ be a cyclic vector in $\int_{\hat{G}}^{\oplus} m_{\pi}(p) \cdot \mathcal{H}_{\sigma_{p}} \mathrm{~d} \mu(\sigma)$. Then, almost everywhere, the operator

$$
\psi(p) \in m_{\pi}(p) \cdot \mathcal{H}_{\sigma_{p}}=\mathcal{H}_{\sigma_{p}} \otimes \mathcal{H}_{m_{\pi}(p)} \subseteq \mathcal{B}\left(\overline{\mathcal{H}_{m_{\pi}(p)}}, \mathcal{H}_{\sigma_{p}}\right)
$$

has full row rank, i.e., $\operatorname{rk}(\psi(p))=m_{\pi}(p)$. Because of $\operatorname{rk}(\psi(p)) \leq \operatorname{dim}\left(\mathcal{H}_{\sigma_{p}}\right)$ it follows that $m_{\pi}(p) \leq \operatorname{dim}\left(\mathcal{H}_{\sigma_{p}}\right)$ almost everywhere. By Thm. 2.66, $\pi$ is a subrepresentation of $\lambda_{G}$. 
The subrepresentation $\pi_{1} \leq \pi$ is the "good" part of $\pi$ as it is a subrepresentation of $\lambda_{G}$. By Thm. 3.16, $\pi_{1}$ is square-integrable if and only if

- $G$ is nonunimodular,

- or $G$ is unimodular and

$$
\int_{\hat{G}} m_{\pi_{1}}\left(\sigma_{p}\right) \mathrm{d} \mu_{G}(p)<\infty .
$$

The representation $\pi_{2} \leq \pi$ is the "bad" part of $\pi$ as it is never square-integrable. By Thm. 4.2 "good" representations, i.e., subrepresentations of $\lambda_{G}$, and "bad" representations, i.e., those which are disjoint to $\lambda_{G}$, can be studied separately.

In this thesis the main focus is on representations induced from an irreducible representation of a subgroup. Let $H \leq G$ be a closed subgroup of $G$ and let $\chi$ be a representation of $H$. Let $\pi=\operatorname{ind}_{H}^{G}(\chi)$.

Theorem 4.4. If $\chi$ is a cyclic representation of $H$, then $\pi=\operatorname{ind}_{H}^{G}(\chi)$ is cyclic, as well.

This theorem has been proven by Hulanicki and Pytlik in [41. In fact, they showed it for first countable locally compact groups.

Recall that irreducible representations are cyclic.

Corollary 4.5. Let $\chi \in \hat{H}$ be an irreducible representation. Then, $\pi=\operatorname{ind}_{H}^{G}(\chi)$ can be decomposed into a direct sum $\pi=\pi_{1} \oplus \pi_{2}$ such that $\pi_{1}$ is a subrepresentation of the left regular representation $\lambda_{G}$, and $\pi_{2}$ and $\lambda_{G}$ are disjoint.

It is well-known that if $\chi$ is a subrepresentation of $\lambda_{H}$, then $\operatorname{ind}_{H}^{G}(\chi)$ is a subrepresentation of $\lambda_{G}$. This is due to

$$
\lambda_{G}=\operatorname{ind}_{H}^{G}\left(\lambda_{H}\right)=\operatorname{ind}_{H}^{G}\left(\chi \oplus \chi^{\prime}\right)=\operatorname{ind}_{H}^{G}(\chi) \oplus \operatorname{ind}_{H}^{G}\left(\chi^{\prime}\right),
$$

where $\chi^{\prime}$ is the orthogonal complement of $\chi$. On the other hand, if $\chi$ and $\lambda_{H}$ are disjoint, then $\operatorname{ind}_{H}^{G}(\chi)$ are not necessarily disjoint as the following example shows.

Example 4.6. Let

$$
G_{\text {aff }}(\mathbb{R})=\mathbb{R} \rtimes \mathbb{R}_{\neq 0}=\left\{(b, a) \mid a \in \mathbb{R}_{\neq 0}, b \in \mathbb{R}\right\}
$$

be the affine group of the real line and let

$$
H=\mathbb{R} \rtimes \mathbb{R}_{\neq 0}=\{(b, 1) \mid b \in \mathbb{R}\} .
$$

Then for any nontrivial irreducible representation $\chi \in \hat{H}, \operatorname{ind}_{H}^{G}(\chi)$ is square-integrable (cf. the classical continuous wavelet transformation, Chp. 3.1.1). 


\subsection{Continuous wavelet transformations on unimodular groups}

Firstly, we target "good" representations. Let $G$ be a second countable locally compact group and suppose that its left regular representation $\lambda_{G}$ is type I. Let $\pi$ be a representation of type I, let

$$
\pi \cong \int_{\hat{G}}^{\oplus} m_{\pi}(\sigma) \sigma \mathrm{d} \mu(\sigma)
$$

be its central decomposition, and suppose that $\mu$ is absolutely continuous with respect to the Plancherel measure $\mu_{G}$. If $G$ is nonunimodular, then $\pi$ is square-integrable by Thm. 3.16. If $G$ is unimodular, then there are a few things that can go wrong. On the one hand, it is possible that

$$
m_{\pi}(\sigma)>\operatorname{dim}(\sigma)
$$

for all $\sigma$ in a subset of positive Plancherel measure. In that case, $\pi$ is not cyclic and, in particular, not a subrepresentation of $\lambda_{G}$. On the other hand, it is possible that

$$
\int_{\hat{G}} m_{\pi}(\sigma) \mathrm{d} \mu_{G}(\sigma)=\infty .
$$

In either case the representation $\pi$ is "too large". This is the situation we want to address now. The basic idea is to split $\pi$ into smaller pieces which are square-integrable. Then for each of the subrepresentations we can find an admissible vector. Taken together we get an admissible family of vectors, which defines a new approach to continuous wavelet transformations and generalizes the concept discussed in Chp. 3.2

Theorem 4.7. Let $G$ be a unimodular second countable locally compact group and suppose that $\lambda_{G}$ is type $I$. Let $\pi$ be a representation of type $I$, let

$$
\pi \cong \int_{\hat{G}}^{\oplus} m_{\pi}(\sigma) \sigma \mathrm{d} \mu(\sigma)
$$

be its central decomposition, and suppose that $\mu$ is absolutely continuous with respect to the Plancherel measure $\mu_{G}$.

Then there is a decomposition $\pi=\bigoplus_{n \in \mathbb{N}} \pi_{n}$ into a direct sum of subrepresentations such that $\pi_{n}$ admits an admissible vector for all $n \in \mathbb{N}$.

Proof. By Dixmier [21, Prop. 5.4.9] $\pi$ can be decomposed into a direct sum

$$
\pi=\bigoplus_{d \in I} d \cdot \rho_{d},
$$

where $I \subseteq \overline{\mathbb{N}}=\mathbb{N} \cup\{\infty\}$ and $\rho_{d}$ for $d \in I$ are multiplicity-free subrepresentations of $\pi$ which are pairwise disjoint. Since $I$ as well as the multiplicities $d \in I$ are countable, it 
is sufficient to prove the statement for multiplicity-free representations.

So assume that $\pi$ is multiplicity-free and let

$$
\pi \cong \int_{\hat{G}}^{\oplus} \sigma \mathrm{d} \mu(\sigma)
$$

be its central decomposition. Since $\mu$ is $\sigma$-finite (cf. Lem. 4.1), there exists a decomposition

$$
\hat{G}=\coprod_{l \in \mathbb{N}} E_{l}
$$

into a disjoint union of measurable subsets of finite Plancherel measure. Then, $\pi$ can be decomposed into the direct sum

$$
\pi \cong \bigoplus_{l \in \mathbb{N}} \int_{E_{l}}^{\oplus} \sigma \mathrm{d} \mu(\sigma) .
$$

By Thm. 3.16, each of the representations $\pi_{l}:=\int_{E_{l}}^{\oplus} \sigma \mathrm{d} \mu(\sigma)$ is square-integrable as

$$
\int_{E_{l}} 1 \mathrm{~d} \mu_{G}(\sigma)=\mu_{G}\left(E_{l}\right)<\infty
$$

and $m_{\pi_{l}}(\sigma) \leq 1$ for $\mu$-almost all $\sigma$.

With this decomposition we can generalize continuous wavelet transformations as follows.

Corollary 4.8. Let $G$ and $\pi$ be as in Thm. 4.7. Then there exists an admissible family $\Psi=\left\{\psi_{n}\right\}_{n \in \mathbb{N}} \subseteq \mathcal{H}_{\pi}$ for $\pi$. That is, the operator $W_{\Psi}: \mathcal{H}_{\pi} \rightarrow L^{2}(G \times \mathbb{N})$,

$$
W_{\Psi} f(a, n)=\left\langle\pi(a) \psi_{n}, f\right\rangle \quad \forall f \in \mathcal{H}_{\pi},
$$

is an isometry.

Proof. Let $\pi=\bigoplus_{n \in \mathbb{N}} \pi_{n}$ be the decomposition given in Thm. 4.7 and let $\left\{P_{n}\right\}_{n \in \mathbb{N}}$ be the orthogonal projections onto the Hilbert spaces of $\left\{\pi_{n}\right\}_{n \in \mathbb{N}}$. For all $n \in \mathbb{N}$, let $\psi_{n} \in P_{n}\left(\mathcal{H}_{\pi}\right)$ be an admissible vector for $\pi_{n}$ and let $\Psi=\left\{\psi_{n}\right\}_{n \in \mathbb{N}}$. Then $W_{\Psi}$ satisfies

$$
\sum_{n \in \mathbb{N}} \int_{G}\left|\left\langle\pi(a) \psi_{n}, f\right\rangle\right|^{2} \mathrm{~d} a=\sum_{n \in \mathbb{N}}\left\|P_{n} f\right\|^{2}=\|f\|^{2}
$$

for all $f \in \mathcal{H}_{\pi}$.

\subsection{Continuous wavelet transformations on homogeneous spaces}

We now deal with the question of continuous wavelet transformations (in a generalized sense) on homogeneous spaces. As we saw before, having good knowledge of 
the Plancherel measure of the group and the central measure of the representation of interest is crucial for generalizing continuous wavelet transformations.

As shown in Thm. 3.16, there are two reasons why a continuous wavelet transformation in the sense of Chp. 3.2 does not exist. The first one is that the representation is "too large" as discussed in Chp. 4.2. In that case, enlarging the domain of integration from $G$ to the larger spaces $G \times \mathbb{N}$ (or $G \times \mathbb{R}$ as in Chp. 3.1.6) seems to be the right way to deal with this situation. The second reason is that the representation is disjoint to the left regular representation. In that case, the central measure is singular with respect to the Plancherel measure. Therefore, instead of using the Haar measure, it might be a good strategy to choose a measure which is singular with respect to the Haar measure. In fact, this is exactly what Perelomov as well as de Bièvre do in their approaches presented in Chp. 3.1 .3 and Chp. 3.1.4.

However, there is a problem with this approach. Suppose that $\left(\pi, \mathcal{H}_{\pi}\right)$ is a unitary representation of a locally compact group $G$ and $\mu$ is a measure on $G$ such that, for a fixed vector $\psi \in \mathcal{H}_{\pi}$, all $f \in \mathcal{H}_{\pi}$ satisfy

$$
\|f\|^{2}=\int_{G}\left|C_{\psi} f(a)\right|^{2} \mathrm{~d} \mu(a),
$$

where $C_{\psi} f(a)=\langle\pi(a) \psi, f\rangle$. Then, for $b \in G$, we get

$$
\begin{aligned}
\int_{G}\left|C_{\psi} f(a)\right|^{2} \mathrm{~d} \mu(a) & =\|f\|^{2}=\|\pi(b) f\|^{2}=\int_{G}\left|C_{\psi} f\left(b^{-1} a\right)\right|^{2} \mathrm{~d} \mu(a) \\
& =\int_{G}\left|C_{\psi} f(a)\right|^{2} \mathrm{~d} \mu_{b}(a),
\end{aligned}
$$

where $\mu_{b}$ is the translated measure. In most cases $\mu$ will not be $G$-invariant, meaning $\mu_{b} \neq \mu$. So, in that sense we lose $G$-invariance. Unfortunately, this is a trade-off which is inevitable. At least the set of measures making eq. (4.1) valid is $G$-invariant.

\subsubsection{Semidirect products}

Representations defined on function spaces on homogeneous spaces often appear when constructing irreducible representations of a group using the induction procedure. One of the most important examples is Mackey's machine, which is used to construct (almost) all irreducible representations of a given group and helps to understand the structure of the dual of the group. In the following we want to use these results to construct generalized continuous wavelet transformations for semidirect products of the form $\mathbb{R}^{n} \rtimes H$ with $H \leq \operatorname{Gl}(n)$, which play an important role in many applications. Examples coming from physics are the Euclidean group, the Galilei group, and the Poincaré group.

Let $n \in \mathbb{N}$. Cartan's Closed-subgroup Theorem states that every closed subgroup of $\operatorname{Gl}(n)$ is a Lie group which acts smoothly on $\mathbb{R}^{n}$. Those are the assumptions that we will need in the following. So, more generally, let $H$ be a Lie group which acts smoothly 
on the abelian Lie group $\mathbb{R}^{n}$ and let $G=\mathbb{R}^{n} \rtimes H$ be the semidirect product of $\mathbb{R}^{n}$ and $H$. By representing $G$ in the form

$$
G=\left\{(v, h) \mid v \in \mathbb{R}^{n}, h \in H\right\}
$$

multiplication and inversion are given by

$$
\begin{aligned}
\left(v_{1}, h_{1}\right)\left(v_{2}, h_{2}\right) & =\left(v_{1}+h_{1} \cdot v_{2}, h_{1} h_{2}\right), \\
(v, h)^{-1} & =\left(-h^{-1} \cdot v, h^{-1}\right),
\end{aligned}
$$

for $\left(v_{1}, h_{1}\right),\left(v_{2}, h_{2}\right),(v, h) \in G$. Every element $a \in G$ can be uniquely written as a product $a=(0, h)\left(v, e_{H}\right)=(h . v, h)$, where $e_{H}$ is the identity element in $H$. The left Haar measure of $G$ has the form

$$
\int_{G} \varphi(a) \mathrm{d} a=\int_{H} \int_{\mathbb{R}^{n}} \varphi(h . v, h) \mathrm{d} v \mathrm{~d} h, \quad \forall \varphi \in C_{c}(G),
$$

where $\mathrm{d} v$ is the Lebesgue measure on $\mathbb{R}^{n}$ and $\mathrm{d} h$ is the Haar measure on $H$. Of course, one could use the decomposition $a=\left(v, e_{H}\right)(0, h)=(v, h)$, as well. Then the Haar measure has the form

$$
\int_{G} \varphi(a) \mathrm{d} a=\int_{H} \int_{\mathbb{R}^{n}} \varphi(v, h)|\operatorname{det}(h)|^{-1} \mathrm{~d} v \mathrm{~d} h, \quad \forall \varphi \in C_{c}(G),
$$

with $\operatorname{det}(h):=\operatorname{det}(v \mapsto h \cdot v)$. Both realizations are equivalent. The first one, however, will turn out to be more comfortable.

The action of $H$ on $\mathbb{R}^{n}$ induces an action on $\widehat{\mathbb{R}^{n}}$ defined by

$$
\chi \mapsto h \cdot \chi, \quad h \cdot \chi(v)=\chi\left(h^{-1} \cdot v\right), \quad h \in H, \chi \in \widehat{\mathbb{R}^{n}}, v \in \mathbb{R}^{n} .
$$

Since there exists the one-to-one correspondence between $\left(\mathbb{R}^{n}\right)^{*}$ and $\widehat{\mathbb{R}^{n}}$, given by

$$
k \mapsto \chi_{k}, \quad \chi_{k}(v)=e^{2 \pi i k(v)}, \quad v \in \mathbb{R}^{n},
$$

the action of $H$ on $\widehat{\mathbb{R}^{n}}$ goes over into an action on $\left(\mathbb{R}^{n}\right)^{*}$ given by

$$
k \mapsto h . k, \quad h . k(v)=k\left(h^{-1} . v\right), \quad h \in H, k \in\left(\mathbb{R}^{n}\right)^{*}, v \in \mathbb{R}^{n} .
$$

For $k \in\left(\mathbb{R}^{n}\right)^{*}$, denote its orbit by $\mathcal{O}_{k} \subseteq\left(\mathbb{R}^{n}\right)^{*}$ and its stabilizer by $H_{k} \leq H$. Let $\tau$ be a representation of $H_{k}$ and define the representation $\sigma=\chi_{k} \times \tau$ of $G_{k}:=\mathbb{R}^{n} \rtimes H_{k}$ by

$$
\sigma(v, h)=\chi_{k}(v) \tau(h), \quad \forall(v, h) \in G_{k} .
$$


$\sigma$ is well-defined as

$$
\begin{aligned}
\sigma\left(\left(v_{1}, h_{1}\right)\left(v_{2}, h_{2}\right)\right) & =\sigma\left(v_{1}+h_{1}\left(v_{2}\right), h_{1} h_{2}\right)=\chi_{k}\left(v_{1}\right) \tau\left(h_{1}\right) \underbrace{\chi_{k}\left(h_{1}\left(v_{2}\right)\right)}_{=\chi_{k}\left(v_{2}\right)} \tau\left(h_{2}\right) \\
& =\sigma\left(v_{1}, h_{1}\right) \sigma\left(v_{2}, h_{2}\right), \\
\sigma\left((v, h)^{-1}\right) & =\sigma\left(-h^{-1} \cdot v, h^{-1}\right)=\underbrace{\chi_{k}\left(-h^{-1} \cdot v\right)}_{=\chi_{k}(-v)} \tau\left(h^{-1}\right)=\chi_{k}(v)^{*} \tau(h)^{*} \\
& =\sigma(v, h)^{*},
\end{aligned}
$$

for $\left(v_{1}, h_{1}\right),\left(v_{2}, h_{2}\right),(v, h) \in G_{k}$.

Using these notions the irreducible representations of $G$ can be characterized with the help of the following result, which is known as Mackey's machine for semidirect products (cf. Folland [30, Thm. 6.43]).

Theorem 4.9 (Mackey's machine for semidirect products).

Let $G=\mathbb{R}^{n} \rtimes H$ be a semidirect product of $\mathbb{R}^{n}$ and a Lie group $H$ which acts smoothly on $\mathbb{R}^{n}$. Suppose that the $H$-orbits in $\left(\mathbb{R}^{n}\right)^{*}$ are locally closed.

- For all $k \in\left(\mathbb{R}^{n}\right)^{*}$ and $\tau \in \hat{H}_{k}$, $\operatorname{ind}_{G_{k}}^{G}\left(\chi_{k} \times \tau\right)$ is an irreducible representation of G.

- Every irreducible representation of $G$ is of this form.

- Two representations $\operatorname{ind}_{G_{k}}^{G}\left(\chi_{k} \times \tau\right)$ and $\operatorname{ind}_{G_{k^{\prime}}}^{G}\left(\chi_{k^{\prime}} \times \tau^{\prime}\right)$ are equivalent if and only if $k$ and $k^{\prime}$ belong to the same orbit, say $k^{\prime}=m . k$ for some $m \in H$, and $h \mapsto \tau(h)$ and $h \mapsto \tau^{\prime}\left(m h m^{-1}\right)$ are equivalent representations of $H_{k}=m^{-1} H_{k^{\prime}} m$.

In 4], Aniello et al. study under which conditions irreducible representations of semidirect products are square-integrable. Their main theorem (cf. [4, Thm. 2]) applied to the context we have here reads as follows.

Theorem 4.10. Let $G=\mathbb{R}^{n} \rtimes H$ be a semidirect product of $\mathbb{R}^{n}$ and a Lie group $H$ which acts smoothly on $\mathbb{R}^{n}$. Let $k \in\left(\mathbb{R}^{n}\right)^{*}$ and $\tau \in \hat{H}_{k}$.

Then, $\operatorname{ind}_{G_{k}}^{G}\left(\chi_{k} \times \tau\right)$ is square-integrable if and only if $\mathcal{O}_{k} \subseteq\left(\mathbb{R}^{n}\right)^{*}$ has positive Lebesgue measure and $\tau$ is square-integrable.

So when dealing with irreducible representations of semidirect products $G$ there are two reasons why a given representation $\operatorname{ind}_{G_{k}}^{G}\left(\chi_{k} \times \tau\right)$ is not square-integrable. The first one is that the orbit $\mathcal{O}_{k} \subseteq\left(\mathbb{R}^{n}\right)^{*}$ is a null set and the second one is that $\tau \in \hat{H}_{k}$ is not square-integrable. The latter situation is a very challenging problem as $\hat{H}_{k}$ still can have a complicated structure. However, the former case seems to be much easier since $\left(\mathbb{R}^{n}\right)^{*} \cong \mathbb{R}^{n}$ has a well-known structure and can even be considered as a smooth manifold.

From now on, let $\eta \in\left(\mathbb{R}^{n}\right)^{*}$ be a fixed element and let $\tau$ be a fixed representation of $H_{\eta}$. Before proceeding with the discussion of square-integrability, let us have a closer 
look at the representation $\operatorname{ind}_{G_{\eta}}^{G}\left(\chi_{\eta} \times \tau\right)$. In contrast to Thm. 4.9, we do not assume that the orbit $\mathcal{O}_{\eta}$ of $\eta$ is locally closed in $\left(\mathbb{R}^{n}\right)^{*} . \mathcal{O}_{\eta}$ is endowed with the quotient topology, which does not necessarily coincide with the subspace topology. Moreover, $\mathcal{O}_{\eta}$ is endowed with the quotient smooth structure (as a homogeneous $H$-manifold) but, in general, it is not a submanifold of $\left(\mathbb{R}^{n}\right)^{*}$.

Let

$$
\mathcal{O}_{\eta} \rightarrow H, \quad k \mapsto h_{k},
$$

be a regular Borel section, meaning that $h_{k} . \eta=k$ for all $k \in \mathcal{O}_{\eta}$ and there exists a quasi-invariant Radon measure $\nu_{\eta}$ on $\mathcal{O}_{\eta}$ associated to the cross-section $k \mapsto h_{k}$ (cf. Lem. 2.33). Then every element $h \in H$ can be uniquely written as a product $h=h_{k} m$, where $k \in \mathcal{O}_{\eta}$ and $m \in H_{\eta}$, and integration on $H$ against the Haar measure can be decomposed into

$$
\begin{array}{rlr}
\int_{H} \varphi(h) \mathrm{d} h & =\int_{\mathcal{O}_{\eta}} \int_{H_{\eta}} \frac{\Delta_{H}(m)}{\Delta_{H_{\eta}}(m)} \varphi\left(h_{k} m\right) \mathrm{d} m \mathrm{~d} \nu_{\eta}(k) & \\
& =\int_{\mathcal{O}_{\eta}} \int_{H_{\eta}} \delta_{H_{\eta}}^{H}(m)^{2} \varphi\left(h_{k} m\right) \mathrm{d} m \mathrm{~d} \nu_{\eta}(k), & \forall \varphi \in C_{c}(G)
\end{array}
$$

for $\delta_{H_{\eta}}^{H}: H_{\eta} \rightarrow \mathbb{R}_{>0}$

$$
\delta_{H_{\eta}}^{H}(m)=\sqrt{\frac{\Delta_{H}(m)}{\Delta_{H_{\eta}}(m)}} .
$$

The representation

$$
\pi \cong \operatorname{ind}_{G_{\eta}}^{G}\left(\chi_{\eta} \times \tau\right)
$$

can be realized on the Hilbert space $\mathcal{H}_{\pi}=L^{2}\left(H, H_{\eta}, \delta_{H_{\eta}}^{H} \tau\right)$ given by

$$
\begin{aligned}
f(h m) & =\delta_{H_{\eta}}^{H}\left(m^{-1}\right) \tau\left(m^{-1}\right) f(h), & \forall h \in H, m \in H_{\chi}, \\
\langle f, g\rangle & =\int_{\mathcal{O}_{\eta}}\left\langle f\left(h_{k}\right), g\left(h_{k}\right)\right\rangle_{\tau} \mathrm{d} \nu_{\eta}(k), &
\end{aligned}
$$

and has the form

$$
\begin{aligned}
{\left[\pi\left(v, e_{H}\right) f\right]\left(h^{\prime}\right) } & =e^{2 \pi i\left(h^{\prime} . \eta\right)(v)} f\left(h^{\prime}\right), \\
{[\pi(0, h) f]\left(h^{\prime}\right) } & =f\left(h^{-1} h^{\prime}\right) .
\end{aligned}
$$

Recall that $f \in L^{2}\left(H, H_{\eta}, \delta_{H_{\eta}}^{H} \tau\right)$ is uniquely determined by the values of

$$
\Theta(f): k \mapsto f\left(h_{k}\right) \in L^{2}\left(\mathcal{O}_{\eta}, \mathcal{H}_{\tau}, \nu_{\eta}\right)
$$

and the map $\Theta: L^{2}\left(H, H_{\eta}, \delta_{H_{\eta}}^{H} \tau\right) \rightarrow L^{2}\left(\mathcal{O}_{\eta}, \mathcal{H}_{\tau}, \nu_{\eta}\right)$ is an isometric isomorphism. 
The coefficient functions of $\pi$ are given by

$$
\begin{aligned}
C_{f} g(h(v), h) & =\langle\pi(h(v), h) f, g\rangle=\left\langle\pi\left(v, e_{H}\right) f, \pi(0, h)^{*} g\right\rangle \\
& =\int_{\mathcal{O}_{\eta}} e^{-2 \pi i\left(h_{k} \cdot \eta\right)(v)}\left\langle f\left(h_{k}\right), g\left(h h_{k}\right)\right\rangle_{\tau} \mathrm{d} \nu_{\eta}(k) \\
& =\int_{\mathcal{O}_{\eta}} e^{-2 \pi i k(v)}\left\langle f\left(h_{k}\right), g\left(h h_{k}\right)\right\rangle_{\tau} \mathrm{d} \nu_{\eta}(k) .
\end{aligned}
$$

Note that the left-hand side of eq. (4.5) is a bounded, continuous function in $v \in \mathbb{R}^{n}$ and, therefore, it can be considered as a regular tempered distribution on $\mathbb{R}^{n}$. The right-hand side is an inverse Fourier transform of a finite signed measure on $\left(\mathbb{R}^{n}\right)^{*}$, which is bounded by

$$
\int_{\mathcal{O}_{\eta}}\left|\left\langle f\left(h_{k}\right), g\left(h h_{k}\right)\right\rangle_{\tau}\right| \mathrm{d} \nu_{\eta}(k) \leq\|f\| \cdot\left\|\pi(0, h)^{*} g\right\|=\|f\| \cdot\|g\| .
$$

If $\mathcal{O}_{\eta}$ is a null set, then $\nu_{\eta}$ is not absolutely continuous with respect to the Lebesgue measure. From the theory of tempered distributions (cf. Lem. B.1 and Lem. B.2) it follows that, for all $h \in H$,

$$
\varphi \mapsto \int_{\mathcal{O}_{\eta}} \varphi(k)\left\langle f\left(h_{k}\right), g\left(h h_{k}\right)\right\rangle_{\tau} \mathrm{d} \nu_{\eta}(k)
$$

is not a regular distribution, hence not in $L^{2}\left(\left(\mathbb{R}^{n}\right)^{*}\right)$, and, therefore, the map $v \mapsto$ $C_{f} g(h(v), h)$ is not in $L^{2}\left(\mathbb{R}^{n}\right)$ (unless it is constant zero). In particular, a nonzero coefficient function $C_{f} g$ cannot be in $L^{2}(G)$. It turns out that there are directions in $\mathbb{R}^{n}$ in which $v \mapsto C_{f} g(h(v), h)$ is not decaying fast enough. Hence, it is important to exclude those directions when integrating. The strategy we want to use is inspired by the construction of de Bièvre [20] and is described in the following example.

Example 4.11. Let $G=\mathbb{R}^{2} \rtimes \mathrm{SO}(2)$. The group $H=\mathrm{SO}(2)$ acts on $\left(\mathbb{R}^{2}\right)^{*}$ by

$$
\text { A. } k=k A^{-1}=k A^{t}, \quad \text { where } k=\left(k_{1}, k_{2}\right), A=\left(\begin{array}{cc}
\cos \alpha & -\sin \alpha \\
\sin \alpha & \cos \alpha
\end{array}\right) .
$$

There are two different kinds of $\mathrm{SO}(2)$-orbits in $\left(\mathbb{R}^{2}\right)^{*}$. For $\eta=0, \mathcal{O}_{\eta}$ only consists of one point and, for $\eta \neq 0, \mathcal{O}_{\eta}$ forms a circle.

- If $\eta=0 \in\left(\mathbb{R}^{2}\right)^{*}$ then the stabilizer $H_{\eta}$ is the whole group $H$ and $G_{\eta}:=\mathbb{R}^{2} \rtimes H_{\eta}=$ $G$. Every irreducible representation $\tau_{n}$ of $H$,

$$
\tau_{n}\left(\begin{array}{cc}
\cos \alpha & -\sin \alpha \\
\sin \alpha & \cos \alpha
\end{array}\right)=e^{i n \alpha}
$$

yields an irreducible representation $\pi_{0, n}=\chi_{0} \times \tau_{n}$ of $G$, given by

$$
\pi_{0, n}(v, A)=\tau_{n}(A) \in \mathrm{U}(1),
$$


for all $(v, A) \in G$. The coefficient functions of $\pi_{0, n}$ have the form

$$
C_{f} g(A v, A)=\overline{\tau_{n}(A) f} \cdot g \quad \forall f, g \in \mathbb{C}
$$

and do not depend on $v \in \mathbb{R}^{2}$. In particular $v \mapsto C_{f} g(A v, A)$ does not decay as $\|v\| \rightarrow \infty$. So instead of integrating over $G$ we only integrate over $H$ and get

$$
\int_{H}\left|C_{f} g(A v, A)\right|^{2} \mathrm{~d} A=\int_{0}^{2 \pi}\left|e^{-i n \alpha} \bar{f} \cdot g\right|^{2} \frac{\mathrm{d} \alpha}{2 \pi}=|f|^{2} \cdot|g|^{2} .
$$

Therefore, $\pi_{0, n}$ is not square-integrable with respect to the Haar measure of $G$ but with respect to the measure

$$
\varphi \mapsto \int_{H} \int_{\mathbb{R}^{2}} \varphi(A v, A) \mathrm{d} \delta_{v_{0}}(v) \mathrm{d} A, \quad \forall \varphi \in C_{c}(G),
$$

where $\delta_{v_{0}}$ is the Dirac measure at some arbitrary point $v_{0} \in \mathbb{R}^{2}$. Then we get

$$
\int_{H} \int_{\mathbb{R}^{2}}\left|C_{f} g(A v, A)\right|^{2} \mathrm{~d} \delta_{v_{0}}(v) \mathrm{d} A=|g|^{2}
$$

if $f$ satisfies the admissibility condition $|f|^{2}=1$.

- If $\eta \in\left(\mathbb{R}^{2}\right)^{*} \backslash\{0\}$ then the situation is more interesting. Now the orbits are circles of the form

$$
\mathcal{O}_{\eta}=\{(|\eta| \cos \kappa,|\eta| \sin \kappa) \mid \kappa \in \mathbb{R}\} \subseteq\left(\mathbb{R}^{2}\right)^{*}
$$

and the stabilizers are trivial. The representations $\pi_{\eta} \cong \operatorname{ind}_{\mathbb{R}^{2}}^{G}\left(e^{2 \pi i \eta}\right)$ are defined on $L^{2}\left(\mathcal{O}_{\eta}, \mathcal{H}_{\tau}, \nu_{\eta}\right)$ and are given by

$$
\left[\pi_{\eta}(v, A) f\right](k)=e^{2 \pi i k(v)} f(k A) \quad f \in L^{2}\left(\mathcal{O}_{\eta}, \mathcal{H}_{\tau}, \nu_{\eta}\right),(v, A) \in G .
$$

The coefficient functions of $\pi_{\eta}$ have the form

$$
\begin{aligned}
C_{f} g(A v, A) & =\int_{\mathcal{O}_{\eta}} e^{-2 \pi i k(v)} \overline{f(k)} g\left(k A^{t}\right) \mathrm{d} \nu_{\eta}(k) \\
& =\int_{0}^{2 \pi} e^{-2 \pi i|\eta|\left(v_{1} \cos \kappa+v_{2} \sin \kappa\right)} \overline{f(k)} g\left(k A^{t}\right) \mathrm{d} \kappa,
\end{aligned}
$$

where $k=(|\eta| \cos \kappa,|\eta| \sin \kappa)$ and $v=\left(v_{1}, v_{2}\right)^{t}$.

Again $\pi_{\eta}$ is not square-integrable as $\mathcal{O}_{\eta}$ is not open. The modulus of the coefficient functions is given by

$$
\begin{gathered}
\left|C_{f} g(A v, A)\right|^{2}=\int_{0}^{2 \pi} \mathrm{d} \kappa \int_{0}^{2 \pi} \mathrm{d} \lambda e^{2 \pi i|\eta|\left(v_{1}(\cos \lambda-\cos \kappa)+v_{2}(\sin \lambda-\sin \kappa)\right)} \\
\cdot \overline{f(k)} f(l) g\left(k A^{t}\right) \overline{g\left(l A^{t}\right)}
\end{gathered}
$$


where $l=(|\eta| \cos \lambda,|\eta| \sin \lambda) \in \mathcal{O}_{\eta}$. Instead of integrating $v$ over $\mathbb{R}^{2}$ one can try to integrate over a one-dimensional subspace, say $\left\{\left(v_{1}, 0\right)^{t} \mid v_{1} \in \mathbb{R}\right\}$. Then formally one gets

$$
\begin{aligned}
\int_{\mathbb{R}} e^{2 \pi i|\eta| v_{1}(\cos \lambda-\cos \kappa)} \mathrm{d} v_{1} & =\delta_{0}(|\eta|(\cos \lambda-\cos \kappa)) \\
& =\frac{1}{|\eta||\sin (\kappa)|}\left(\delta_{2 \pi \mathbb{Z}}(\kappa-\lambda)+\delta_{2 \pi \mathbb{Z}}(\kappa+\lambda)\right),
\end{aligned}
$$

where $\delta_{2 \pi \mathbb{Z}}=\sum_{m \in \mathbb{Z}} \delta_{2 \pi m}$ and $\delta_{2 \pi m}$ is the Dirac distribution. Assume that the function $f$ is supported on the half circle

$$
\{(|\eta| \cos \kappa,|\eta| \sin \kappa) \mid 0<\kappa<\pi\} \subseteq \mathcal{O}_{\eta} .
$$

Then $\kappa+\lambda \in(0,2 \pi)$ for all $\kappa, \lambda \in(0, \pi)$ and $\delta_{2 \pi \mathbb{Z}}(\kappa+\lambda) \overline{f(k)} f(l)$ will always be zero. Consequently, for $v=\left(\begin{array}{c}v_{1} \\ 0\end{array}\right)$ we get

$$
\begin{aligned}
\int_{\mathbb{R}}\left|C_{f} g(A v, A)\right|^{2} \mathrm{~d} v_{1} & =\int_{0}^{2 \pi} \mathrm{d} \kappa \int_{0}^{2 \pi} \mathrm{d} \lambda \frac{\delta_{2 \pi \mathbb{Z}}(\kappa-\lambda)}{|\eta||\sin (\kappa)|} \overline{f(k)} f(l) g\left(k A^{t}\right) \overline{g\left(l A^{t}\right)} \\
& =\int_{0}^{2 \pi} \mathrm{d} \kappa \frac{1}{|\eta||\sin (\kappa)|}|f(k)|^{2}\left|g\left(k A^{t}\right)\right|^{2}
\end{aligned}
$$

Finally integrating over $H$ yields

$$
\begin{aligned}
\int_{H} \int_{\mathbb{R}}\left|C_{f} g(A v, A)\right|^{2} \mathrm{~d} v_{1} \mathrm{~d} A & =\int_{H} \mathrm{~d} A \int_{0}^{2 \pi} \mathrm{d} \kappa \frac{1}{|\eta||\sin (\kappa)|}|f(k)|^{2}\left|g\left(k A^{t}\right)\right|^{2} \\
& =\int_{0}^{2 \pi} \frac{\mathrm{d} \lambda}{2 \pi} \int_{0}^{2 \pi} \mathrm{d} \kappa \frac{1}{|\eta||\sin (\kappa)|}|f(k)|^{2}|g(l)|^{2} \\
& =\int_{0}^{2 \pi} \mathrm{d} \kappa \frac{1}{2 \pi|\eta||\sin (\kappa)|}|f(k)|^{2}\|g\|^{2}
\end{aligned}
$$

where we substituted $k A^{t}$ for $l=(|\eta| \cos \lambda,|\eta| \sin \lambda)$.

It follows that $\pi_{\eta}$ is square-integrable with respect to the measure

$$
\varphi \mapsto \int_{H} \int_{\mathbb{R}^{2}} \varphi(A v, A) \mathrm{d} v_{1} \mathrm{~d} \delta_{0}\left(v_{2}\right) \mathrm{d} A .
$$

If $f$ satisfies the admissibility condition

$$
\int_{0}^{2 \pi} \mathrm{d} \kappa \frac{|f(k)|^{2}}{2 \pi|\eta||\sin (\kappa)|}=1
$$

then we get

$$
\int_{H} \int_{\mathbb{R}}\left|C_{f} g(A v, A)\right|^{2} \mathrm{~d} v_{1} \mathrm{~d} A=\|g\|^{2} .
$$

The idea of Ex. 4.11 leads to the following definition and theorem. 
Definition 4.12. Let $G$ be a second countable locally compact group and let $(\pi, \mathcal{H})$ be a representation of $G$. For a Radon measure $\mu$ on $G$ the representation $\pi$ is called $\mu$-square-integrable if there exists a vector $\psi \in \mathcal{H}$ such that

$$
\int_{G}|\langle\pi(v) \psi, f\rangle|^{2} \mathrm{~d} \mu(v)=\|f\|^{2} \quad \forall f \in \mathcal{H}
$$

In that case, the vector $\psi$ is called $\mu$-admissible.

Theorem 4.13. Let $G=\mathbb{R}^{n} \rtimes H$ be a semidirect product of $\mathbb{R}^{n}$ and a Lie group $H$ which acts smoothly on $\mathbb{R}^{n}$. Let $\eta \in\left(\mathbb{R}^{n}\right)^{*}$ and let $\tau$ be a square-integrable representation of $H_{\eta}$.

Then there exists a Radon measure $\mu$ on $G$ such that $\left.\pi=\operatorname{ind}_{\mathbb{R}^{n} \rtimes H_{\eta}}^{\mathbb{R}^{n} \rtimes \chi_{\eta}} \times \tau\right)$ is $\mu$-squareintegrable. $\mu$ has the form

$$
\int_{G} \varphi(a) \mathrm{d} \mu(a)=\int_{H} \int_{W} \varphi(h(w), h) \mathrm{d} w \mathrm{~d} h, \quad \forall \varphi \in C_{c}(G),
$$

where $W \subseteq \mathbb{R}^{n}$ is a linear subspace of dimension $\operatorname{dim}(W)=\operatorname{dim}\left(H / H_{\eta}\right)$.

Before proving Thm. 4.13, let us go back to eq. 4.7 in Ex. 4.11, where we used that

$$
\int_{\mathbb{R}} e^{2 \pi i|\eta| v_{1}(\cos \lambda-\cos \kappa)} \mathrm{d} v_{1}=\frac{1}{|\eta||\sin (\kappa)|}\left(\delta_{2 \pi \mathbb{Z}}(\kappa-\lambda)+\delta_{2 \pi \mathbb{Z}}(\kappa+\lambda)\right) .
$$

To derive this equation, consider the integral

$$
\begin{aligned}
\int_{-\pi}^{\pi} e^{-2 \pi i|\eta| v_{1} \cos \kappa} \varphi(\kappa) \mathrm{d} \kappa & =\int_{-|\eta|}^{|\eta|} e^{-2 \pi i v_{1} y} \varphi(\arccos (y /|\eta|)) \frac{1}{\sqrt{|\eta|^{2}-y^{2}}} \mathrm{~d} y \\
& +\int_{-|\eta|}^{|\eta|} e^{-2 \pi i v_{1} y} \varphi(-\arccos (y /|\eta|)) \frac{1}{\sqrt{|\eta|^{2}-y^{2}}} \mathrm{~d} y .
\end{aligned}
$$

The right-hand side has the form of an inverse Fourier or Plancherel transform. So if we suppose that $\varphi$ is supported on $[0, \pi]$ (or $[-\pi, 0]$ respectively) and that

$$
y \mapsto \varphi(\arccos (y /|\eta|)) \frac{1}{\sqrt{|\eta|^{2}-y^{2}}}
$$

is in $L^{1}(\mathbb{R}) \cap L^{2}(\mathbb{R})$ then the left-hand side of eq. 4.9 is equal to the inverse Plancherel transform

$$
\int_{-\pi}^{\pi} e^{-2 \pi i|\eta| v_{1} \cos \kappa} \varphi(\kappa) \mathrm{d} \kappa=\int_{-|\eta|}^{|\eta|} e^{-2 \pi i v_{1} y} \varphi(\arccos (y /|\eta|)) \frac{1}{\sqrt{|\eta|^{2}-y^{2}}} \mathrm{~d} y .
$$


By applying the Plancherel Theorem we get

$$
\begin{aligned}
& \int_{\mathbb{R}} \int_{-\pi}^{\pi} \int_{-\pi}^{\pi} e^{2 \pi i|\eta| v_{1}(\cos \lambda-\cos \kappa)} \varphi(\kappa) \overline{\varphi(\lambda)} \mathrm{d} \lambda \mathrm{d} \kappa \mathrm{d} v_{1} \\
&=\int_{\mathbb{R}}\left|\int_{-\pi}^{\pi} e^{-2 \pi i|\eta| v_{1} \cos \kappa} \varphi(\kappa) \mathrm{d} \kappa\right|^{2} \mathrm{~d} v_{1} \\
&=\int_{-|\eta|}^{|\eta|}\left|\varphi(\arccos (y /|\eta|)) \frac{1}{\sqrt{|\eta|^{2}-y^{2}}}\right|^{2} \mathrm{~d} y \\
&=\int_{-\pi}^{\pi}|\varphi(\kappa)|^{2} \frac{1}{|\eta|^{2}|\sin \kappa|^{2}}|\eta||\sin \kappa| \mathrm{d} \kappa \\
&=\int_{-\pi}^{\pi}|\varphi(\kappa)|^{2} \frac{1}{|\eta||\sin \kappa|} \mathrm{d} \kappa,
\end{aligned}
$$

which is exactly what we saw in eq. 4.8.

From the geometric point of view this result can be interpreted as follows. Define the subspaces $W \subseteq \mathbb{R}^{2}, W^{\perp}, T \subseteq\left(\mathbb{R}^{2}\right)^{*}$ by

$$
\begin{aligned}
W & =\left\{\left(\begin{array}{c}
v_{1} \\
0
\end{array}\right) \mid v_{1} \in \mathbb{R}\right\}, \\
W^{\perp} & =\left\{\left(0, k_{2}\right) \mid k_{2} \in \mathbb{R}\right\}, \\
T & =\left\{\left(k_{1}, 0\right) \mid k_{1} \in \mathbb{R}\right\} .
\end{aligned}
$$

Let $P:\left(\mathbb{R}^{2}\right)^{*} \rightarrow\left(\mathbb{R}^{2}\right)^{*}$,

$$
P:\left(k_{1}, k_{2}\right) \mapsto\left(k_{1}, 0\right),
$$

be the projection with image $T$ and kernel $W^{\perp}$. Note that, for all $w \in W$ and $k \in\left(\mathbb{R}^{n}\right)^{*}$, we get $k(w)=P(k)(w)$. The affine subspace

$$
(0,|\eta|)+T
$$

can be identified with the tangent space of $\mathcal{O}_{\eta}$ at $(0,|\eta|)$ embedded in $\left(\mathbb{R}^{2}\right)^{*}$, as shown in the sketch on the left-hand side in Fig. 4.1

By the properties of the tangent space, the projection $P$ induces a diffeomorphism $\Phi=\left.P\right|_{U}: U \rightarrow U_{T}$ from the open neighborhood

$$
U=\left\{\left(k_{1}, k_{2}\right) \in \mathcal{O}_{\eta} \mid k_{2}>0\right\} \subseteq \mathcal{O}_{\eta}
$$

of $(0,|\eta|)$ to the open subset

$$
U_{T}=\left\{\left(k_{1}, 0\right) \in T \mid k_{1} \in(-|\eta|,|\eta|)\right\} \subseteq T .
$$

(See right sketch in Fig. 4.1.)

If the function $f$ in eq. 4.6 is supported on $U$, then $k \mapsto \overline{f(k)} g\left(k A^{t}\right)$ is supported on 

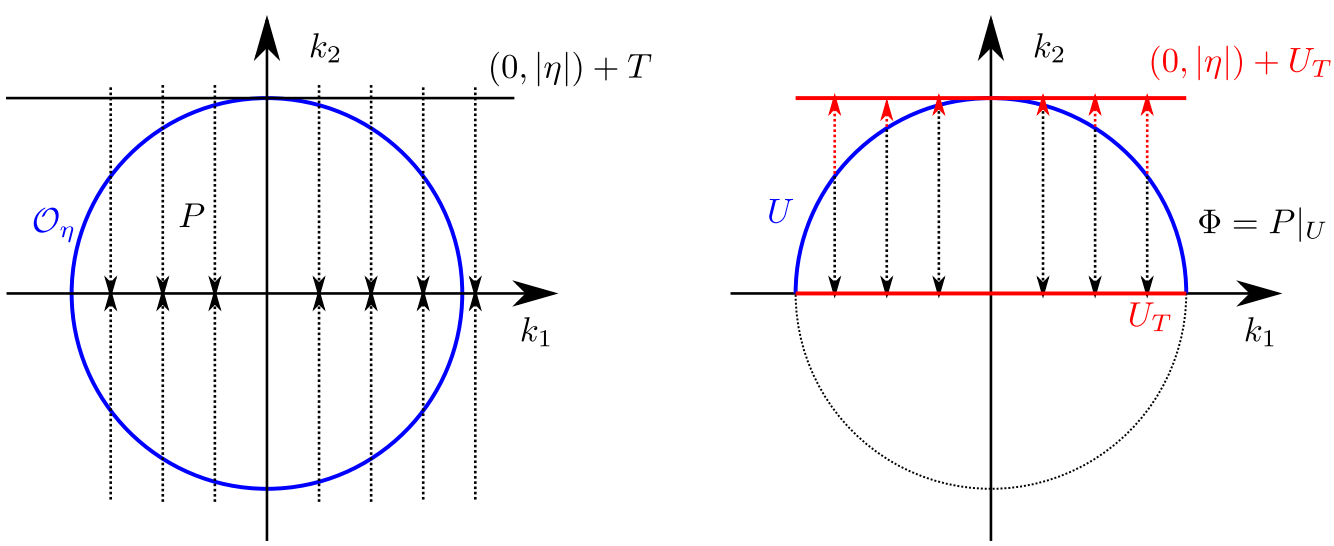

Figure 4.1: Sketch of the orbit $\mathcal{O}_{\eta}$ under the projection $P$ onto the space $T$.

$U$, as well, and we can use the diffeomorphism $\Phi: U \rightarrow U_{T}$ to parametrize $k \in U$ in terms of $t \in U_{T}$. Hence, we get

$$
\begin{aligned}
C_{f} g(A w, A) & =\int_{\mathcal{O}_{\eta}} e^{-2 \pi i k(w)} \overline{f(k)} g\left(k A^{t}\right) \mathrm{d} \nu_{\eta}(k) \\
& =\int_{U} e^{-2 \pi i \Phi(k)(w)} \overline{f(k)} g\left(k A^{t}\right) \mathrm{d} \nu_{\eta}(k) \\
& =\int_{U_{T}} e^{-2 \pi i t(w)} \overline{f\left(\Phi^{-1}(t)\right)} g\left(\Phi^{-1}(t) A^{t}\right) \mathrm{d} \nu_{\eta}\left(\Phi^{-1}(t)\right) \\
& =\int_{U_{T}} e^{-2 \pi i t(w)} \overline{f\left(\Phi^{-1}(t)\right)} g\left(\Phi^{-1}(t) A^{t}\right) \cdot \frac{\mathrm{d} \nu_{\eta}\left(\Phi^{-1}(t)\right)}{\mathrm{d} t} \mathrm{~d} t
\end{aligned}
$$

where we used that $\operatorname{supp}(f) \subseteq U$ and

$$
w \in W, \quad k(w)=P(k)(w)=\Phi(k)(w) \quad \text { for } k \in U .
$$

If the function

$$
t \mapsto \overline{f\left(\Phi^{-1}(t)\right)} g\left(\Phi^{-1}(t) A^{t}\right) \cdot \frac{\mathrm{d} \nu_{\eta}\left(\Phi^{-1}(t)\right)}{\mathrm{d} t}
$$

is in $L^{2}(T)$, then the Plancherel formula yields

$$
\begin{aligned}
\int_{W}\left|C_{f} g(A w, A)\right|^{2} \mathrm{~d} w & =\int_{U_{T}}\left|\overline{f\left(\Phi^{-1}(t)\right)} g\left(\Phi^{-1}(t) A^{t}\right) \cdot \frac{\mathrm{d} \nu_{\eta}\left(\Phi^{-1}(t)\right)}{\mathrm{d} t}\right|^{2} \mathrm{~d} t \\
& =\int_{U}\left|\overline{f(k)} g\left(k A^{t}\right)\right|^{2} \cdot \frac{\mathrm{d} \nu_{\eta}(k)}{\mathrm{d} \Phi(k)} \mathrm{d} \nu_{\eta}(k) .
\end{aligned}
$$

Again, this is what we saw in eq. (4.8).

With this construction in mind we prove the following auxiliary lemmas.

Lemma 4.14. Let $H$ be a Lie group which acts smoothly on $\mathbb{R}^{n}$ and let $\eta \in\left(\mathbb{R}^{n}\right)^{*}$.

- There exists an open neighborhood $U \subseteq H$ of $e_{H}$ such that $U . \eta$ is a submanifold of $\left(\mathbb{R}^{n}\right)^{*}$. 
- Moreover there exists a projection $P:\left(\mathbb{R}^{n}\right)^{*} \rightarrow\left(\mathbb{R}^{n}\right)^{*}$ onto the linear subspace $T=\operatorname{im}(P)$ such that the restriction

$$
\Phi=\left.P\right|_{U . \eta}: U . \eta \rightarrow U_{T}
$$

is a diffeomorphism, where $U_{T}:=P(U . \eta) \subseteq T$.

- The quasi-regular measure $\nu_{\eta}$ on $\mathcal{O}_{\eta}$ can be chosen such that it is smooth on U. $\eta$. In particular, there exists a smooth density function $\phi: U . \eta \rightarrow \mathbb{R}_{\geq 0}$ such that

$$
\phi(k)=\frac{\mathrm{d}\left(\Phi^{*} \nu_{U_{T}}\right)(k)}{\mathrm{d} \nu_{U . \eta}(k)},
$$

where $\nu_{U . \eta}$ is the restriction of $\nu_{\eta}$ to U. $\eta, \nu_{U_{T}}$ is the restriction of the Lebesgue measure of $T$ to $U_{T}$, and $\Phi^{*} \nu_{U_{T}}$ is the pullback of $\nu_{U_{T}}$ with respect to $\Phi$.

Proof. The map $H \rightarrow\left(\mathbb{R}^{n}\right)^{*}, h \mapsto h . \eta$, is smooth and has constant rank. By the Implicit Function Theorem there exists an open neighborhood $\tilde{U}$ of $e_{H} \in H$ such that $\tilde{U} . \eta$ is a submanifold of $\left(\mathbb{R}^{n}\right)^{*}$ and there exists a smooth local section $\tilde{h}: \tilde{U} . \eta \rightarrow H$, $k \mapsto \tilde{h}_{k}$.

Let $\bar{h}: \mathcal{O}_{\eta} \rightarrow H, k \mapsto \bar{h}_{k}$, be a regular Borel section. Recall that $\bar{h}$ is regular if $\bar{h}^{-1}(C)$ is a relatively compact subset of $H$ for all compact subsets $C \subseteq \mathcal{O}_{\eta}$. Let $U$ be a relatively compact, open subset of $\tilde{U}$ and define the cross-section $\mathcal{O}_{\eta} \rightarrow H, k \mapsto h_{k}$, by

$$
h_{k}=\left\{\begin{array}{ll}
\tilde{h}_{k}, & \text { if } k \in U . \eta \\
\bar{h}_{k}, & \text { if } k \notin U . \eta
\end{array} .\right.
$$

By construction, $k \mapsto h_{k}$ is a regular Borel section, which is smooth on $U$.

Let $\nu_{\eta}$ be the quasi-invariant measure on $\mathcal{O}_{\eta}$ associated to the cross-section $h$ (cf.

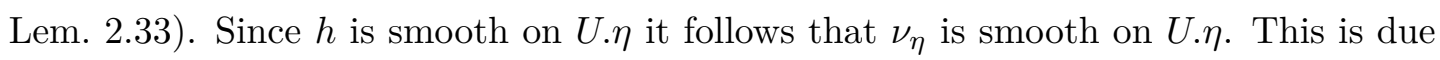
to the fact that we can use $h$ to smoothly transport $\mathrm{d} \nu_{\eta}(\eta)$ to $\mathrm{d} \nu_{\eta}(k)$ for all $k \in U . \eta$, meaning that $\left(h_{k}^{-1}\right)^{*}\left(\mathrm{~d} \nu_{\eta}(\eta)\right)=\mathrm{d} \nu_{\eta}(k)$.

Let $\eta+T$ be the tangent space of $U . \eta$ at $\eta$ embedded in $\left(\mathbb{R}^{n}\right)^{*}$ and let $P:\left(\mathbb{R}^{n}\right)^{*} \rightarrow\left(\mathbb{R}^{n}\right)^{*}$ be a projection with image $\operatorname{im}(P)=T$. When restricting $P$ to the $U . \eta$, the map $\Phi:=\left.P\right|_{U . \eta} \rightarrow T$ has full rank at $\eta$ as $\eta+T$ is the tangent space at $\eta$. By shrinking $U$ if necessary we can assume that $\Phi$ is a diffeomorphism. Let $\nu_{U . \eta}=\left.\nu_{\eta}\right|_{U . \eta}$ be the restriction of $\nu_{\eta}$ to $U . \eta$ and let $\nu_{U_{T}}$ be the restriction of the Lebesgue measure on $T$ to $U_{T}:=P(U . \eta)$. Then the pullback $\Phi^{*} \nu_{U_{T}}$ is a non-vanishing smooth measure on $U . \eta$ and, therefore, it is equivalent to $\nu_{U . \eta}$. In particular, there is a smooth density function

$$
\phi: U . \eta \rightarrow \mathbb{R}_{\geq 0}, \quad \phi(k)=\frac{\mathrm{d} \Phi^{*} \nu_{U_{T}}(k)}{\mathrm{d} \nu_{U . \eta}(k)}=\frac{\mathrm{d} \nu_{U_{T}}(\Phi(k))}{\mathrm{d} \nu_{U . \eta}(k)} .
$$


Using Lem. 4.14 we can show the following corollary.

Lemma 4.15. Let $G=\mathbb{R}^{n} \rtimes H$ be a semidirect product of $\mathbb{R}^{n}$ and a Lie group $H$ which acts smoothly on $\mathbb{R}^{n}$. Let $\eta \in\left(\mathbb{R}^{n}\right)^{*}$ and let $U \subseteq H, \phi: U . \eta \rightarrow \mathbb{R}_{\geq 0}$, and $\mathcal{O}_{\eta} \rightarrow H$, $k \mapsto h_{k}$, as in Lem. 4.14. Let $\tau$ be a representation of $H_{\eta}$ and let $\pi \cong \operatorname{ind}_{G_{\eta}}^{G}\left(\chi_{\eta} \times \tau\right)$ be the representation described in eq. (4.3).

There exists a linear subspace $W \subseteq \mathbb{R}^{n}$ with

$$
\operatorname{dim} W=\operatorname{dim}(U . \eta)=\operatorname{dim}\left(H / H_{\eta}\right)
$$

such that for all $f \in \mathcal{H}_{\pi}$ with $\Theta(f) \in C_{c}\left(U . \eta, \mathcal{H}_{\tau}\right)$

- $w \mapsto\langle\pi(h(w), h) f, g\rangle$ is in $L^{2}(W)$ for almost all $h \in H$ and all $g \in \mathcal{H}_{\pi}$

- and

$$
\int_{W}|\langle\pi(h(w), h) f, g\rangle|^{2} \mathrm{~d} w=\int_{U . \eta} \frac{\left|\left\langle f\left(h_{k}\right), g\left(h h_{k}\right)\right\rangle\right|^{2}}{\phi(k)} \mathrm{d} \nu_{\eta}(k)
$$

Proof. Let $P:\left(\mathbb{R}^{n}\right)^{*} \rightarrow\left(\mathbb{R}^{n}\right)^{*}$ (as in Lem. 4.14) be the projection onto the embedded tangent space $T$. Let

$$
W=\left\{a \in \mathbb{R}^{n} \mid k_{0}(a)=0 \forall k_{0} \in \operatorname{ker}(P)\right\}
$$

Then $\operatorname{dim}(W)=n-\operatorname{dim}(\operatorname{ker}(P))=\operatorname{dim}(T)=\operatorname{dim}(U . \eta)=\operatorname{dim}\left(H / H_{\eta}\right)$.

Recall from eq. 4.5 that the coefficient function of $\pi$ is given by

$$
C_{f} g(h(v), h)=\int_{\mathcal{O}_{\eta}} e^{-2 \pi i k(v)}\left\langle f\left(h_{k}\right), g\left(h h_{k}\right)\right\rangle_{\tau} \mathrm{d} \nu_{\eta}(k) .
$$

By definition of $P$ and $W$ we have $k(w)=P(k)(w)$ for all $w \in W$ and, thus

$$
C_{f} g(h(w), h)=\int_{\mathcal{O}_{\eta}} e^{-2 \pi i P(k)(w)}\left\langle f\left(h_{k}\right), g\left(h h_{k}\right)\right\rangle_{\tau} \mathrm{d} \nu_{\eta}(k) .
$$

Let $F \in C_{c}\left(U . \eta ; \mathcal{H}_{\tau}\right)$ and let $f=\Theta^{-1}(F)$. Using the map $\left.P\right|_{U . \eta}=\Phi: U . \eta \rightarrow U_{T}$, defined in the proof of Lem. 4.14, the coefficient functions can be written as

$$
\begin{aligned}
C_{f} g(h(w), h) & =\int_{U \cdot \eta} e^{-2 \pi i \Phi(k)(w)}\left\langle f\left(h_{k}\right), g\left(h h_{k}\right)\right\rangle_{\tau} \mathrm{d} \nu_{\eta}(k) \\
& =\int_{U_{T}} e^{-2 \pi i t(w)} \frac{\left\langle f\left(h_{\Phi^{-1}(t)}\right), g\left(h h_{\Phi^{-1}(t)}\right)\right\rangle_{\tau}}{\phi\left(\Phi^{-1}(t)\right)} \mathrm{d} \nu_{U_{T}}(t),
\end{aligned}
$$

where $\nu_{U_{T}}$ is the Lebesgue measure on $T$ restricted to $U_{T}$.

By choice of $f$, i.e., $f=\Theta^{-1}(F)$ for $F \in C_{c}\left(U . \eta ; \mathcal{H}_{\tau}\right)$, and since $\phi$ is continuous, the 
function $\frac{|\Theta(f)|^{2}}{\phi}$ on $U . \eta$ is bounded and, therefore,

$$
\begin{aligned}
\int_{U . \eta} \frac{\left|\left\langle f\left(h_{k}\right), g\left(h h_{k}\right)\right\rangle_{\tau}\right|^{2}}{\phi(k)} \mathrm{d} \nu_{\eta}(k) & =\int_{U . \eta} \frac{\left|\left\langle f\left(h_{k}\right), g\left(h h_{k}\right)\right\rangle_{\tau}\right|^{2}}{\phi(k)^{2}} \phi(k) \mathrm{d} \nu_{\eta}(k) \\
& =\int_{U_{T}} \frac{\left|\left\langle f\left(h_{\Phi^{-1}(t)}\right), g\left(h h_{\Phi^{-1}(t)}\right)\right\rangle_{\tau}\right|^{2}}{\phi\left(\Phi^{-1}(t)\right)^{2}} \mathrm{~d} \nu_{U_{T}}(t)
\end{aligned}
$$

is finite. Hence, eq. 4.10) is not only a Fourier transform but also a Plancherel transform. By applying the Plancherel Theorem on eq. 4.11) we get

$$
\int_{W}\left|C_{f} g(h(w), h)\right|^{2} \mathrm{~d} w=\int_{U . \eta} \frac{\left|\left\langle f\left(h_{k}\right), g\left(h h_{k}\right)\right\rangle_{\tau}\right|^{2}}{\phi(k)} \mathrm{d} \nu_{\eta}(k)
$$

for all $g \in \mathcal{H}_{\pi}$.

Now, we are prepared to prove Thm. 4.13

Proof of Thm. 4.13. Let $U$ and $\phi$ be as in Lem. 4.14 and let $W$ be as in Lem. 4.15 Let $\psi_{\tau}$ be an admissible vector for $\tau$. Let $\varphi \in C_{c}(U . \eta)$ nonzero and let $f=\Theta^{-1}\left(\psi_{\tau} \cdot \varphi\right)$. By Lem. 4.15 the function $f$ satisfies

$$
\begin{aligned}
\int_{W}\left|C_{f} g(h(w), h)\right|^{2} \mathrm{~d} w & =\int_{U . \eta} \frac{\left|\left\langle f\left(h_{k}\right), g\left(h h_{k}\right)\right\rangle_{\tau}\right|^{2}}{\phi(k)} \mathrm{d} \nu_{\eta}(k) \\
& =\int_{U . \eta}\left|\left\langle\psi_{\tau}, g\left(h h_{k}\right)\right\rangle_{\tau}\right|^{2} \frac{|\varphi(k)|^{2}}{\phi(k)} \mathrm{d} \nu_{\eta}(k)
\end{aligned}
$$

for all $g \in \mathcal{H}_{\pi}$. By integrating over $H$ we get

$$
\begin{aligned}
\int_{H} \int_{W}\left|C_{f} g(h(w), h)\right|^{2} \mathrm{~d} w \mathrm{~d} h & =\int_{H} \int_{U . \eta}\left|\left\langle\psi_{\tau}, g\left(h h_{k}\right)\right\rangle_{\tau}\right|^{2} \frac{|\varphi(k)|^{2}}{\phi(k)} \mathrm{d} \nu_{\eta}(k) \mathrm{d} h \\
& =\int_{H} \int_{U . \eta}\left|\left\langle\psi_{\tau}, g(h)\right\rangle_{\tau}\right|^{2} \frac{|\varphi(k)|^{2}}{\phi(k) \Delta_{H}\left(h_{k}\right)} \mathrm{d} \nu_{\eta}(k) \mathrm{d} h .
\end{aligned}
$$

Since $\psi_{\tau}$ is admissible, the $H$-integration yields

$$
\begin{aligned}
\int_{H}\left|\left\langle\psi_{\tau}, g(h)\right\rangle_{\tau}\right|^{2} \mathrm{~d} h & =\int_{\mathcal{O}_{\eta}} \int_{H_{\eta}}\left|\left\langle\psi_{\tau}, g\left(h_{k} m\right)\right\rangle_{\tau}\right|^{2} \delta_{H_{\eta}}^{H}(m)^{2} \mathrm{~d} m \mathrm{~d} \nu_{\eta}(k) \\
& =\int_{\mathcal{O}_{\eta}} \int_{H_{\eta}}\left|\left\langle\tau(m) \psi_{\tau}, g\left(h_{k}\right)\right\rangle_{\tau}\right|^{2} \mathrm{~d} m \mathrm{~d} \nu_{\eta}(k) \\
& =\int_{\mathcal{O}_{\eta}}\left\|g\left(h_{k}\right)\right\|_{\tau}^{2} \mathrm{~d} \nu_{\eta}(k) \\
& =\|g\|^{2}
\end{aligned}
$$

By inserting eq. 4.13 in eq. 4.12 we obtain

$$
\int_{H} \int_{W}\left|C_{f} g(h(w), h)\right|^{2} \mathrm{~d} w \mathrm{~d} h=\int_{U . \eta} \frac{|\varphi(k)|^{2}}{\phi(k) \Delta_{H}\left(h_{k}\right)} \mathrm{d} \nu_{\eta}(k) \cdot\|g\|^{2} .
$$


Since $\varphi \in C_{c}(U . \eta)$ and $\varphi \neq 0$, the integral $\int_{U . \eta} \frac{|\varphi(k)|^{2}}{\phi(k) \Delta_{H}\left(h_{k}\right)} \mathrm{d} \nu_{\eta}(k)$ is finite and positive. By normalizing $\varphi$ in a suitable way, we get an admissible function $f=\Theta^{-1}\left(\psi_{\tau} \cdot \varphi\right)$.

\subsubsection{Examples}

To illustrate how Thm. 4.13 can be applied, let us have a look at two examples.

Example 4.16. Let $G=\mathbb{R}^{1+2} \rtimes \mathrm{SO}(1,2)$ and $\eta \in\left(\mathbb{R}^{1+2}\right)^{*}, \eta(v)=\eta_{0} v_{0}$, where $v=$ $\left(v_{0}, v_{1}, v_{2}\right)^{t}$ and $\eta_{0} \neq 0 . \eta$ corresponds to the one-dimensional representation $\chi, \chi(v)=$ $e^{2 \pi i \eta_{0} v_{0}}$. The orbit $\mathcal{O}$ of $\eta$ is the two-sheet hyperboloid consisting of all $k=\left(k_{0}, k_{1}, k_{2}\right)$ satisfying $k_{0}^{2}-k_{1}^{2}-k_{2}^{2}=\left|\eta_{0}\right|^{2}$ and the stabilizer $H_{\eta}$ is given by

$$
H_{\eta}=\left\{\left(\begin{array}{cc}
1 & 0 \\
0 & A_{0}
\end{array}\right) \mid A_{0} \in \mathrm{SO}(2)\right\} .
$$

Since $H_{\eta}$ is compact, all of its irreducible representations are square-integrable. If we choose the trivial representation for $\tau$ then $\pi \cong \operatorname{ind}_{\mathbb{R}^{1+2} \rtimes H_{\eta}}^{\mathbb{R}^{1+2} \rtimes H}(\chi)$ defined on $L^{2}(\mathcal{O})$ has the form

$$
\pi(v, A) f(k)=e^{2 \pi i k(v)} f\left(k A^{-t}\right),
$$

for $v \in \mathbb{R}^{1+2}, A \in S O(1,2), f \in L^{2}(\mathcal{O})$, where $A^{-t}:=\left(A^{-1}\right)^{t}=\left(A^{t}\right)^{-1}$. The tangent space of $\mathcal{O}$ at $\eta$ is given by

$$
\eta+T=\eta+\left\{\left(0, k_{1}, k_{2}\right) \mid k_{1}, k_{2} \in \mathbb{R}\right\}
$$

Let $P:\left(\mathbb{R}^{1+2}\right)^{*} \rightarrow\left(\mathbb{R}^{1+2}\right)^{*}$ be the projection given by

$$
P\left(k_{0}, k_{1}, k_{2}\right)=\left(0, k_{1}, k_{2}\right) \quad \forall k \in\left(\mathbb{R}^{1+2}\right)^{*} .
$$

Every element in $\left(0, k_{1}, k_{2}\right)$ has the two preimages $\left( \pm \sqrt{\left|\eta_{0}\right|^{2}+k_{1}^{2}+k_{2}^{2}}, k_{1}, k_{2}\right)$ in $\mathcal{O}$. By restricting $P$ to the upper sheet of the hyperboloid, i.e., to

$$
U_{\mathcal{O}}=\left\{\left(\sqrt{\left|\eta_{0}\right|^{2}+k_{1}^{2}+k_{2}^{2}}, k_{1}, k_{2}\right) \mid k_{1}, k_{2} \in \mathbb{R}\right\},
$$

we get a diffeomorphism $\Phi:=\left.P\right|_{U_{\mathcal{O}}}$. Since $H$ and $H_{\chi}$ are unimodular, there exists a $H$-invariant measure on $\mathcal{O}$ which restricts to the measure

$$
\mathrm{d} \nu(k)=2 \theta\left(k_{0}\right) \delta_{0}\left(k_{0}^{2}-k_{1}^{2}-k_{2}^{2}-\left|\eta_{0}\right|^{2}\right) \mathrm{d} k_{1} \mathrm{~d} k_{2} \mathrm{~d} k_{3}=\frac{\mathrm{d} k_{1} \mathrm{~d} k_{2}}{\sqrt{\left|\eta_{0}\right|^{2}+k_{1}^{2}+k_{2}^{2}}},
$$

which implies that

$$
\phi(k)=\frac{\mathrm{d} k_{1} \mathrm{~d} k_{2}}{\mathrm{~d} \nu(k)}=\sqrt{\left|\eta_{0}\right|^{2}+k_{1}^{2}+k_{2}^{2}} .
$$


The subspace $W$ is given by

$$
W=\left\{v \in \mathbb{R}^{1+2} \mid \eta(v)=0\right\}=\left\{\left(0, v_{1}, v_{2}\right)^{t} \mid v_{1}, v_{2} \in \mathbb{R}\right\} .
$$

A function $f \in L^{2}\left(U_{\mathcal{O}}\right)$ is admissible if

$$
\int_{U_{\mathcal{O}}} \frac{|f(k)|^{2}}{\phi(k)} \mathrm{d} \nu(k)=1
$$

In that case, we get

$$
\int_{W} \mathrm{~d} w \int_{\mathrm{SO}(1,2)} \mathrm{d} A\left|C_{f} g(A w, A)\right|^{2}=\|g\|^{2}
$$

for all $g \in L^{2}(\mathcal{O})$.

Note that for $\eta=\left(\eta_{0}, \eta_{1}, \eta_{2}\right)$ satisfying $\eta_{0}^{2}-\eta_{1}^{2}-\eta_{2}^{2} \leq 0$ the stabilizer $H_{\eta}$ is isomorphic to $\mathbb{R}$. Thus, no irreducible representation $\tau \in \hat{H}_{\eta}$ is square-integrable. Therefore, Thm. 4.13 cannot be applied.

Although Thm. 4.13 has been developed to study irreducible representations it is not necessary that $\tau$ is irreducible. In particular, the induced representation $\pi=$ $\operatorname{ind}_{\mathbb{R}^{n} \rtimes H_{\eta}}^{G}(\chi \times \tau)$ does not need to be irreducible. All we need is that $\tau$ is squareintegrable. Moreover, it is not necessary that the $H$-orbits in $\left(\mathbb{R}^{n}\right)^{*}$ are locally closed. Therefore, Thm. 4.13 even holds if $G$ is not of type I as the following example shows.

Example 4.17. Let $G=\mathbb{R}^{4} \rtimes_{A} H$ with $H=\mathbb{R}$ and

$$
A(s)=\left(\begin{array}{cccc}
\cos (\gamma s) & -\sin (\gamma s) & & \\
\sin (\gamma s) & \cos (\gamma s) & & \\
& & \cos (s) & -\sin (s) \\
& & \sin (s) & \cos (s)
\end{array}\right)
$$

for a fixed irrational $\gamma \in \mathbb{R} \backslash \mathbb{Q}$. The group $G$ is called the Mautner group and is a classical example of a group which is not of type I. (Details of the Mautner group and its representation theory can be found for example in Baggett [10].)

The orbit of $\eta=(1,0,1,0) \in\left(\mathbb{R}^{4}\right)^{*}$ is dense in the 2-dimensional torus

$$
\{(\cos \alpha, \sin \alpha, \cos \beta, \sin \beta) \mid \alpha, \beta \in \mathbb{R}\} .
$$

The stabilizer $H_{\eta}$ is trivial.

The representation $\pi \cong \operatorname{ind}_{\mathbb{R}^{4}}^{G}\left(e^{2 \pi i \eta}\right)$ is defined on $L^{2}(H)$ and given by

$$
\begin{aligned}
{[\pi(v, s) f](t) } & =e^{2 \pi i \eta(A(-t) v)} f(t-s) \\
& =e^{2 \pi i\left(\cos (\gamma t) v_{1}+\sin (\gamma t) v_{2}+\cos (t) v_{3}+\sin (t) v_{4}\right)} f(t-s)
\end{aligned}
$$

for $f \in L^{2}(H)$ and $v=\left(v_{1}, v_{2}, v_{3}, v_{4}\right)^{t}$. 
The tangent space $\eta+T$ at $\eta$ has the form

$$
\eta+T=\eta+\mathbb{R} \cdot(0, \gamma, 0,1)
$$

We choose

$$
P:\left(\mathbb{R}^{4}\right)^{*} \rightarrow\left(\mathbb{R}^{4}\right)^{*}, \quad\left(k_{1}, k_{2}, k_{3}, k_{4}\right) \mapsto\left(0,0,0, k_{4}\right)
$$

and

$$
W=\left\{\left(0,0,0, v_{4}\right)^{t} \mid v_{4} \in \mathbb{R}\right\} \subseteq \mathbb{R}^{4}
$$

The restriction of $\pi$ to $W \times H$ has the form

$$
[\pi(v, s) f](t)=e^{2 \pi i \sin (t) v_{4}} f(t-s)
$$

for $v=\left(0,0,0, v_{4}\right)^{t}$ which is very similar to what we saw in Ex. 4.11. Indeed, for all $f \in L^{2}(H)$ satisfying

- $\operatorname{supp}(f) \subseteq\left(-\frac{\pi}{2}, \frac{\pi}{2}\right)$

- and

$$
\int_{\mathbb{R}} \frac{|f(t)|^{2}}{|\cos (t)|} \mathrm{d} t=1
$$

we get

$$
\int_{W} \int_{H}\left|C_{f} g(A(s) v, s)\right|^{2} \mathrm{~d} s \mathrm{~d} v=\int_{H} \int_{\mathbb{R}} \frac{|f(t)|^{2}}{|\cos (t)|}|g(t+s)|^{2} \mathrm{~d} t \mathrm{~d} s=\|g\|^{2} .
$$

\subsubsection{Group extensions}

With a few adjustments the results of Thm. 4.13 can be generalized to group extensions. As before, let $H$ be a Lie group which acts smoothly on $\mathbb{R}^{n}$. Furthermore, let

$$
1 \rightarrow \mathbb{R}^{n} \stackrel{\iota}{\rightarrow} G \stackrel{p}{\rightarrow} H \rightarrow 1
$$

be a short exact sequence of Lie groups and let $q: H \rightarrow G$ a fixed Borel cross-section for $p$ with $q\left(e_{H}\right)=e_{G}=\iota(0)$. For every $a \in G$ there exist $v \in \mathbb{R}^{n}$ and $h \in H$ such that $a=\iota(v) q(h)$. To keep the notation simple, we write

$$
a=\iota(v) q(h)=:(v, h) .
$$


Then, multiplication and inversion are given by

$$
\begin{aligned}
\left(v_{1}, h_{1}\right)\left(v_{2}, h_{2}\right) & \left.=\left(v_{1}+h_{1}\left(v_{2}\right)+\omega\left(h_{1}, h_{2}\right)\right), h_{1} h_{2}\right), & & \forall\left(v_{1}, h_{1}\right),\left(v_{2}, h_{2}\right) \in G, \\
(v, h)^{-1} & =\left(-h^{-1} \cdot\left(v-\omega\left(h, h^{-1}\right)\right), h^{-1}\right) & & \\
& \left.=\left(-h^{-1} \cdot v-\omega\left(h^{-1}, h\right)\right), h^{-1}\right), & & \forall(v, h) \in G,
\end{aligned}
$$

where $\omega: H \times H \rightarrow \mathbb{R}^{n}$ is given by

$$
\iota\left(\omega\left(h_{1}, h_{2}\right)\right)=q\left(h_{1}\right) q\left(h_{2}\right) q\left(h_{1} h_{2}\right)^{-1}
$$

The left Haar measure on $G$ still has the form

$$
\int_{G} \varphi(a) \mathrm{d} a=\int_{H} \int_{\mathbb{R}^{n}} \varphi(h . v, h) \mathrm{d} v \mathrm{~d} h, \quad \forall \varphi \in C_{c}(G) .
$$

Again, the action of $H$ on $\mathbb{R}^{n}$ induces actions on $\widehat{\mathbb{R}^{n}}$ and $\left(\mathbb{R}^{n}\right)^{*}$, which are given by

$$
h \cdot \chi_{k}(v)=\chi_{k}\left(h^{-1} \cdot v\right)=e^{2 \pi i k\left(h^{-1} v\right)}=e^{2 \pi i(h . k)(v)},
$$

where we used the notation of eq. (4.2). For a $k \in\left(\mathbb{R}^{n}\right)^{*}$, let $\mathcal{O}_{k}=H . k$ be its orbit and $H_{k}$ its stabilizer. Furthermore, we write $G_{k}=p^{-1}\left(H_{k}\right)$.

In contrast to semidirect products a unitary irreducible representation $\sigma$ of $G_{k}$ which satisfies

$$
\sigma\left(v, e_{H}\right)=\chi_{k}(v) \cdot \mathbb{1}_{\mathcal{H}_{\sigma}}, \quad \forall v \in \mathbb{R}^{n},
$$

can, in general, not be decomposed into a product of $\chi_{k}$ and some irreducible representation of $H_{k}$. Therefore, Mackey's machine for group extensions has a more complicated form (cf. Folland [30], Thm. 6.39 and Thm. 6.40).

Theorem 4.18. Let $G$ be a Lie group extension of a Lie group $H$ by the Lie group $\mathbb{R}^{n}$, i.e.,

$$
1 \rightarrow \mathbb{R}^{n} \rightarrow G \rightarrow H \rightarrow 1
$$

Suppose that the $H$-orbits in $\left(\mathbb{R}^{n}\right)^{*}$ are locally closed.

- If $k \in\left(\mathbb{R}^{n}\right)^{*}$ and $\sigma \in \widehat{G}_{k}$ with $\sigma\left(v, e_{H}\right)=\chi_{k}(v) \cdot \mathbb{1}_{\mathcal{H}_{\sigma}}$, for all $v \in \mathbb{R}^{n}$, then $\operatorname{ind}_{G_{k}}^{G}(\sigma)$ is an irreducible representation of $G$.

- Every irreducible representation of $G$ is of this form.

- Two representations $\operatorname{ind}_{G_{k}}^{G}(\sigma)$ and $\operatorname{ind}_{G_{k^{\prime}}}^{G}\left(\sigma^{\prime}\right)$ are equivalent if and only if $k$ and 
$k^{\prime}$ belong to the same orbit, say $k^{\prime}=m . k$ for some $m \in H$, and

$$
\begin{aligned}
&(v, h) \mapsto \sigma(v, h), \\
&(v, h) \mapsto \sigma^{\prime}\left((0, m)(v, h)(0, m)^{-1}\right),
\end{aligned}
$$

are equivalent representations of $G_{k}=(0, m)^{-1} G_{k^{\prime}}(0, m)$.

Let $\eta \in\left(\mathbb{R}^{n}\right)^{*}$ be fixed and let $\sigma$ be a representation of $G_{\eta}$ with $\sigma\left(v, e_{H}\right)=\chi_{\eta}(v) \cdot \mathbb{1}_{\mathcal{H}_{\sigma}}$, for all $v \in \mathbb{R}^{n}$. Let

$$
\tau: H \rightarrow \mathcal{U}\left(\mathcal{H}_{\sigma}\right), \quad \tau(h)=\sigma(0, h) .
$$

As mentioned above, $\tau$ is, in general, not a representation. Indeed, it is a projective representation of $H_{\eta}$. That means that, together with the function

$$
\chi_{\eta} \circ \omega: H_{\eta} \times H_{\eta} \rightarrow U(1),
$$

$\tau$ satisfies

$$
\begin{aligned}
\tau\left(m_{1}\right) \tau\left(m_{2}\right) & =\chi_{\eta}\left(\omega\left(m_{1}, m_{2}\right)\right) \cdot \tau\left(m_{1} m_{2}\right), & & \forall m_{1}, m_{2} \in H_{\eta}, \\
\tau\left(m^{-1}\right) & =\chi_{\eta}\left(\omega\left(m^{-1}, m\right)\right) \cdot \tau(m)^{*}, & & \forall m \in H_{\eta} .
\end{aligned}
$$

Let $v_{1}, v_{2} \in \mathcal{H}_{\sigma}$ be arbitrary vectors. The coefficient functions of $\sigma$ are given by

$$
(v, m) \mapsto C_{v_{1}} v_{2}(v, m)=\left\langle\sigma(v, m) v_{1}, v_{2}\right\rangle=\chi_{\eta}(-v)\left\langle\tau(m) v_{1}, v_{2}\right\rangle
$$

for all $v \in \mathbb{R}^{n}, m \in H_{\eta}$. Since the modulus of $C_{v_{1}} v_{2}$,

$$
\left|C_{v_{1}} v_{2}(v, m)\right|^{2}=\left|\left\langle\tau(m) v_{1}, v_{2}\right\rangle\right|^{2},
$$

does not depend on $v \in \mathbb{R}^{n}, \sigma$ is said to be square-integrable modulo $\mathbb{R}^{n}$ (in the sense of Perelomov as discussed in Chp. 3.1.3 if there exists a vector $\psi_{\tau} \in \mathcal{H}_{\sigma}$ such that

$$
\int_{H_{\eta}}\left|C_{\psi_{\tau}} v(m)\right|^{2} \mathrm{~d} m=\|v\|^{2}, \quad \forall v \in \mathcal{H}_{\sigma}
$$

Recall that $\mathcal{O}_{\eta}$ is endowed with the quotient topology, which does not necessarily coincide with the subspace topology. For a given regular Borel section

$$
\mathcal{O}_{\eta} \rightarrow H, \quad k \mapsto h_{k},
$$

let $\nu_{\eta}$ be the associated quasi-invariant Radon measure on $\mathcal{O}_{\eta}$ (cf. Lem. 2.33). The representation $\pi \cong \operatorname{ind}_{G_{\eta}}^{G}(\sigma)$ can be realized on the Hilbert space $\mathcal{H}_{\pi}=L^{2}\left(G, G_{\eta}, \delta_{H_{\eta}}^{H} \tau\right)$ 
given by

$$
\begin{array}{rlrl}
f(v, h) & =e^{-2 \pi i h . \eta(v)} f(0, h) & & \forall v \in \mathbb{R}^{n}, h \in H, \\
f(0, h m) & =\delta_{H_{\eta}}^{H}\left(m^{-1}\right) e^{2 \pi i h . \eta(\omega(h, m))} \tau(m)^{*} f(0, h) & \\
& =\delta_{H_{\eta}}^{H}\left(m^{-1}\right) e^{2 \pi i h . \eta\left(\omega(h, m)-\omega\left(h, h^{-1}\right)\right)} \tau\left(m^{-1}\right) f(0, h), & & \\
\langle f, g\rangle & =\int_{\mathcal{O}_{\eta}}\left\langle f\left(0, h_{k}\right), g\left(0, h_{k}\right)\right\rangle_{\tau} \mathrm{d} \nu_{\eta}(k), &
\end{array}
$$

and has the form

$$
\begin{aligned}
{\left[\pi\left(v, e_{H}\right) f\right]\left(h^{\prime}\right) } & =e^{2 \pi i\left(h^{\prime} \cdot \eta\right)(v)} f\left(h^{\prime}\right), \\
{[\pi(0, h) f]\left(h^{\prime}\right) } & =e^{2 \pi i h^{\prime} \cdot \eta\left(\omega\left(h, h^{-1} h^{\prime}\right)\right)} f\left(h^{-1} h^{\prime}\right) .
\end{aligned}
$$

The coefficient functions of $\pi$ are given by

$$
\begin{aligned}
C_{f} g(h(v), h) & =\langle\pi(h(v), h) f, g\rangle=\left\langle\pi\left(v, e_{H}\right) f, \pi(0, h)^{*} g\right\rangle \\
& =\int_{\mathcal{O}_{\eta}} e^{-2 \pi i k(v)}\left\langle f\left(h_{k}\right),\left[\pi(0, h)^{*} g\right]\left(h_{k}\right)\right\rangle_{\tau} \mathrm{d} \nu_{\eta}(k) \\
& =\int_{\mathcal{O}_{\eta}} e^{-2 \pi i k\left(v+h^{-1} \cdot \omega\left(h, h_{k}\right)\right)}\left\langle f\left(h_{k}\right), g\left(h h_{k}\right)\right\rangle_{\tau} \mathrm{d} \nu_{\eta}(k) .
\end{aligned}
$$

Theorem 4.19. Let $G$ be a Lie group extension of a Lie group $H$ by the Lie group $\mathbb{R}^{n}$. Let $\eta \in\left(\mathbb{R}^{n}\right)^{*}$ and let $\sigma$ be a representation of $G_{\eta}$ which satisfies $\sigma\left(v, e_{H}\right)=\chi_{k}(v) \cdot \mathbb{1}_{\mathcal{H}_{\sigma}}$, for all $v \in \mathbb{R}^{n}$, and is square-integrable modulo $\mathbb{R}^{n}$.

Then there exists a Radon measure $\mu$ on $G$ such that $\pi=\operatorname{ind}_{G_{\eta}}^{G}(\sigma)$ is $\mu$-squareintegrable. $\mu$ has the form

$$
\int_{G} \varphi(a) \mathrm{d} \mu(a)=\int_{H} \int_{W} \varphi(h(w), h) \mathrm{d} w \mathrm{~d} h, \quad \forall \varphi \in C_{c}(G),
$$

where $W \subseteq \mathbb{R}^{n}$ is a linear subspace of dimension $\operatorname{dim}(W)=\operatorname{dim}\left(H / H_{\eta}\right)$.

The proof follows analogously to what we saw before. Lem. 4.14 applies unchanged. In the proof of Lem. 4.15 one has to add the unitary factor $e^{-2 \pi i k\left(h^{-1} \cdot \omega\left(h, h_{k}\right)\right)}$ in terms for the coefficient function. Using the same argument as above leads to the equation

$$
\begin{aligned}
\int_{W}\left|C_{f} g(h(w), h)\right|^{2} \mathrm{~d} w & =\int_{U . \eta} \frac{\left|e^{-2 \pi i k\left(h^{-1} \cdot \omega\left(h, h_{k}\right)\right)} \cdot\left\langle f\left(h_{k}\right), g\left(h h_{k}\right)\right\rangle_{\tau}\right|^{2}}{\phi(k)} \mathrm{d} \nu_{\eta}(k) \\
& =\int_{U . \eta} \frac{\left|\left\langle f\left(h_{k}\right), g\left(h h_{k}\right)\right\rangle_{\tau}\right|^{2}}{\phi(k)} \mathrm{d} \nu_{\eta}(k),
\end{aligned}
$$

for all $\Theta(f) \in C_{c}\left(U . \eta ; \mathcal{H}_{\sigma}\right)$ and $g \in \mathcal{H}_{\pi}$. With the updated notation the proof of Thm. 4.13 remains unchanged. 


\subsection{Continuous wavelet transformations on manifolds}

In [6], Antoine and Vandergheynst present a group-theoretical approach to define the continuous wavelet transformation on the 2 -sphere $M=\mathbb{S}^{2}$ as a homogeneous space of $\mathrm{SO}(3)$. As described in Chp. 3.1.5 they construct a group $G$ such that

(i) $G$ acts transitively on $\mathbb{S}^{2}$,

(ii) $G$ acts locally by translations, rotations, and dilations (cf. Def. 4.20 below),

(iii) and the corresponding unitary representation of $G$ in $L^{2}\left(\mathbb{S}^{2}\right)$ is square-integrable (in a generalized sense) and defines a continuous wavelet transformation on the sphere.

The second assumption is motivated by the article [40] of Holschneider. The idea is that continuous wavelet transformations on manifolds should be similar to the classic continuous wavelet transformations and its generalization to $\mathbb{R}^{n}$ as a homogeneous space of the similitude group $\operatorname{SIM}(n)$ as discussed in Chp. 3.1.1 and Chp. 3.1.2. We want to address the question whether this procedure can be used to construct a continuous wavelet transformation on manifolds other than $\mathbb{R}^{n}$ and $\mathbb{S}^{n}$.

Let $M$ be a connected manifold of dimension $n$ endowed with a smooth transitive action

$$
\gamma: G \rightarrow \operatorname{Diffeo}(M)
$$

of a connected Lie group $G$, where $\operatorname{Diffeo}(M)$ is the group of diffeomorphisms $M \rightarrow M$. For some point $x \in M$, the stabilizer $G_{x}$ of $x$ acts linearly on the tangent space $T_{x} M$ by

$$
T_{x} \gamma(b): T_{x} M \rightarrow T_{x} M, \quad \text { for } b \in G_{x},
$$

where $T_{x} \gamma(b)$ is the tangent map (pushforward) of $\gamma(b): M \rightarrow M$ at $x$.

Definition 4.20. We say that the stabilizer $G_{x}$ acts locally by rotations and dilations if there is an isomorphism $\psi: T_{x} M \rightarrow \mathbb{R}^{n}$ such that

$$
\left\{\psi \circ T_{x} \gamma(b) \circ \psi^{-1} \mid b \in G_{x}\right\}=\mathrm{SO}(n) \cdot \mathbb{R}_{>0} \subseteq \mathrm{Gl}(n) .
$$

In that case, we say that $G$ acts locally by translations, rotations, and dilations on $M$.

In [5], Antoine and Vandergheynst pointed out that if $M$ has a Riemannian metric $\eta$, then (a closed subgroup of) the group of conformal transformations $\operatorname{Conf}(M, \eta)$ is the right choice for $G$. In fact, in [6] they used the conformal group $G=\mathrm{SO}_{0}(3,1)$ of $\mathbb{S}^{2}$. However, the assumption that $G$ acts by translations, rotations, and dilations already implies that there is a $G$-conformal metric $\eta$ on $M$ as the following lemma shows. 
Lemma 4.21. Let $M$ be a connected manifold of dimension $n \geq 2$. Suppose that there is a Lie group $G$ such that

(i) there is a smooth transitive action $\gamma: G \rightarrow \operatorname{Diffeo}(M)$

(ii) and the stabilizer $G_{x}$ of some $x \in M$ acts locally by rotations and dilations.

Then there is a metric $\eta$ on $M$ such that $\gamma(G) \subseteq \operatorname{Conf}(M, \eta)$.

Proof. In the following $x$ is a fixed element of $M$ and we write $H:=G_{x}$. Furthermore, the action of $G$ on $M$ is denoted by

$$
a . y:=\gamma_{y}(a):=\gamma(a)(y)
$$

for $a \in G, y \in M$.

With use of $\psi$ of Def. 4.20 , we can define a scalar product $\nu \in T^{2}\left(T_{x} M\right):=\left(T_{x} M\right)^{*} \otimes$ $\left(T_{x} M\right)^{*}$ on the tangent space of $x$ by

$$
\nu(X, Y)=\langle\psi(X), \psi(Y)\rangle_{\mathrm{eucl}}, \quad \forall X, Y \in T_{x} M,
$$

where $\langle\cdot, \cdot\rangle_{\text {eucl }}$ is the Euclidean scalar product on $\mathbb{R}^{n}$. For $a \in G$, let

$$
a . \nu:=\gamma\left(a^{-1}\right)^{*} \nu \in T^{2}\left(T_{a . x} M\right)
$$

be the pullback scalar product. The stabilizer $H$ acts on $\nu$ by

$$
\text { b. } \nu=\gamma\left(b^{-1}\right)^{*} \nu=\operatorname{det}\left(T_{x} \gamma(b)\right)^{-2 / n} \nu, \quad \forall b \in H .
$$

In particular, by eq. 4.14 we have

$$
(a H) . \nu=\mathbb{R}_{>0} \cdot a . \nu, \quad \forall a \in G
$$

and $\nu$ does not extend to a $G$-invariant metric on $M$.

Since $\gamma_{x}: G \rightarrow M$ is a principal $H$-bundle, there exists an open covering of $M$ by local trivializations $\left\{\varphi_{i}: U_{i} \times H \rightarrow \gamma_{x}^{-1}\left(U_{i}\right)\right\}_{i \in I}$. Let $\left\{\chi_{i}\right\}_{i \in I}$ a partition of unity subordinate to $\left\{U_{i}\right\}_{i \in I}$ and define $\eta \in T^{2} M:=T^{*} M \otimes T^{*} M$ by

$$
\eta_{y}=\sum_{i \in I: y \in U_{i}} \chi_{i}(y) \cdot\left(\varphi_{i}(y, e) . \nu\right) \in T^{2}\left(T_{y} M\right), \quad \forall y \in M
$$

where $e \in H \subseteq G$ is the identity element. By construction $\eta$ is smooth and positive definite. Hence, it defines a Riemannian metric on $M$.

It remains to show that $G$ acts conformally on $(M, \eta)$. Let $a \in G$. By eq. 4.15), for every $y \in M$ there exists a positive constant $c_{a}(y)>0$ such that

$$
a . \eta_{y}=c_{a}(y) \cdot \eta_{a . y} .
$$


Let $X$ be a smooth vector field which is nonzero on a neighborhood $U$ of $y \in M$. Then, the function $c_{a}: M \rightarrow \mathbb{R}_{>0}$ satisfies

$$
c_{a}(z)=\frac{\left(a . \eta_{z}\right)\left(X_{a . z}, X_{a . z}\right)}{\eta_{a . z}\left(X_{a . z}, X_{a . z}\right)}, \quad \forall z \in a . U .
$$

Thus, $\left.c_{a}\right|_{a . U}$ is smooth because the right hand side of eq. 4.16 is. It follows that $c_{a}$ is smooth.

Note that $\eta$ is unique up to conformal equivalence. This is because, (due to eq. (4.14)) $\nu \in T^{2}\left(T_{x} M\right)$ is unique up to multiplication by a positive constant. Therefore, $\eta_{x}$ and, by transitivity of $G, \eta_{y}$ for all $y \in M$ is unique up to a positive factor. Moreover, by eq. 4.15 the action of $G$ on $(M, \eta)$ is essential. That is, for every metric $\eta^{\prime}$ which is conformally equivalent to $\eta$, there are $a \in G$ such that $\gamma(a)$ is not an isometry of $\left(M, \eta^{\prime}\right)$. This has deep consequences as the following theorem shows, which is is known as Riemannian Lichnerowicz conjecture (cf. Alekseevskii and Baum [2]) and has been proven by Ferrand [29] in 1996.

Theorem 4.22. Let $(M, \eta)$ be a connected Riemannian manifold of dimension $n \geq 2$. If the conformal group $\operatorname{Conf}(M, \eta)$ is essential, then $(M, \eta)$ is conformally diffeomorphic

- to $\left(\mathbb{S}^{n}, \eta_{\text {can }}\right)$ if $M$ is compact,

- or to $\left(\mathbb{R}^{n}, \eta_{\text {eucl }}\right)$ if $M$ is not compact,

where $\eta_{\text {can }}$ is the canonical metric on $\mathbb{S}^{n}$ and $\eta_{\mathrm{eucl}}$ is the Euclidean metric on $\mathbb{R}^{n}$.

As a consequences, we get the following result.

Corollary 4.23. Let $M$ be a connected manifold of dimension $n \geq 2$. Suppose that there is a Lie group $G$ such that

(i) $G$ acts smoothly and transitively on $M$

(ii) and the stabilizer $G_{x}$ of some $x \in M$ acts locally by rotations and dilations.

Then $M$ is diffeomorphic to $\mathbb{S}^{n}$ or $\mathbb{R}^{n}$.

Cor. 4.23 shows that (except for $\mathbb{R}^{n}$ ) the $n$-sphere $\mathbb{S}^{n}$ is the only manifold for which the construction of Antoine and Vandergheynst can be done. Hence, in order to construct a continuous wavelet transformation for other homogeneous manifolds it is necessary to go away from the classical continuous wavelet transformation and consider more general concepts. 


\section{CHAPTER 5}

\section{Outlook and discussion}

As shown by Führ in [33] and seen in Chp. 3.2, the relationship between a given unitary representation $\pi$ of type I of a second countable locally compact group $G$ and its the left regular representation $\lambda_{G}$ is crucial for square-integrability and the existence of admissible vectors. $\pi$ is square-integrable only if it is a subrepresentation of $\lambda_{G}$. To decide whether the representation $\pi$ is a subrepresentation of $\lambda_{G}$, it is essential to have good knowledge of the Plancherel decomposition of $\lambda_{G}$ and its Plancherel measure $\mu_{G}$ as well as the central decomposition of $\pi$ and its central measure $\mu_{\pi}$. As seen in Chp. 3.2.2 $\pi$ is a subrepresentation of $\lambda_{G}$ if and only if

- $\mu_{\pi}$ is $\mu_{G}$-absolutely continuous;

- and the multiplicities $m_{\pi}(\sigma)$ of $\mu_{G}$-almost all irreducible representations $\sigma$ appearing in the central decomposition of $\pi$ satisfy $m_{\pi}(\sigma) \leq \operatorname{dim}(\sigma)$.

If $G$ is nonunimodular, then $\mu_{G}$-almost all irreducible representations of $G$ are infinitedimensional and it turns out that $\pi$ is square-integrable if and only if $\mu_{\pi}$ is $\mu_{G}$-absolutely continuous (cf. Thm. 3.16). If $G$ is unimodular, then the situation is more complicated. On the one hand, the set of finite-dimensional irreducible representations of $G$ is not necessarily $\mu_{G}$-negligible. On the other hand, being a subrepresentation of $\lambda_{G}$ is not sufficient. Another condition is needed, namely, $\pi$ has to satisfy

$$
\int_{\hat{G}} m_{\pi}(\sigma) \mathrm{d} \mu_{G}<\infty .
$$

Hence, there are basically two reasons why the representation $\pi$ is not square-integrable. The first one is that it is "too small" in the sense that $\mu_{\pi}$ and $\mu_{G}$ are mutually singular. This problem occurs for both classes of groups. The second one is that $\pi$ is "too large" in the sense that $\mu_{\pi}$ is $\mu_{G}$-absolutely continuous, however, the multiplicities $\mu_{\pi}(\sigma)$ (of some) of the irreducible representations $\sigma$ are "too large". This situation only occurs for unimodular groups.

In general, it is possible that $\mu_{\pi}$ is neither $\mu_{G}$-singular nor $\mu_{G}$-absolutely continuous. As shown in Chp. 4.1. the Lebesgue decomposition theorem yields a decomposition $\mu_{\pi}=\nu_{\ll}+\nu_{\perp}$ into a measure $\nu_{\ll}$ which is $\mu_{G}$-absolutely continuous and a measure $\nu_{\perp}$ which is $\mu_{G}$-singular. This decomposition gives rise to a decomposition $\pi=\pi_{\ll} \oplus \pi_{\perp}$, where $\pi_{\perp}$ is a "small" (i.e., disjoint to $\lambda_{G}$ ) and $\pi_{\ll}$ is a "large" representation (i.e., 
quasi-equivalent to a subrepresentation of $\lambda_{G}$ ) of $G$ (cf. Thm. 4.2). Hence, we can study both problems separately.

If $\pi$ is a representation which is "too large", then breaking it down into smaller representations seems to be a successful strategy. In Chp. 4.2 it is shown that such a representation can be decomposed into a countable direct sum $\pi=\bigoplus_{i \in I} \pi_{i}$ of squareintegrable subrepresentations $\pi_{i}$. Then, by fixing an admissible vector $\psi_{i}$ for each $\pi_{i}$, one can verify that every $f \in \mathcal{H}_{\pi}$ satisfies

$$
\sum_{i \in I} \int_{G}\left|\left\langle\pi_{i}(a) \psi_{i}, f\right\rangle\right|^{2} \mathrm{~d} a=\|f\|^{2}
$$

and the operator $W: \mathcal{H}_{\pi} \rightarrow L^{2}(G \times I)$

$$
W f(a, i)=\left\langle\pi_{i}(a) \psi_{i}, f\right\rangle
$$

defines a generalized continuous wavelet transformation (cf. Cor. 4.8). The decomposition $\pi=\bigoplus_{i \in I} \pi_{i}$ is essentially arbitrary and can be chosen correspondingly. As mentioned above, this procedure is only necessary for unimodular groups.

The strategy of enlarging the domain of integration from $G$ to larger spaces is not new. Ebert and Wirth [26], Führ [35] and Antoine and Vandergheynst [6] follow different approaches to solve the same kind of problem and end up with different generalized continuous wavelet transformations with values in $L^{2}\left(G \times \mathbb{R}_{>0}\right)$ (cf. Chp. 3.1.5 and Chp. 3.1.6). In fact, the strategy of Ebert and Wirth led to the idea presented in Chp. 4.2 .

By Thm. 2.65, "large" representations are quasi-equivalent to the left regular representation. There exists a smallest cardinal number $d \in \mathbb{N} \cup\{\infty\}$ such that $\pi$ is a subrepresentation of $d \cdot \lambda$. If $d=1$ then $\pi$ is cyclic. If $d \geq 1$ then $\pi$ can be decomposed into a direct sum of $d$ cyclic representations. From Thm. 2.66, one can deduce that every cyclic subrepresentation of a "large" representation is a subrepresentation of $\lambda_{G}$. It follows that the cardinality of $I$ is bounded from below by $d$. If ineq. (5.1) is satisfied, then each of the $d$ cyclic representations satisfies the corresponding analogue of ineq. (5.1) and, therefore, they are square-integrable. Hence, there exists a decomposition $\pi=\bigoplus_{i \in I} \pi_{i}$ into square-integrable subrepresentations with $|I|=d$. However, if $\pi=\bigoplus_{j \in J} \pi_{j}$ is a finite decomposition into cyclic subrepresentations and $\pi$ does not satisfy ineq. (5.1), then at least one of the representations $\pi_{j}$ does not satisfy the corresponding analogue of ineq. (5.1). Hence, we have $|I|=\infty$ if ineq. (5.1) is not fulfilled.

For a moment, let us consider the problem from a different point of view. Let $\pi$ be a unitary representation and suppose that there exists an isometry $V: \mathcal{H}_{\pi} \rightarrow L^{2}(G \times M)$ for some measure space $\left(M, \mu_{M}\right)$ such that

$$
[V \pi(b) f](a, m)=V f\left(b^{-1} a, m\right), \quad \forall f \in \mathcal{H}_{\pi} .
$$


Then, for all $\varphi \in L^{2}(M)$, the operator $V^{\varphi}: \mathcal{H}_{\pi} \rightarrow L^{2}(G)$,

$$
V^{\varphi} f(a)=\int_{M} V f(a, m) \cdot \varphi(m) \mathrm{d} \mu_{M}(m),
$$

defines an intertwiner between $\pi$ and $\lambda_{G}$. Since $V$ is injective, there exists at least one $\varphi \in L^{2}(M)$ such that $V^{\varphi}$ is nonzero. Hence, $\pi$ and $\lambda_{G}$ are not disjoint. It follows that the approach of enlarging the domain of integration does only work for "large" representations.

In reverse, if $\pi$ is a "small" representation, i.e., disjoint from $\lambda_{G}$, then the approach of enlarging the domain of integration has no chance to work. It turns out that this situation is more challenging. Instead of enlarging the domain of integration, one can try to reduce it. In fact, this is exactly what Perelomov and de Bièvre do in the approaches discussed in Chp. 3.1.3 and Chp. 3.1.4. However, in order to find a suitable domain it is important to have good knowledge of the dual of $G$. Since the structure of the dual can be very complicated, this is a very hard task. In Chp. 4.3 , we study semidirect products

$$
G=\mathbb{R}^{n} \rtimes H
$$

and group extensions

$$
1 \rightarrow \mathbb{R}^{n} \rightarrow G \rightarrow H \rightarrow 1
$$

of the Lie groups $\mathbb{R}^{n}$ and $H$. Those groups have usually fairly easy duals, which is wellknown due to Mackey's machine (cf. Thm. 4.9 and Thm. 4.18). Starting from these results, one can show that for a certain class of representations $\pi$ it is possible to find a Radon measure $\mu$ on $G$ such that $\pi$ is $\mu$-square-integrable (cf. Thm. 4.13 and Thm.4.19). That is, there exists a vector $\psi \in \mathcal{H}_{\pi}$ such that the operator $W_{\psi}: \mathcal{H}_{\pi} \rightarrow L^{2}(G, \mu)$,

$$
W_{\psi} f(a)=\langle\pi(a) \psi, f\rangle, \quad \forall f \in \mathcal{H}_{\pi},
$$

is an isometry, i.e.,

$$
\int_{G}\left|W_{\psi} f(a)\right|^{2} \mathrm{~d} \mu(a)=\|f\|^{2} .
$$

Similarly to the construction by de Bièvre in [20], this result is not covered by the considerations of Perelomov in [55] and extends the theory. Indeed, the ideas presented by de Bièvre were the starting point for the construction in Chp. 4.3. In [20] the author studies irreducible representations of semidirect products $G=\mathbb{R}^{n} \rtimes H$ using the connection between irreducible representations and coadjoint orbits as well as the symplectic structure of coadjoint orbits. In the end, de Bièvre gives an admissibility criterion which is not very explicit. In contrast, the construction presented in this thesis 
uses a different strategy. It turns out that this approach works not only for irreducible representations of semidirect products but also for certain reducible representations of group extensions. Moreover, an explicit sufficient criterion is given in Thm. 4.13 and Thm. 4.19,

There is some hope that the results of Chp. 4.3 can be generalized to all irreducible representations of nilpotent Lie groups. On the one hand, the structure of the dual of nilpotent Lie groups is well-known (see for instance Brown [17, Kirillov [46], Lipsman and Rosenberg [49]). On the other hand, there exists a series of subgroups

$$
1=Z_{0} \subset Z_{1} \subset \cdots Z_{n-1} \subset Z_{n}=G
$$

such that $Z_{i} \unlhd G$ and $Z_{i} / Z_{i-1} \cong \mathbb{R}^{n_{i}}$. It might be possible to successively construct a generalized continuous wavelet transformation on $G / Z_{i-1}$ using the generalized continuous wavelet transformation on $G / Z_{i}$, since the latter is a Lie group extension of the form

$$
1 \rightarrow Z_{i} / Z_{i-1} \rightarrow G / Z_{i-1} \rightarrow \underbrace{\left(G / Z_{i-1}\right) /\left(Z_{i} / Z_{i-1}\right)}_{\cong G / Z_{i}} \rightarrow 1
$$

However, the generalization to nilpotent Lie groups is not straightforward and it is necessary to put some effort in it. 


\section{APPENDIX A}

\section{Polar decomposition of direct integral operators}

Lemma A.1. Let $(A, \mu)$ be a measure space and let $\left\{\mathcal{H}_{a}\right\}_{a \in A}$ be a measurable field of separable Hilbert spaces.

- Let $\{S(a)\}_{a \in A}$ be a measurable field of operators which is almost everywhere bounded and positive. Then $\left\{S(a)^{\frac{1}{2}}\right\}_{a \in A}$ is measurable.

- Let $\{T(a)\}_{a \in A}$ be a measurable field of operators which is almost everywhere bounded and let $T(a)=U(a)|T(a)|$ be the polar decomposition of $T(a)$. Then $\{U(a)\}_{a \in A}$ and $\{|T(a)|\}_{a \in A}$ are measurable.

Let $\mathcal{H}$ be a (complex) Hilbert space and denote the space of bounded operators on $\mathcal{H}$ by $\mathcal{B}(\mathcal{H})$. It is well-known that any bounded operator $A \in \mathcal{B}(\mathcal{H})$ can be uniquely written as a product

$$
A=U|A|,
$$

where $U \in \mathcal{B}(\mathcal{H})$ is a partial isometry on $\overline{\operatorname{im}(A)}$, i.e., $U^{*} U$ is the orthogonal projection onto $\overline{\operatorname{im}(A)}$, and $|A|=\left(A^{*} A\right)^{\frac{1}{2}} \in \mathcal{B}(\mathcal{H})$ is defined via functional calculus. The decomposition in eq. A.1 is called the polar decomposition of $A$.

In this chapter, we give a concrete construction of the polar decomposition. To begin with, recall that the square root of a non-negative number $s$ can be iteratively computed by the Babylonian method. Let $f: \mathbb{R}_{>0} \rightarrow \mathbb{R}_{>0}$ be the function

$$
f(x)=\frac{1}{2}\left(x+x^{-1} s\right) .
$$

and define the sequence $\left(b_{n}\right)_{n \in \mathbb{N}_{0}}$ by choosing some $b_{0}>0$ and computing $b_{k+1}=f\left(b_{k}\right)$. Note that $b_{n} \geq \sqrt{s}$ for all $n \geq 1$ as

$$
f(x)-\sqrt{s}=\frac{(x-\sqrt{s})^{2}}{2 x} \geq 0 .
$$

W.l.o.g. we can assume that $b_{0} \geq \sqrt{s}$ (by shifting the indices by 1 ) and restrict $f$ to a function $f:[\sqrt{s}, \infty) \rightarrow[\sqrt{s}, \infty)$. Then $f$ is a contraction as $\left|f^{\prime}(x)\right| \leq \frac{1}{2}$ for all $x \geq \sqrt{s}$ and therefore by the Banach Fixed-Point Theorem $\left(b_{n}\right)_{n \in \mathbb{N}_{0}}$ converges to the unique 
solution of $x=f(x)$ in the domain of $f$, namely $x=\sqrt{s}$. In particular, for all $x \leq b_{0}$

$$
0 \leq \underbrace{f(\cdots f(x) \cdots)}_{n \text { times }}-\sqrt{s} \leq 2^{-n}(x-\sqrt{s}) \leq 2^{-n} b_{0}
$$

and the interval $\left[\sqrt{s}, b_{0}\right]$ is mapped to itself.

Instead of a positive real number $s$, we want to apply this method to a positive operator $S \in \mathcal{B}(\mathcal{H})$. Let $b \geq\|S\|^{\frac{1}{2}}, b>0$, and note that then the spectrum $\sigma(S)$ of $S$ is contained in $\left[0, b^{2}\right]$. Define $F: \mathbb{R}_{>0} \times\left[0, b^{2}\right] \rightarrow \mathbb{R}_{>0}$ by

$$
F(x, s)=\frac{1}{2}\left(x+x^{-1} s\right) .
$$

and let $\left(b_{n}\right)_{n \in \mathbb{N}_{0}}$ be the sequence of functions $\left[0, b^{2}\right] \rightarrow \mathbb{R}_{>0}$ defined by

$$
b_{0}(s)=b, \quad b_{k+1}(s)=F\left(b_{k}(s), s\right) .
$$

From eq. A.2 and since $\left.F(\cdot, s)\right|_{[\sqrt{s}, \infty)}$ is a contraction, it follows that

$$
\sqrt{s} \leq F(x, s) \leq b \quad \forall x \in[\sqrt{s}, b] .
$$

Consequently, the functions $b_{n}$ satisfy

$$
\sqrt{s} \leq b_{n}(s) \leq b \quad \forall n \in \mathbb{N}_{0} .
$$

Moreover, $F(x, s) \geq \frac{1}{2} x$ and therefore

$$
\operatorname{im}\left(b_{n}\right) \subseteq\left[2^{-n} b, b\right] \quad \forall n \in \mathbb{N}_{0} .
$$

Hence, the operators $B_{n}=b_{n}(S)$ are bounded and invertible as $\sigma\left(B_{n}\right) \subseteq\left[2^{-n} b, b\right]$.

By ineq. A.3 the sequence of functions $\left(b_{n}\right)_{n \in \mathbb{N}_{0}}$ converges uniformly to the function $[0, b] \rightarrow \mathbb{R}_{>0}, s \mapsto \sqrt{s}$. Thus, the sequence $\left(B_{n}\right)_{n \in \mathbb{N}_{0}}$ converges to $S^{\frac{1}{2}}$ in the norm topology.

Now, let $T \in \mathcal{B}(\mathcal{H})$. Let $b \geq\|T\|, b>0$ and $B_{n}=b_{n}\left(T^{*} T\right)$ for $n \in \mathbb{N}_{0}$, where $\left(b_{n}\right)_{n \in \mathbb{N}_{0}}$ is the sequence functions defined in eq. A.4. Recall that all $B_{n}$ are invertible and converge to $|T|$. Define the sequence $\left(U_{n}\right)_{n \in \mathbb{N}_{0}}$ in $\mathcal{B}(\mathcal{H})$ by

$$
U_{n}=T B_{n}^{-1}
$$

Then

$$
\left\|U_{n}\right\|=\left\||T| B_{n}^{-1}\right\| \leq 1
$$

because $0 \leq \frac{\sqrt{s}}{b_{n}(s)} \leq 1$ for all $n \in \mathbb{N}_{0}$. Since the unit ball in $\mathcal{B}(\mathcal{H})$ is ultraweakly compact the sequence $\left(U_{n}\right)_{n \in \mathbb{N}_{0}}$ has a ultraweakly convergent subsequence. Denote its limit by 
$U$ and note that $\|U\| \leq 1$ as the norm is ultraweakly lower semi-continuous. Moreover, $U_{n}|T|$ converges (in the norm topology) to $T$ as

$$
\left\|T-T B_{n}^{-1}|T|\right\|=\left\||T|-|T| B_{n}^{-1}|T|\right\| \leq 2^{-n} b
$$

because $0 \leq \sqrt{s}-\frac{s}{b_{n}(s)}=\frac{\sqrt{s}}{b_{n}(s)}\left(b_{n}(s)-\sqrt{s}\right) \leq 1 \cdot 2^{-n} b$. On the one hand, for all $f \in \operatorname{im}(|T|)$, say $f=|T| g$, we have

$$
U f=T g
$$

and in consequence $U$ uniquely defined on $\operatorname{im}(|T|)$ and by continuity it is also uniquely defined on $\overline{\operatorname{im}(|T|)}$. On the other hand, let $f \in \operatorname{im}(|T|)^{\perp}$. Since $|T|$ is self-adjoint it follows that $f \in \operatorname{ker}(|T|)$ and therefore

$$
\left\|U_{n} f\right\|=\left\|T B_{n}^{-1} f\right\|=\left\||T| B_{n}^{-1} f\right\|=\left\|B_{n}^{-1}|T| f\right\|=0 .
$$

Hence, $U$ is uniquely defined on $\operatorname{im}(|T|)^{\perp}$. By uniqueness of $U$ it follows that $\left(U_{n}\right)_{n \in \mathbb{N}_{0}}$ converges ultraweakly to $U$ (and not only a subsequence). In particular, $\left(U_{n}\right)_{n \in \mathbb{N}_{0}}$ converges weakly to $U$.

Proof of Lem. A.1. Let $T=\{T(a)\}_{a \in A}$ be a measurable field of operators which is almost everywhere bounded and positive. Define

$$
\begin{aligned}
B_{n}(a) & :=b_{n}\left(T(a)^{*} T(a)\right), & U_{n}(a) & :=T(a) B_{n}(a)^{-1}, \\
B_{n} & :=\left\{B_{n}(a)\right\}_{a \in A}, & U_{n} & :=\left\{U_{n}(a)\right\}_{a \in A},
\end{aligned}
$$

as before. $B_{n}$ and $U_{n}$ are measurable fields of operators, as measurable fields of operators remain measurable under inversion and addition. Let $f=\{f(a)\}_{a \in A}$ and $g=\{g(a)\}_{a \in A}$ be two measurable fields of vectors. Since, for all $a \in A, B_{n}(a)$ and $U_{n}(a)$ converge weakly to $|T(a)|$ and $U(a)$, respectively, it follows that

$$
a \mapsto\left\langle f(a), B_{n}(a) g(a)\right\rangle_{a}, \quad a \mapsto\left\langle f(a), U_{n}(a) g(a)\right\rangle_{a}
$$

converges almost everywhere to

$$
a \mapsto\langle f(a),|T(a)| g(a)\rangle_{a}, \quad \quad a \mapsto\langle f(a), U(a) g(a)\rangle_{a},
$$

respectively, and, therefore, the latter are measurable for all measurable fields of operators $f, g$. In consequence, $|T|=\{|T(a)|\}_{a \in A}$ and $U=\{U(a)\}_{a \in A}$ are measurable fields of operators. 


\section{APPENDIX B}

\section{Fourier transformation of tempered distributions}

Lemma B.1. Let $u \in \mathcal{S}^{\prime}\left(\mathbb{R}^{n}\right)$ be a tempered distribution.

- Suppose there exists a function $f \in L^{2}\left(\mathbb{R}^{n}\right)$ such that

$$
u(\varphi)=\int_{\mathbb{R}^{n}} f(x) \varphi(x) \mathrm{d} x \quad \forall \varphi \in \mathcal{S}\left(\mathbb{R}^{n}\right)
$$

Then $f$ is uniquely defined by this relation. In particular, we can identify $u$ with $f$ and write $u \in L^{2}\left(\mathbb{R}^{n}\right)$.

- $u \in L^{2}\left(\mathbb{R}^{n}\right)$ if and only if $\hat{u} \in L^{2}\left(\mathbb{R}^{n}\right)$. In that case, $\hat{u}$ is the Plancherel transform of $u$.

Proof.

- If there exists a function $f \in L^{2}\left(\mathbb{R}^{n}\right)$ such that

$$
u(\varphi)=\int_{\mathbb{R}^{n}} f(x) \varphi(x) \mathrm{d} x \quad \forall \varphi \in \mathcal{S}\left(\mathbb{R}^{n}\right)
$$

then $u$ satisfies

$$
|u(\varphi)| \leq\|f\|_{2} \cdot\|\varphi\|_{2}
$$

where $\|\cdot\|_{2}$ denotes the $L^{2}$-norm. Since $\mathcal{S}\left(\mathbb{R}^{n}\right)$ is dense in $L^{2}\left(\mathbb{R}^{n}\right)$ there exists a unique continuous extension $\bar{u}$ of $u$ to $L^{2}\left(\mathbb{R}^{n}\right)$ which by Riesz Representation theorem uniquely corresponds to a function $g \in L^{2}\left(\mathbb{R}^{n}\right)$ such that

$$
\bar{u}(\varphi)=\langle g, \varphi\rangle_{2} \quad \varphi \in L^{2}\left(\mathbb{R}^{n}\right) .
$$

By uniqueness $g$ must be equal to the complex conjugate of $f$.

- Suppose that $u=f \in L^{2}\left(\mathbb{R}^{n}\right)$. If $\hat{f}$ is the Plancherel transform of $f$ then we have

$$
\hat{u}(\varphi)=u(\hat{\varphi})=\int_{\mathbb{R}^{n}} f(x) \hat{\varphi}(x) \mathrm{d} x=\int_{\mathbb{R}^{n}} \hat{f}(x) \varphi(x) \mathrm{d} x,
$$

where the first equality is the definition of the Fourier transform of a distribution and the last equality follows from the Plancherel Theorem. It follows that $\hat{u} \in$ 
$L^{2}\left(\mathbb{R}^{n}\right)$ and $\hat{u}=\hat{f}$.

Conversely, if $\hat{u}=\hat{f} \in L^{2}\left(\mathbb{R}^{n}\right)$ then it follows analogously by inverse Fourier or Plancherel transformation that $u \in L^{2}\left(\mathbb{R}^{n}\right)$ and $u=f$.

Lemma B.2. Let $\mu$ be a finite measure of $\mathbb{R}^{n}$ and let $u_{\mu}$ be the tempered distribution given by

$$
u_{\mu}(\varphi)=\int_{\mathbb{R}^{n}} \varphi(x) \mathrm{d} \mu(x), \forall \varphi \in \mathcal{S}\left(\mathbb{R}^{n}\right)
$$

If $u_{\mu} \in L^{2}\left(\mathbb{R}^{n}\right)$ then $\mu$ is absolutely continuous with respect to the Lebesgue measure of $\mathbb{R}^{n}$.

Proof. Let $u_{\mu}=f \in L^{2}\left(\mathbb{R}^{n}\right)$ and let $u_{f}$ be the tempered distribution given by

$$
u_{f}(\varphi)=\int_{\mathbb{R}^{n}} f(x) \varphi(x) \mathrm{d} x, \forall \varphi \in \mathcal{S}\left(\mathbb{R}^{n}\right)
$$

Then, by definition $u_{\mu}=u_{f}$ and by Riesz-Markov-Kakutani Representation Theorem (cf. Thm. 2.9)

$$
\mathrm{d} \mu(x)=f(x) \mathrm{d} x .
$$

Hence, $\mu$ is absolutely continuous with respect to the Lebesgue measure. 


\section{Bibliography}

[1] G. S. Alberti, S. Dahlke, F. De Mari, E. De Vito, and H. Führ. Recent progress in shearlet theory: systematic construction of shearlet dilation groups, characterization of wavefront sets, and new embeddings. In Frames and other bases in abstract and function spaces, Appl. Numer. Harmon. Anal., pages 127-160. Birkhäuser/Springer, Cham, 2017.

[2] D.V. Alekseevsky and H. Baum. Recent Developments in Pseudo-Riemannian Geometry. ESI lectures in mathematics and physics. European Mathematical Society, 2008.

[3] S. T. Ali, J.-P. ANTOINE, and J. P. Gazeau. Coherent States, Wavelets and Their Generalizations. Graduate Texts in Contemporary Physics. Springer New York, 1999.

[4] P. Aniello, G. Cassinelli, E. de Vito, and A. Levrero. Square-integrability of induced representations of semidirect products. Reviews in Mathematical Physics, 10(03):301-313, April 1998.

[5] J.-P. Antoine and P. Vandergheynst. Wavelets on the n-sphere and related manifolds. Journal of Mathematical Physics, 39(8):3987-4008, August 1998.

[6] J.-P. Antoine and P. Vandergheynst. Wavelets on the 2-sphere: A group-theoretical approach. Applied and Computational Harmonic Analysis, 7(3):262-291, November 1999 .

[7] E. W. Aslaksen and J. R. Klauder. Continuous representation theory using the affine group. J. Mathematical Phys., 10:2267-2275, 1969.

[8] L. Auslander and B. Kostant. Polarization and unitary representations of solvable lie groups. Inventiones Mathematicae, 14(4):255-354, dec 1971.

[9] L. Auslander and C. C. Moore. Unitary representations of solvable Lie groups. Mem. Amer. Math. Soc., 62:199, 1966.

[10] L. Baggett. Representations of the Mautner group. I. Pacific J. Math., 77(1):7-22, 1978. 
[11] G. Blanchet and M. Charbit. Digital Signal and Image Processing Using Matlab®. John Wiley \& Sons, Inc., February 2015.

[12] N. Bourbaki. Éléments de mathématique. Première partie. (Fascicule III.) Livre III; Topologie générale. Chap. 3: Groupes topologiques. Chap. 4: Nombres réels. Troisième édition revue et augmentée. Actualités Sci. Indust., No. 1143. Hermann, Paris, 1960.

[13] N. Bourbaki. General topology. Chapters 1-4. Elements of Mathematics (Berlin). Springer-Verlag, Berlin, 1989. Translated from the French, Reprint of the 1966 edition.

[14] N. Bourbaki. General topology. Chapters 5-10. Elements of Mathematics (Berlin). Springer-Verlag, Berlin, 1989. Translated from the French, Reprint of the 1966 edition.

[15] N. Bourbaki. Integration. I. Chapters 1-6. Elements of Mathematics (Berlin). Springer-Verlag, Berlin, 2004. Translated from the 1959, 1965 and 1967 French originals by Sterling K. Berberian.

[16] N. Bourbaki. Integration. II. Chapters 7-9. Elements of Mathematics (Berlin). Springer-Verlag, Berlin, 2004. Translated from the 1963 and 1969 French originals by Sterling K. Berberian.

[17] I. D. Brown. Dual topology of a nilpotent Lie group. Ann. Sci. École Norm. Sup. (4), 6:407-411, 1973.

[18] B. Cahen. Some remarks on the notion of contraction of Lie group representations. Math. Morav., 14(1):35-46, 2010.

[19] A.-P. Calderón. Intermediate spaces and interpolation, the complex method. Studia Math., 24:113-190, 1964.

[20] S. De Bièvre. Coherent states over symplectic homogeneous spaces. Journal of Mathematical Physics, 30(7):1401-1407, 1989.

[21] J. Dixmier. $C^{*}$-algebras. North-Holland Publishing Co., Amsterdam-New YorkOxford, 1977. Translated from the French by Francis Jellett, North-Holland Mathematical Library, Vol. 15.

[22] J. Dixmier. von Neumann algebras, volume 27 of North-Holland Mathematical Library. North-Holland Publishing Co., Amsterdam-New York, 1981. With a preface by E. C. Lance, Translated from the second French edition by F. Jellett.

[23] A. H. Dooley. Contractions of Lie groups and applications to analysis. In Topics in modern harmonic analysis, Vol. I, II (Turin/Milan, 1982), pages 483-515. Ist. Naz. Alta Mat. Francesco Severi, Rome, 1983. 
[24] A. H. Dooley and J. W. Rice. Contractions of rotation groups and their representations. Math. Proc. Cambridge Philos. Soc., 94(3):509-517, 1983.

[25] M. Duflo and C. C. Moore. On the regular representation of a nonunimodular locally compact group. J. Functional Analysis, 21(2):209-243, 1976.

[26] S. Ebert and J. Wirth. Diffusive wavelets on groups and homogeneous spaces. Proc. Roy. Soc. Edinburgh Sect. A, 141(3):497-520, 2011.

[27] J. Elstrodt. Maß- und Integrationstheorie. Springer Berlin Heidelberg, 2018.

[28] P. Eymard. L'algèbre de Fourier d'un groupe localement compact. Bull. Soc. Math. France, 92:181-236, 1964.

[29] J. Ferrand. The action of conformal transformations on a riemannian manifold. Mathematische Annalen, 304(2):277-292, 1996.

[30] G. B. Folland. A Course in Abstract Harmonic Analysis. Studies in Advanced Mathematics. Taylor \& Francis, 1994.

[31] G. B. Folland. A course in abstract harmonic analysis. Textbooks in Mathematics. CRC Press, Boca Raton, FL, second edition, 2016.

[32] G. F. Frobenius. Über relationen zwischen den charakteren einer gruppe und denen ihrer untergruppen. Sitzungsberichte der Königlich Preussischen Akademie der Wissenschaften zu Berlin, pages 501-515, 1898.

[33] H. Führ. Abstract harmonic analysis of continuous wavelet transforms, volume 1863 of Lecture Notes in Mathematics. Springer-Verlag, Berlin, 2005.

[34] H. Führ. Generalized Calderón conditions and regular orbit spaces. Colloq. Math., 120(1):103-126, 2010.

[35] H. Führ. Continuous diffusion wavelet transforms and scale space over Euclidean spaces and noncommutative Lie groups. In Mathematical methods for signal and image analysis and representation, volume 41 of Comput. Imaging Vision, pages 123-136. Springer, London, 2012.

[36] D. Gabor. Theory of communication. Institution of Electrical Engineering, 1946.

[37] R. J. Glauber. Coherent and incoherent states of the radiation field. Phys. Rev. (2), 131:2766-2788, 1963.

[38] P. Goupillaud, A. Grossmann, and J. Morlet. Cycle-octave and related transforms in seismic signal analysis. Geoexploration, 23(1):85-102, October 1984.

[39] A. Grossmann, J. Morlet, and T. Paul. Transforms associated to square integrable group representations. I. General results. J. Math. Phys., 26(10):2473-2479, 1985. 
[40] M. Holschneider. Continuous wavelet transforms on the sphere. Journal of Mathematical Physics, 37(8):4156-4165, August 1996.

[41] A. Hulanicki and T. Pytlik. Cyclic vectors of induced representations. Proc. Amer. Math. Soc., 31:633-634, 1972.

[42] E. Inonu and E. P. Wigner. On the contraction of groups and their representations. Proceedings of the National Academy of Sciences, 39(6):510-524, 1953.

[43] O. Kallenberg. Random Measures, Theory and Applications. Springer International Publishing, 2017.

[44] E. Kaniuth and K.F. Taylor. Induced Representations of Locally Compact Groups. Cambridge Tracts in Mathematics. Cambridge University Press, 2013.

[45] A. A. Kirillov. Elements of the Theory of Representations (Grundlehren der mathematischen Wissenschaften). Springer, 1976.

[46] A. A. Kirillov. Lectures on the Orbit Method. Graduate studies in mathematics. American Mathematical Society, 2004.

[47] C. Kuratowski. Topologie. I. Espaces Métrisables, Espaces Complets. Monografie Matematyczne, vol. 20. Warszawa-Wrocław, 1948. 2d ed.

[48] G. Kutyniok and D. Labate. Shearlets: Multiscale Analysis for Multivariate Data. Applied and Numerical Harmonic Analysis. Birkhäuser Boston, 2012.

[49] R. L. Lipsman and J. Rosenberg. The behavior of Fourier transforms for nilpotent Lie groups. Trans. Amer. Math. Soc., 348(3):1031-1050, 1996.

[50] G. W. Mackey. Induced representations of locally compact groups. I. Ann. of Math. (2), 55:101-139, 1952.

[51] G. W. Mackey. Borel structure in groups and their duals. Trans. Amer. Math. Soc., 85:134-165, 1957.

[52] G. W. Mackey. Infinite-dimensional group representations. Bull. Amer. Math. Soc., 69:628-686, 1963.

[53] H. Moscovici. Coherent state representations of nilpotent Lie groups. Comm. Math. Phys., 54(1):63-68, 1977.

[54] R. Murenzi. Wavelet transforms associated to the n-dimensional euclidean group with dilations: Signal in more than one dimension. In Jean-Michel Combes, Alexander Grossmann, and Philippe Tchamitchian, editors, Wavelets, pages 239246, Berlin, Heidelberg, 1990. Springer Berlin Heidelberg. 
[55] A. Perelomov. Generalized Coherent States and Their Applications. Texts and Monographs in Physics. Springer-Verlag Berlin Heidelberg, 1 edition, 1986.

[56] W. Rudin. Reelle und komplexe Analysis. Oldenbourg, 1999.

[57] I. E. Segal. A class of operator algebras which are determined by groups. Duke Math. J., 18:221-265, 1951.

[58] N. Tatsuuma. Plancherel formula for non-unimodular locally compact groups. $J$. Math. Kyoto Univ., 12:179-261, 1972.

[59] M. W. Wong. Wavelet transforms and localization operators, volume 136 of Operator Theory: Advances and Applications. Birkhäuser Verlag, Basel, 2002. 


\section{Acknowledgments}

First of all, I want to thank my supervisor Prof. Dorothea Bahns for giving me the opportunity to work on the topic of my thesis. During the last years, I learned a lot and developed myself further. This is mainly due to her support and guidance. Moreover, I'm grateful to my second supervisor Prof. Thomas Schick, who also provided me with valuable advice and support, and to the secretaries of the Mathematical Institute for the practical help.

Furthermore, I want to thank my colleagues at the Mathematical Institute for the great time. All above, Fabian Waibel and Rebecca Möser, who were not only my office colleagues but became very dear friends. Moreover, I'm grateful to Christoff Krüger for valuable discussions on questions related to differential geometry.

Last but not least, I want to thank my parents, Steffen and Sabine Blobel, for the emotional support. The last years have not always been easy. But my parents were always there for me and did their best to motivate and support me. Likewise, I want to thank my friends, Merle Behr, Markus Schmitt, Tobias Binder, Thomas Kotzott, Bodo Schwabe, and Fabian Waibel, and my brother, Falk Blobel, for the unforgettable and amazing time we spent together in Göttingen. 


\section{Publications}

- T. Binder, B. Blobel, J. Harz, and K. Mukaida. Dark Matter bound-state formation at higher order: a non-equilibrium quantum field theory approach. Journal of High Energy Physics, 2020(9), September 2020 


\section{Erklärung}

1. Hiermit erkläre ich, dass diese Arbeit weder in gleicher noch in ähnlicher Form bereits anderen Prüfungsbehören vorgelegen hat.

Weiter erkläre ich, dass ich mich an keiner anderen Hochschule um einen Doktorgrad beworben habe.

Göttingen, den

(Unterschrift)

2. Hiermit erkläre ich eidesstattlich, dass diese Dissertation selbständig und ohne unerlaubte Hilfe angefertigt wurde.

Göttingen, den

(Unterschrift) 